\title{
Mass, linear momentum and kinetic energy of bipolar flows in protoplanetary nebulae
}

\author{
V. Bujarrabal ${ }^{1}$, A. Castro-Carrizo ${ }^{1}$, J. Alcolea ${ }^{1}$, and C. Sánchez Contreras ${ }^{1,2}$ \\ 1 Observatorio Astronómico Nacional, Apartado 1143, 28800 Alcalá de Henares, Spain \\ e-mail: bujarrabal,j.alcolea, carrizo@oan.es \\ 2 Jet Propulsion Laboratory, 4800 Oak Grove Drive, Pasadena, CA 91109, USA \\ e-mail: sanchez@eclipse.jpl.nasa.gov
}

Received 16 February 2001 / Accepted 26 July 2001

\begin{abstract}
We have studied the CO emission from protoplanetary nebulae (PPNe). Our sample is composed of 37 objects and includes, we think, all well identified PPNe detected in CO, together with the two yellow hypergiants emitting in $\mathrm{CO}$ and one young PN. We present a summary of the existing CO data, including accurate new observations of the ${ }^{12} \mathrm{CO}$ and ${ }^{13} \mathrm{CO} J=1-0$ and $J=2-1$ lines in 16 objects. We identify in the nebulae a slowly expanding shell (represented in the spectra by a central core) and a fast outflow (corresponding to the line wings), that in the well studied PPNe is known to be bipolar. Excluding poor data, we end up with a sample of 32 sources (including the 16 observed by us); fast flows are detected in 28 of these nebulae, being absent in only 4 . We present a method to estimate from these data the mass, "scalar" momentum and kinetic energy of the different components of the molecular outflows. We argue that the uncertainties of our method can hardly lead to significant overestimates of these parameters, although underestimates may be present in not well studied objects. The total nebular mass is often as high as $\sim 1 M_{\odot}$, and the mass-loss rate, that (presumably during the last stages of the AGB phase) originated the nebula, had typical values $\sim 10^{-4} M_{\odot} \mathrm{yr}^{-1}$. The momentum corresponding to this mass ejection process in most studied nebulae is accurately coincident with the maximum momentum that radiation pressure, acting through absorption by dust grains, is able to supply (under expected conditions). We estimate that this high-efficiency process lasts about 1000-10000 yr, after which the star has ejected a good fraction of its mass and the AGB phase ends. On the other hand, the fast molecular outflows, that have probably been accelerated by shock interaction with axial post-AGB jets, carry a significant fraction of the nebular mass, with a very high momentum (in most cases between $10^{37}$ and $10^{40} \mathrm{~g} \mathrm{~cm} \mathrm{~s}^{-1}$ ) and very high kinetic energy (usually between $10^{44}$ and $10^{47} \mathrm{erg}$ ). In general, yellow hypergiants and post-AGB objects with low initial mass show nebular masses and momenta that are, respectively, higher and lower than these values. We compare the momenta of the fast outflows with those that can be supplied by radiation pressure, taking into account the expected short acceleration times and some effects that can increase the momentum transfer. We find that in about $80 \%$ of PPNe, the fast molecular flows have too high momenta to be powered by radiation pressure. In some cases the momentum of the outflow is $~ 1000$ larger than that carried by radiation pressure; such high factors are difficult to explain even under exceptional conditions. Wind interaction is the basic phenomenon in the PN shaping from the former AGB envelopes; we conclude that this interaction systematically takes place along a dominant direction and that this process is not powered by radiation pressure. Due to the lack of theoretical studies, the possible momentum source remains a matter of speculation.
\end{abstract}

Key words. stars: AGB and post-AGB - stars: circumstellar matter - radio-lines: stars - planetary nebulae

\section{Introduction}

Recent observations of molecular lines in the protoplanetary nebulae (PPNe) $\mathrm{OH} 231.8+4.2$ (Sánchez Contreras et al. 1997; Alcolea et al. 2001), M 1-92 (Bujarrabal et al. 1998a) and HD 101584 (Olofsson \& Nyman 1999) have pointed out the presence of very massive and fast bipolar

Send offprint requests to: V. Bujarrabal, e-mail: bujarrabal@oan.es outflows. The dynamics of these well studied objects is dominated by a fast flow in the direction of the symmetry axis of the nebula, with velocities $\sim 100 \mathrm{~km} \mathrm{~s}^{-1}$. Values of the total nebular mass close to $1 M_{\odot}$ are found. A significant fraction of the mass belongs to the fast component, that therefore carries very high kinetic momentum and energy, $\sim 3 \times 10^{39} \mathrm{~g} \mathrm{~cm} \mathrm{~s}^{-1}$ and $10^{46} \mathrm{erg}$, respectively. (A first measurement of the high mass and momentum in OH 231.8+4.2 is due to Knapp 1986, who also compared 
the momenta carried by the nebular mass and the stellar light.) Typically, the gas in these outflows shows a very low temperature, $\sim 10-20 \mathrm{~K}$, this is the reason why they are mainly detected by means of observations of molecular lines (hot nebular gas is also detected, from optical and NIR lines for instance, but its mass is much smaller). It is thought that these axial flows are the result of the acceleration of the previous AGB wind, that is massive but slow, by shock interaction with the post-AGB ejections, that would be much faster and run along the axial direction. Observations of dust emission and scattered light also allow the detection of the massive component of the nebula, confirming the mass and density values found from molecular lines, but do not provide information on the kinematics, see e.g. Bujarrabal et al. (1997, 1998b), Sahai et al. (1999b).

Even if the studied objects in those papers are quite luminous (typically, $L \sim 10^{4} L_{\odot}$ ), the linear momentum associated to the fast molecular flow is too high (by orders of magnitude) to be supplied by radiation pressure, given the relatively short times during which the windinteraction phenomenon probably took place, 100-200 yr. In this case, we will say that the mass outflow is "overluminous". For example, in M 1-92, the momentum carried by the molecular outflow is $\sim 3 \times 10^{39} \mathrm{~g} \mathrm{~cm} \mathrm{~s}^{-1}$; the star emits about $4 \times 10^{37} \mathrm{erg} \mathrm{s}^{-1}$, equivalent in momentum to $4 \times 10^{34} \mathrm{~g} \mathrm{~cm} \mathrm{~s}^{-1}$ per year, so the star would need about $10^{5}$ yr to release such a high linear momentum, but the wind interaction lasted just $\sim 100 \mathrm{yr}$ (with an upper limit of $900 \mathrm{yr}$, the total post-AGB life of the object; see more details in Bujarrabal et al. 1998a).

The measured kinetic energies and momenta are so high that it is difficult to find other mechanisms that could power these very energetic ejections satisfying the principles of conservation. (Similar "overluminous" flows are well known to occur in star-forming regions, for which the accretion of material from the protostellar cloud is invoked to power the bipolar jets.) If such a property is found to be typical in PPNe, we would have to conclude that, from the point of view of the dynamics involved, presently we have no explanation for the shaping of planetary nebulae from the previous AGB envelopes. However, only a few nebulae have been properly studied at this respect. In this paper we present a systematic study of the molecular outflows in PPNe. We have cataloged all well identified PPNe showing $\mathrm{CO}$ emission and have performed accurate new observations in a sizable number of objects. From the $\mathrm{CO}$ data, we have calculated the nebular mass and the linear momentum and kinetic energy of the flows. Our results are compared with the momentum and kinetic energy that can be provided by the stellar radiation pressure.

\section{Source sample}

The goal of this work is to estimate the mass of the bipolar outflows in PPNe, as well as the momentum and energy carried by them. We used for that purpose CO emission, since, as far as we know, it is the best tracer of the total nebular mass. We have chosen a sample of PPNe emitting in $\mathrm{CO}$; we think that our sample includes all well identified PPNe that have been detected in $\mathrm{CO}$ emission (up to December 2000). We also included three related objects (see below). The basic criterion for the classification of a nebula as protoplanetary is the presence of a cool and thick circumstellar envelope and a bimodal spectral energy distribution (SED), showing that the envelope is well detached from the central star and that the stellar temperature is between $\sim 3000$ and $\sim 30000 \mathrm{~K}$ (in general corresponding to intermediate spectral types: WC10-11, B, A, F, G, or K; in many cases, independent studies have confirmed the stellar type). Therefore, the selected objects lost large amounts of mass in the past, when the central stars were probably cooler. These central stars seem to be now evolving toward very hot objects, and will soon illuminate and ionize the nebula. Objects associated to interstellar clouds are rejected. Standard PNe showing strong $\mathrm{CO}$ emission, and sometimes wide line wings (as for instance M 1-16), were not considered in our study. For further discussion on these criteria, see e.g. Kwok (1993).

We did not include nebulae of uncertain classification. Accordingly, we have not studied objects like AFGL 190 (IRAS 01144+6658), IRAS 19454+2920 and IRAS $19480+2504$, that show a heavy envelope (detected by means IRAS photometry and CO line emission), because the temperature of their central stars is not known (see Volk \& Kwok 1989; Likkel et al. 1991; Volk et al. 1993; Groenewegen et al. 1998). However, we have included in our sample IRAS $23321+6545$, IRAS 20028+3910, and IRAS $22574+6609$, even if the spectral types of the stars are unknown, because they show a clearly bimodal SED, indicating the emissions of a cool and massive envelope and probably of a relatively hot stellar component, as well as a bipolar image in HST high-resolution observations (Ueta et al. 2000). IRAS 22574+6609, moreover, shows FIR dust features usually associated to PPNe (Hrivnak \& Kwok 1991). OH231.8+4.2 is assumed to be a PPN, in spite of the late (and peculiar) spectrum of its central star, because it presents a massive envelope with well developed shocks, a property characteristic of post-AGB objects and very rare in AGB stars. CRL 2477 is assumed to be a PPN because of its bimodal SED (Groenewegen et al. 1996), but the nature of the central star is controversial and it is possible that two evolved objects are coincident in the line of sight.

NGC 7027 is not a PPNe but a young PN; it is included in our list, in spite of its very hot central star, because its envelope has a short kinematical age ( $1000 \mathrm{yr}$, Graham et al. 1993) and a massive molecular component, similarly to PPNe. Finally, we have included in our list two objects that show heavy envelopes and are thought to be hypergiants, IRC +10420 and AFGL 2343 (the only two hypergiants surrounded by massive nebulae, see e.g. de Jager 1998 and discussion in 5.1.7). The evolution of such objects is uncertain, but it seems that they were cooler in the past, when they ejected most of the circumstellar shell, and that they are becoming hotter rapidly. 
Table 1. Sample of PPNe observed in CO by us.

\begin{tabular}{|c|c|c|c|c|c|c|c|c|}
\hline name & $\begin{array}{c}\text { observed c } \\
\alpha(2000)\end{array}$ & $\begin{array}{r}\text { oordinates } \\
\delta(2000) \\
\end{array}$ & $\begin{array}{l}V_{\text {sys }} \text { LSR } \\
\left(\mathrm{km} \mathrm{s}^{-1}\right) \\
\end{array}$ & $\begin{array}{l}\text { spectral } \\
\text { type }\end{array}$ & $\begin{array}{c}i \\
\left({ }^{\circ}\right)\end{array}$ & $\begin{array}{c}D \\
(\mathrm{kpc}) \\
\end{array}$ & $\begin{array}{c}L \\
\left(10^{3} L_{\odot}\right) \\
\end{array}$ & comments \\
\hline IRAS $04296+3429$ & 043257.0 & 343613 & -65 & $6500(\mathrm{~F} 5)^{1,2}$ & $25^{3}$ & $5 ?^{4,2}$ & $7^{2}$ & \\
\hline CRL 618 & 044253.4 & 360654 & -21 & $\mathrm{~B} 0^{\mathrm{s}}$ & $45^{5}$ & $1.7^{6}$ & $30^{7}$ & \\
\hline $\begin{array}{l}\text { Frosty Leo } \\
\quad \text { IRAS } 09371+1\end{array}$ & $\begin{array}{r}601 \\
093954.0 \\
212\end{array}$ & 115854 & -12 & $\mathrm{~K} 7 \mathrm{III}{ }^{8}$ & $15^{9}$ & $3^{8}$ & $2.7^{10}$ & \\
\hline $\begin{array}{c}\text { IRAS } 17436+5003 \\
\text { HD } 161796\end{array}$ & 174455.5 & 500240 & -35 & $\mathrm{~F} 2-5 \mathrm{Ib}^{11}$ & $?$ & $4^{7}$ & $60^{7}$ & \\
\hline $\begin{array}{l}\text { He } 3-1475 \\
\quad \text { IRAS } 17423-17\end{array}$ & $\begin{array}{l}174514.1 \\
55\end{array}$ & -175647 & 48 & $\mathrm{Be}^{12,13}$ & $60^{13}$ & $5^{13}$ & $9^{13}$ & \\
\hline $\begin{array}{l}89 \text { Her } \\
\quad \text { IRAS } 17534+2\end{array}$ & $\begin{array}{l}175525.1 \\
603\end{array}$ & 260259 & -8 & F2Ibe ${ }^{\mathrm{s}}$ & ISOT. ${ }^{0}$ & $0.6^{14}$ & $3.3^{14}$ & high-latitude yellow giant \\
\hline $\begin{array}{l}\text { AFGL } 2343 \\
\quad \text { IRAS } 19114+00\end{array}$ & $\begin{array}{l}191358.6 \\
02\end{array}$ & 000732 & 98 & G5Ia $^{11}$ & ISOT. $^{0}$ & $5.6^{\mathrm{H}}$ & $580^{15}$ & yellow hypergiant \\
\hline $\begin{array}{l}\text { IRC }+10420 \\
\quad \text { IRAS } 19244+1\end{array}$ & $\begin{array}{l}192648.0 \\
115\end{array}$ & 112117 & 76 & $\mathrm{~F} 8 \mathrm{Ia}^{16}$ & ISOT. $^{0}$ & $5^{16}$ & $700^{16}$ & yellow hypergiant \\
\hline $\begin{array}{c}\text { IRAS } 19500-1709 \\
\text { SAO } 163075\end{array}$ & 195252.6 & -170150 & 25 & $\mathrm{~F} 2-6^{11}$ & $?$ & $1^{7}$ & $1.5^{7}$ & \\
\hline $\begin{array}{l}\text { CRL } 2477 \\
\quad \text { IRAS } 19548+3\end{array}$ & $\begin{array}{l}195648.4 \\
035\end{array}$ & 304400 & 5 & $?^{17}$ & $?$ & $1.3^{17}$ & $4^{17}$ & PPN? \\
\hline $\begin{array}{l}\text { CRL } 2688 \\
\quad \text { Egg Nebula, }\end{array}$ & $\begin{array}{c}210218.8 \\
1003+3629)\end{array}$ & 364138 & -35 & F5 Iae ${ }^{\mathrm{s}}$ & $15^{18,19}$ & $1.2^{19}$ & $25^{19,6}$ & \\
\hline $\begin{array}{l}\text { NGC } 7027 \\
\quad(21052+4202)\end{array}$ & 210701.6 & 421410 & 26 & pec. & $30^{20}$ & $1^{21}$ & $10^{21}$ & young PN \\
\hline $\begin{array}{c}\text { IRAS } 22272+5435 \\
\text { HD } 235858\end{array}$ & 222910.4 & 545107 & -28 & $\mathrm{G} \mathrm{Ia}^{22}$ & $30^{4}$ & $1.7^{23}$ & $8.3^{23}$ & \\
\hline IRAS $23304+6147$ & 233245.0 & 620349 & -16 & $\mathrm{G} 2 \mathrm{Ia}^{22}$ & $90 ?^{24}$ & $1.3^{25}$ & $1^{24}$ & \\
\hline IRAS $23321+6545$ & 233422.7 & 660151 & -55 & $?$ & $?$ & $?$ & $0.6^{26,1 \mathrm{kpc}}$ & \\
\hline $\begin{array}{l}\text { M } 2-56 \\
\quad \text { IRAS } 23541+7\end{array}$ & 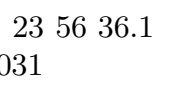 & 704817 & -27 & $\mathrm{Be}^{\mathrm{s}}$ & $15^{0}$ & $3^{27}$ & $10^{27}$ & \\
\hline
\end{tabular}

References: (s) Simbad database; (H) Hipparcos; (0) this paper; (1) Decin et al. (1998); (2) Klochkova et al. (1999); (3) Sahai (1999); (4) Meixner et al. (1997); (5) Neri et al. (1992); Yamamura et al. (1994); (6) Calvet \& Cohen (1978); (7) Bujarrabal et al. (1992); (8) Mauron et al. (1989); (9) Roddier et al. (1995); (10) Forveille et al. (1987); (11) Hrivnak et al. (1989); (12) Borkowski et al. (1997); (13) Riera et al. (1995); (14) Alcolea \& Bujarrabal (1995); (15) Reddy \& Hrivnak (1999); (16) de Jager (1998), Jones et al. (1993); (17) Groenewegen et al. (1996); (18) Sahai et al. (1998); (19) Skinner et al. (1997); (20) Graham et al. (1993); (21) Jourdain de Muizon et al. (1990), Graham et al. (1993); (22) Hrivnak (1995); (23) Szczerba et al. (1997); (24) Hrivnak \& Kwok (1991); (25) Woodsworth et al. (1990); (26) Volk et al. (1993), luminosity for a distance of 1 kpc; (27) Goodrich (1991).

So these hypergiants are very probably following an evolutionary path similar to that of PPNe. In general, these objects are included in our list to allow a comparison of the properties of PPNe and of related objects.

In our sample we include the PPNe with low initial mass in which CO has been certainly detected: 89 Her, the Red Rectangle, M 2-9 and R Sct (see Alcolea \& Bujarrabal 1991; R Sct is a peculiar RV Tau variable). We note that PPNe with low initial mass show an anomalously low CO emission; in particular, $\mathrm{CO}$ is not detected in other RV Tau variables and in M 1-91. We recall that the post-redgiant evolution of these low-mass stars may be very slow and that they will perhaps not become standard PNe, due to the extreme dilution of the expanding shell.

We have performed high-sensitivity observations (Sect. 3) of some PPNe showing CO line emission, including in particular a well studied PPN that seems to have no wings in the CO lines, IRAS $22272+5435$. In general, we selected for observation objects for which useful ${ }^{13} \mathrm{CO} J=1-0$ data were expected, also trying to sample the variety of sources described above. For the objects not observed by us, we have taken data from the literature. The final sample is defined in Tables 1 and 2, respectively for the sources for which the relevant $\mathrm{CO}$ data have been 
Table 2. Sample of PPNe observed in CO in other papers.

\begin{tabular}{|c|c|c|c|c|c|c|c|c|c|}
\hline \multirow[t]{2}{*}{ name } & \multicolumn{2}{|c|}{ coordinates } & \multirow[t]{2}{*}{ ref. } & \multirow{2}{*}{$\begin{array}{l}V_{\text {sys }} \text { LSR } \\
\left(\mathrm{km} \mathrm{s}^{-1}\right)\end{array}$} & \multirow{2}{*}{$\begin{array}{l}\text { spectral } \\
\text { type }\end{array}$} & \multirow{2}{*}{$\begin{array}{c}i \\
\left({ }^{\circ}\right)\end{array}$} & \multirow{2}{*}{$\begin{array}{c}D \\
(\mathrm{kpc})\end{array}$} & \multirow{2}{*}{$\begin{array}{c}L \\
\left(10^{3} L_{\odot}\right)\end{array}$} & \multirow[t]{2}{*}{ comments } \\
\hline & $\alpha(2000)$ & $\delta(2000)$ & & & & & & & \\
\hline $\begin{array}{l}\text { Red Rectangle } \\
\text { IRAS 06176-10 }\end{array}$ & $\begin{array}{l}061958.2 \\
36\end{array}$ & -103815 & $\mathrm{H}$ & $0^{1}$ & $\mathrm{~A} 1^{2,3}$ & $15^{4,5}$ & $0.38^{\mathrm{H}}$ & $1^{6}$ & low-mass star \\
\hline $\begin{array}{l}\text { IRAS } 07134+1005 \\
\text { HD } 56126\end{array}$ & $\begin{array}{lll}07 & 16 & 10.3\end{array}$ & 095948 & $\mathrm{~s}$ & $72^{7}$ & $\mathrm{~F} 5^{8}$ & $50^{9,10}$ & $3^{7,9,10,11}$ & $13.5^{10}$ & \\
\hline $\begin{array}{l}\text { OH } 231.8+4.2 \\
\quad \text { IRAS } 07399-14\end{array}$ & 074216.9 & -144250 & 12 & $33^{13}$ & M9III ${ }^{13}$ & $40^{13}$ & $1.5^{13}$ & $10^{13}$ & well studied \\
\hline $\begin{array}{l}\text { Hen } 3-401 \\
\quad \text { IRAS } 10178-59\end{array}$ & 101932.5 & -601329 & 14 & $-30^{15}$ & $\mathrm{~B} 1^{15,16}$ & $15 ?^{14}$ & $3^{15}$ & $3.6^{14}$ & \\
\hline $\begin{array}{l}\text { Roberts } 22 \\
\quad \text { IRAS } 10197-57\end{array}$ & 102133.8 & -580548 & 17 & $0^{15}$ & $\mathrm{~A} 2 \mathrm{Iab}^{18}$ & $15^{17}$ & $2^{17}$ & $30^{17}$ & \\
\hline $\begin{array}{l}\text { HD } 101584 \\
\quad \text { IRAS } 11385-55\end{array}$ & 714058.8 & -553426 & $\mathrm{H}$ & $41^{19}$ & F0Iape ${ }^{\mathrm{s}}$ & $?$ & $1^{20}$ & $3^{20}$ & well studied \\
\hline $\begin{array}{l}\text { Boomerang Nebula } \\
\text { IRAS 12419-54 }\end{array}$ & 124445.5 & -543112 & 21 & $-4^{15}$ & G0III ${ }^{15}$ & $?$ & $1.5^{21}$ & $0.3^{21}$ & well studied \\
\hline $\begin{array}{l}\text { He } 2-113 \\
\quad \text { IRAS } 14562-54\end{array}$ & 6 & -541808 & 22 & $-56^{23}$ & $\mathrm{WC} 10^{24}$ & $?$ & $1.2^{24}$ & $5^{24}$ & \\
\hline $\begin{array}{l}\mathrm{Mz}-3 \\
\quad \text { IRAS } 16133-51\end{array}$ & 161713.6 & -515906 & 25 & $-17^{15}$ & $\mathrm{~B} 0^{26}$ & $20^{27}$ & $1.8^{15,28}$ & $5.7^{25,26}$ & \\
\hline $\begin{array}{l}\text { M } 2-9 \\
\quad \text { IRAS } 17028-10\end{array}$ & 470537.9 & 100832 & 29 & $80^{29}$ & $\mathrm{Be}^{30}$ & $17^{29}$ & $0.64^{31}$ & $0.55^{31}$ & low-mass star \\
\hline $\begin{array}{l}\text { CPD }-568032 \\
\quad \text { IRAS } 17047-56\end{array}$ & 170900.9 & -565448 & $\mathrm{H}$ & $-60^{32,23}$ & $\mathrm{WC} 10^{24}$ & $?$ & $1.5^{24}$ & $5.2^{24}$ & \\
\hline $\begin{array}{c}\text { IRAS 17150-3224 } \\
\text { AFGL } 6815\end{array}$ & $1718 \quad 19.7$ & -322721 & 33 & $14^{34}$ & $\mathrm{G} 2^{\mathrm{s}}$ & $?$ & $2.42^{35}$ & $11^{35,36}$ & \\
\hline $\begin{array}{l}\text { OH } 17.7-2.0 \\
\text { IRAS } 18276-14\end{array}$ & 183030.7 & -142857 & 37 & $61^{38}$ & $\mathrm{~F} 0^{39,40}$ & $?$ & $2^{38}$ & $2.9^{38}$ & \\
\hline $\begin{array}{l}\text { R Sct } \\
\quad \text { IRAS } 18448-05\end{array}$ & 184729.0 & -054219 & $\mathrm{H}$ & $56^{41}$ & $\mathrm{G} 0-\mathrm{K} 2^{32}$ & $?$ & $0.4^{6}$ & $4^{6}$ & low-mass star \\
\hline $\begin{array}{l}\text { M } 1-92 \\
\quad \text { IRAS } 19343+2\end{array}$ & $\begin{array}{l}193618.9 \\
26\end{array}$ & 293250 & 43 & $-1^{43}$ & $\mathrm{~B} 0.5 \mathrm{IV}^{\mathrm{s}}$ & $35^{43}$ & $2.5^{43}$ & $10^{43}$ & well studied \\
\hline $\begin{array}{c}\text { IRAS } 19475+3119 \\
\text { HD } 331319\end{array}$ & 194929.6 & 312716 & $\mathrm{~s}$ & $18^{44}$ & F3Ia ${ }^{44}$ & $?$ & $6^{45}$ & $12.6^{0,45}$ & \\
\hline IRAS $20000+3239$ & 200159.4 & 324732 & 40 & $13.5^{44}$ & G8Ia ${ }^{8}$ & $?$ & $?$ & $0.55^{46,1 \mathrm{kpc}}$ & \\
\hline IRAS $20028+3910$ & 200435.9 & 391845 & 47 & $5.9^{44}$ & $?$ & $?$ & $2.5^{11,44,48}$ & $6.6^{11,35,44,48}$ & \\
\hline IRAS $21282+5050$ & 212958.5 & 510401 & 49 & $18^{50}$ & O9.5,WC11 ${ }^{51}$ & $90^{9}$ & $3^{9}$ & $5.3^{9}$ & \\
\hline IRAS $22223+4327$ & 222431.0 & 434309 & 40 & $-30^{48}$ & $\mathrm{GOIa}^{8}$ & $?$ & $?$ & $0.36^{46,1 \mathrm{kpc}}$ & \\
\hline IRAS $22574+6609$ & 225918.3 & 662547 & 33 & $-64^{44}$ & $?$ & $?$ & $?$ & $0.15^{52,1 \mathrm{kpc}}$ & \\
\hline
\end{tabular}

References : (s) Simbad database; (H) Hipparcos parallaxes; (0) this paper; (1) Jura et al. (1995); (2) Kelly \& Latter (1995); (3) Knapp et al. (1995); (4) Roddier et al. (1995); (5) López et al. (1995); (6) Alcolea \& Bujarrabal (1991); (7) Bujarrabal et al. (1992); (8) Hrivnak (1995); (9) Meixner et al (1997); (10) Dayal et al. (1998); (11) Yuasa et al. (1999); (12) Sánchez Contreras et al. (2000); (13) Sánchez Contreras et al. (1997) and Kastner et al. (1992); (14) Sahai et al. (1999a); (15) Bujarrabal \& Bachiller (1991); (16) Allen (1978); (17) Sahai et al. (1999b); (18) Allen et al. (1980); (19) Olofsson \& Nyman (1999); (20) Trams et al. (1990); (21) Sahai \& Nyman (1997); (22) Andrei et al. (1999) and Assafin et al. (1996); (23) Knapp et al. (1990); (24) De Marco \& Crowther (1998); (25) Van der Veen et al. (1989); (26) Cohen et al. (1978); (27) Meaburn \& Walsh (1985) and Redman et al. (2000); (28) Quinn et al. (1996); (29) Zweigle et al. (1997); (30) Calvet \& Cohen (1978) and Swings \& Andrillat (1979); (31) Schwartz et al. (1997); (32) Nyman et al. (1992); (33) Ueta et al. (2000); (34) Hu et al. (1993); (35) Loup et al. (1993); (36) Kwok et al. (1996); (37) Bowers et al. (1983); (38) Heske et al. (1990), van der Veen et al. (1995); (39) Le Bertre et al. (1989); (40) Meixner et al. (1999); (41) Bujarrabal et al. (1990); (42) Shenton et al. (1994); (43) Bujarrabal et al. (1997) and (1998a); (44) Likkel et al. (1991); (45) Likkel et al. (1987); (46) Kwok et al. (1995); (47) Neri et al. (1998); (48) Omont et al. (1993); (49) Meixner et al. (1998); (50) Likkel et al. (1988); (51) Crowther et al. (1998); (52) Hrivnak \& Kwok (1991). 


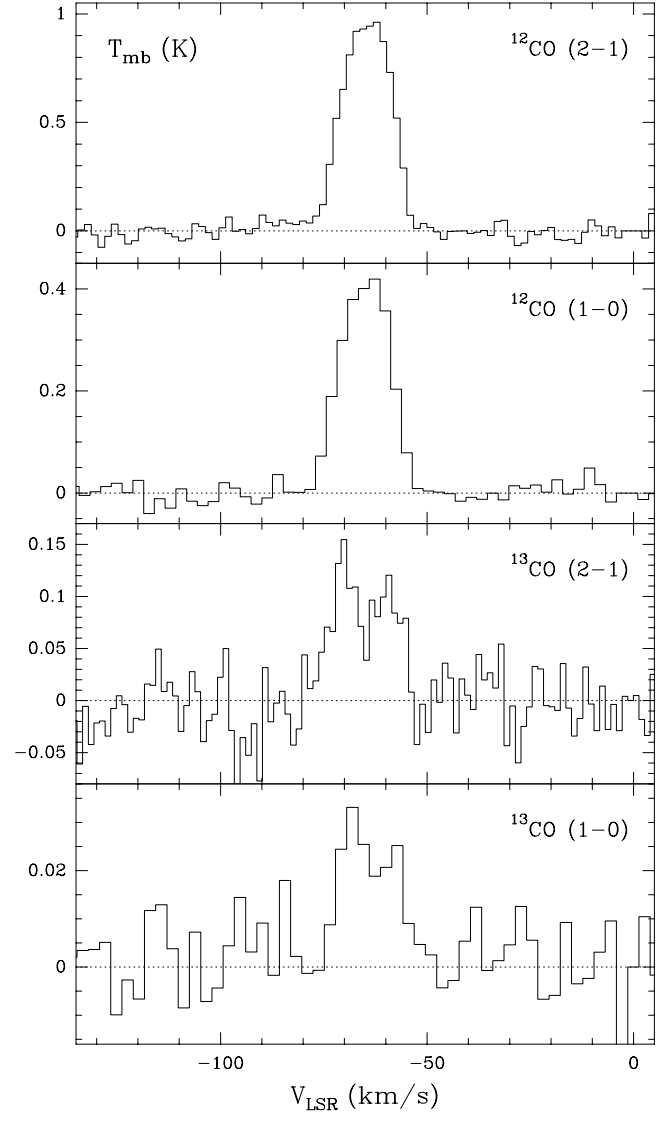

Fig. 1. CO observations of IRAS 04296+3429.

and have not been obtained in this work. In these tables we include the most usual source name and the IRAS catalog name, the coordinates, the adopted systemic velocity $\left(V_{\text {sys }}\right)$, the spectral type of the central star, the inclination of the nebula axis with respect to the plane of the sky $(i)$, the distance $(D)$ and the total luminosity calculated for this distance. Relevant references used to calculate the above parameters are given by the superindexes.

The coordinates given in Table 1 are those observed by us, in Table 2 we show the best coordinates we have found in the literature. The systemic velocity is deduced from the CO profiles. When the profiles are composite, with a central intense component and wide wings, we chose the centroid of the central component, since in well studied objects it seems to correspond to the part of the envelope not affected by the post-AGB wind interaction (e.g. Bujarrabal et al. 1998a, Sect. 8.1). When such a structure is not clear, we take for $V_{\text {sys }}$ the line centroid. The values for $i$ and $D$ are taken from specific studies on each nebula. When the inclination is not known, we will adopt the intermediate value $i=30^{\circ}$ (see discussion in Sect. 4). Note that the distance is sometimes poorly known in PPNe. Some comments on the sources, mostly related to their evolutionary status, are also given.

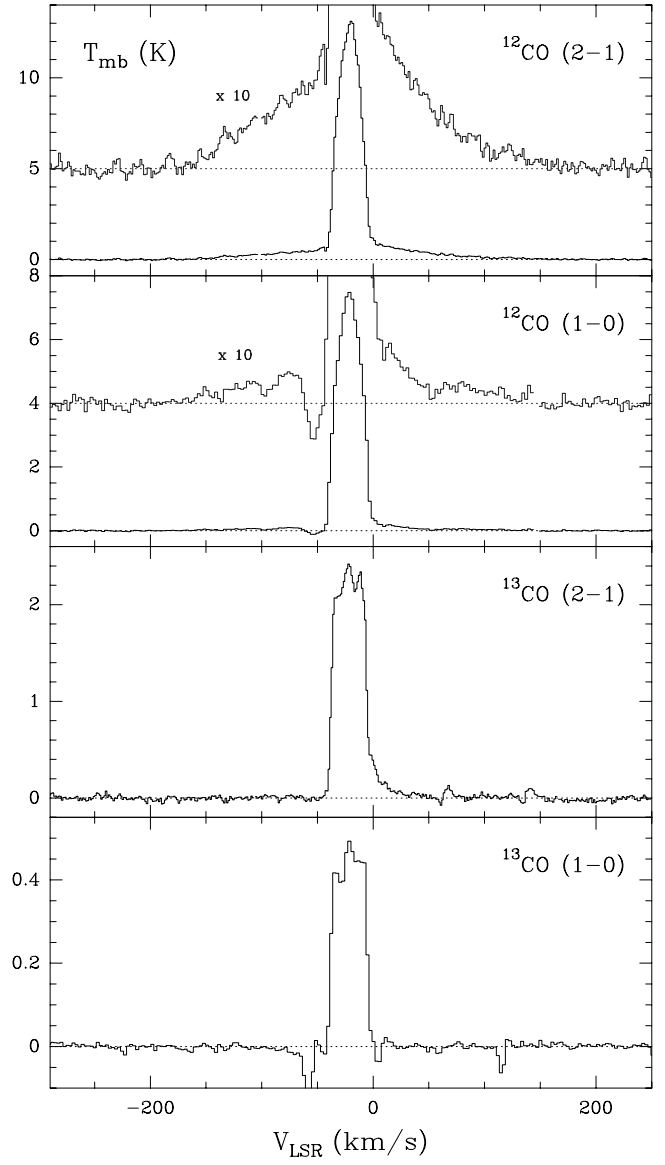

Fig. 2. CO observations of CRL 618.

\section{New CO observations}

We have performed observations of the $J=1-0$ and $J=2-1$ transitions of ${ }^{12} \mathrm{CO}$ and ${ }^{13} \mathrm{CO}$ in a sample of 16 protoplanetary nebulae (PPNe) and related objects, using the IRAM $30 \mathrm{~m}$ radiotelescope, at Pico de Veleta (Granada, Spain). Two observing runs were necessary, in August and November 1998. SIS receivers working in the 3 and $1 \mathrm{~mm}$ bands were used, often simultaneously. The receivers were tuned always in SSB mode, with typical system temperatures of $\sim 1000 \mathrm{~K}$, at $\lambda=1.3 \mathrm{~mm}$, and $500 \mathrm{~K}$, at $\lambda=2.6 \mathrm{~mm}$ (in units of $T_{\mathrm{mb}}$, see below). Spectral resolutions between 0.3 and $2.6 \mathrm{~km} \mathrm{~s}^{-1}$ were used.

Weather conditions were good for most observations, with zenith opacities at $230 \mathrm{GHz}$ ranging from about 0.3 to 0.8 . The pointing of the telescope was verified every about two hours or every time we moved into a new target, by observing continuum sources close in the sky to it. The spatial resolution is of $12-13^{\prime \prime}$ at $1.3 \mathrm{~mm}$ and of about $22^{\prime \prime}$ at $2.6 \mathrm{~mm}$.

The data presented here are calibrated in units of Main Beam Rayleigh-Jeans-equivalent Antenna Temperature, $T_{\mathrm{mb}}$, using the chopper-wheel method by observing hot (ambient) and cold loads (liquid nitrogen). In addition, observations of well known intense sources were used to check the calibration. In particular we took as standard the AGB star IRC+10216, for which we used the following 


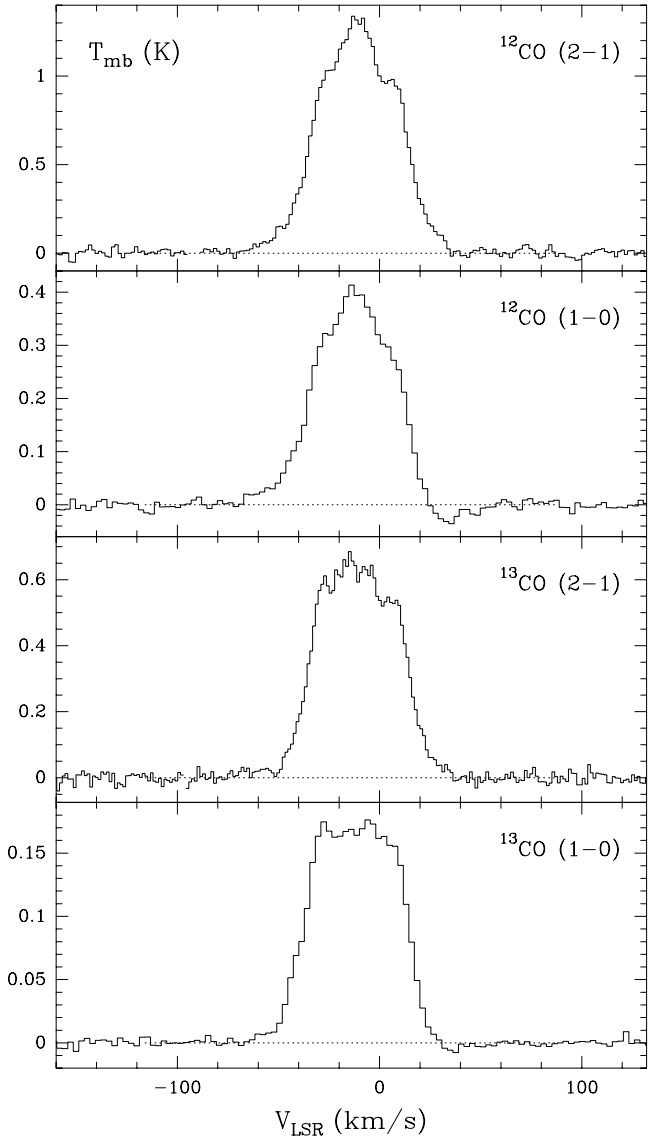

Fig. 3. CO observations of Frosty Leo.

intensities at the central velocity: $T_{\mathrm{mb}}\left({ }^{12} \mathrm{CO}, J=1-0\right)=$ $23 \mathrm{~K}, T_{\mathrm{mb}}\left({ }^{12} \mathrm{CO}, J=2-1\right)=63 \mathrm{~K}, T_{\mathrm{mb}}\left({ }^{13} \mathrm{CO}, J=1-0\right)=$ $1 \mathrm{~K}, T_{\mathrm{mb}}\left({ }^{13} \mathrm{CO}, J=2-1\right)=4.3 \mathrm{~K}$. We note that due to recent improvements in the beam shape, the main-beam temperatures of $1 \mathrm{~mm}$ lines appear now somewhat higher than in older observations (by factors 1.3-1.5).

The observed spectra are shown in Figs. 1 to 16. As baseline, only straight lines were subtracted.

Usually, PPNe are angularly compact objects, compared with the resolution of the telescope, and observations of a single point are enough to measure the total emission. However, the angular extent of some of the observed objects is known to be comparable to the $12-13^{\prime \prime}$ beam of the $30 \mathrm{~m}$ telescope at $230 \mathrm{GHz}$. We have performed small maps of a few objects but, due to their small size, the information on the nebula shape is poor and the main result from our maps is simply its image extent at half maximum; see Sect. 5 .

\section{Calculation of physical parameters from the CO data}

\subsection{CO emissivity and determination of the emitting mass and its momentum and energy}

We will calculate the mass emitting in a spectral range within a $\mathrm{CO}$ rotational line from the discussion on the $\mathrm{CO}$ excitation and emissivity by Bujarrabal et al. (1997) and

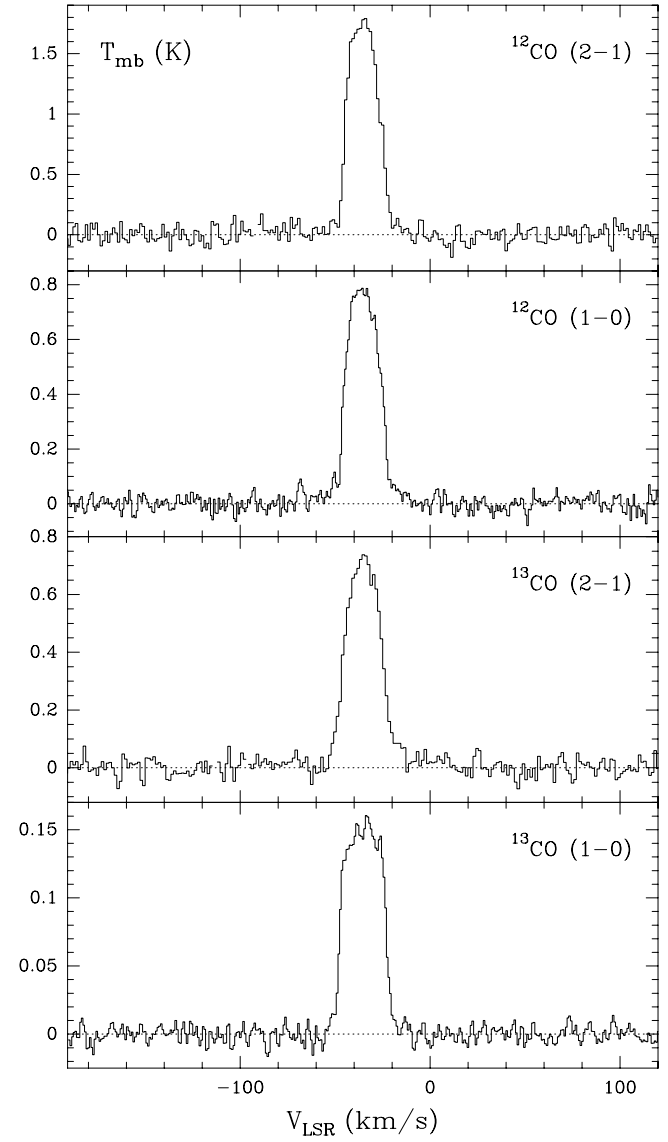

Fig. 4. CO observations of IRAS $17436+5003$.

the properties of the CO emission from well studied nebulae (Sect. 1). The possible associated errors are discussed in that paper and Sect. 4.2. Note that, when the source is significantly extended compared with the telescope resolution, a correction to the central brightness temperature proportional to the area of the beam-convolved image is necessary.

The emissivity is parametrized by the value of a (constant) rotational temperature, $T_{\text {rot }}$. In previous papers, see Bujarrabal et al. $(1997,1998 a)$ and Sánchez Contreras et al. (1997), we have shown that the characteristic rotational temperature of the $\mathrm{CO}$ emission in the best studied nebulae is low, between 10 and $30 \mathrm{~K}$, and remains significantly constant across most of the source. In those objects in which good ${ }^{12} \mathrm{CO}$ and ${ }^{13} \mathrm{CO}$ data exist, we will estimate the values of this parameter from the observed $J=2-1 / J=1-0$ line ratio; this is equivalent to take the value of $T_{\text {rot }}$ that leads to the same value of the total mass from the data of both lines (of both ${ }^{12} \mathrm{CO}$ and ${ }^{13} \mathrm{CO}$ ), see Sect. 5.1. As we will see, we systematically obtain values of $T_{\text {rot }}$ compatible with the above range. For objects in which such an estimate is not possible, we will assume $T_{\text {rot }} \sim 15 \mathrm{~K}$. The emissivities for a given $T_{\text {rot }}$ and so the conversion from $T_{\mathrm{mb}}$ to emitting mass are calculated assuming optically thin emission. At least for ${ }^{13} \mathrm{CO} J=1-0$, the optical depth is found to be lower than one in the well 


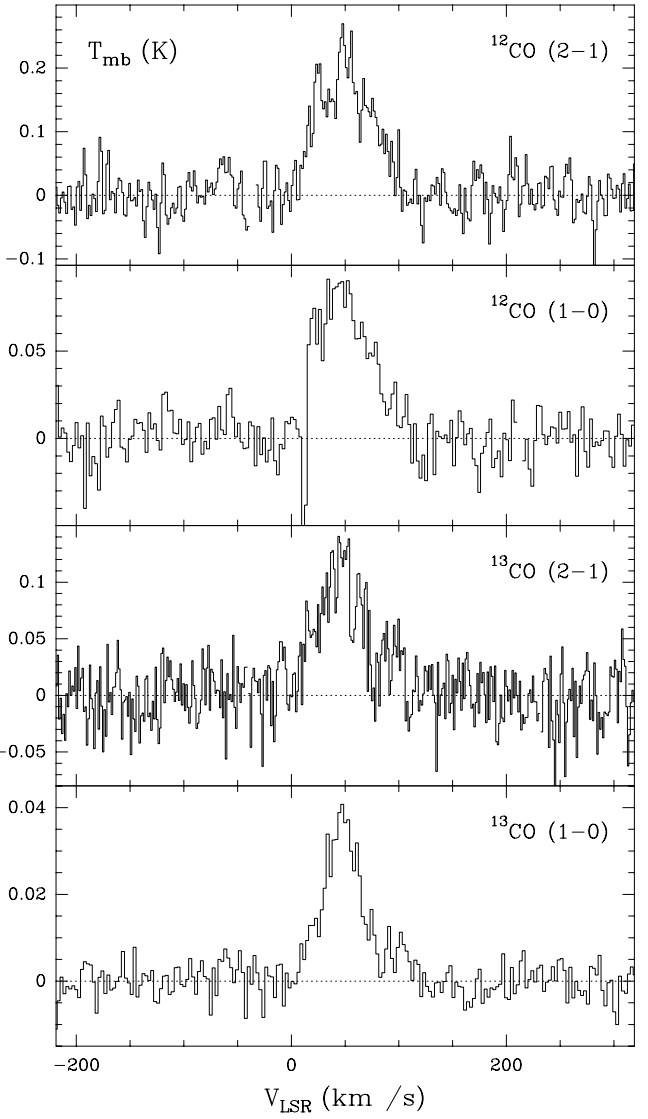

Fig. 5. CO observations of He 3-1475.

studied objects (see also 4.2); for that reason (and the larger telescope beam), this line will be preferably used.

We will adopt the relative $\mathrm{CO}$ abundances $X\left({ }^{12} \mathrm{CO}\right)=$ $2 \times 10^{-4}$ and $X\left({ }^{13} \mathrm{CO}\right)=2 \times 10^{-5}$. These relatively high values are characteristic of AGB envelopes and PPNe with strong CO emission (Bujarrabal et al. 1997, 1998a, Alcolea et al. 2001, etc.), and therefore can be safely applied to not very evolved PPNe, in which photodissociation by the stellar UV is not important yet.

The spectral sampling used in our calculations are defined in terms of LSR velocity, $V_{\mathrm{LSR}}$. We will assume that the $\mathrm{CO}$ emission in the line wings comes from more or less elongated structures in which the velocity is mainly axial, as found for M1-92, OH231.8+4.2, etc. Then, $V_{\mathrm{LSR}}$ can be converted into actual flow velocity of the emitting gas, $V$; provided that we know the inclination of the axis with respect to the plane of the sky, $i$, and that we can deduce a systemic LSR velocity, $V_{\text {sys }}$, from the CO profiles. Note that $V_{\text {sys }}$ is expected to give the movement of the center of gravity, defining the rest frame for which those parameters are calculated. Then,

$V=\left(V_{\mathrm{LSR}}-V_{\mathrm{sys}}\right) / \sin (i)$.

Once we know the mass emitting in a spectral range, $m$, and the expansion velocity corresponding to it, $V$, we can calculate the total mass, linear "momentum", $P$, and kinetic energy, $E$, by summing over the whole spectra the

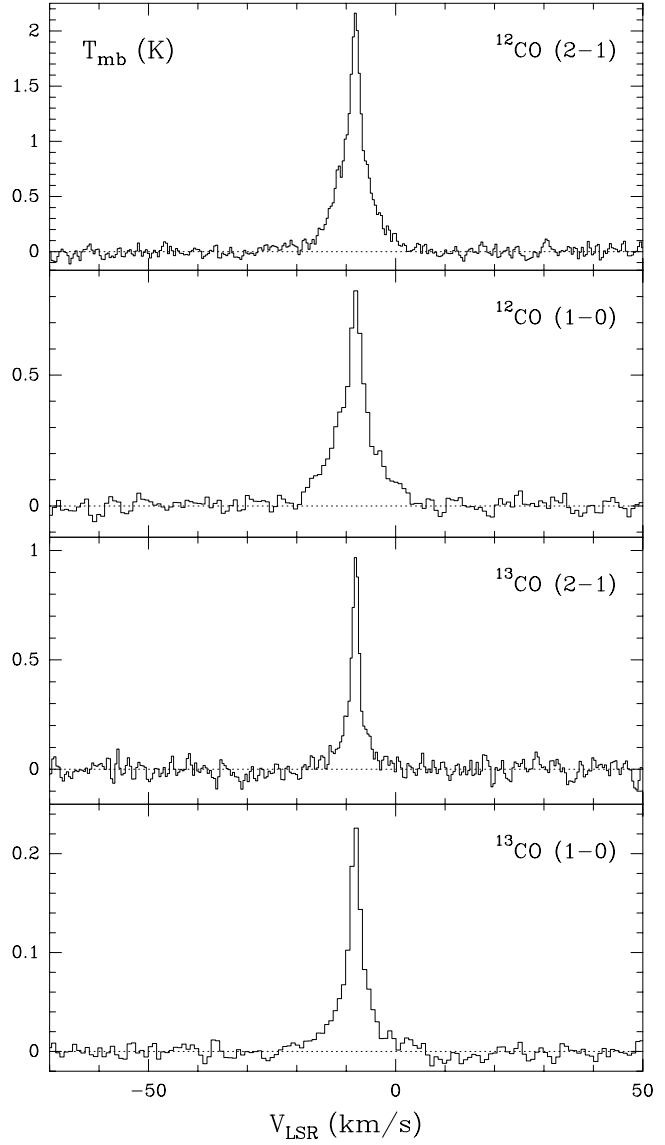

Fig. 6. CO observations of 89 Her.

values of $m$ and of the products $m|V|$ and $\frac{1}{2} m V^{2}$. So, we calculate

$P=\sum m\left(V_{\mathrm{LSR}}\right) \times\left|V_{\mathrm{LSR}}-V_{\mathrm{sys}}\right| / \sin (i)$,

and

$E=\frac{1}{2} \sum m\left(V_{\mathrm{LSR}}\right) \times\left[\left(V_{\mathrm{LSR}}-V_{\mathrm{sys}}\right) / \sin (i)\right]^{2}$.

Note that we are not exactly calculating a linear momentum, since the sum is not performed vectorially. When the flow is exactly bipolar, our method gives the sum of the moduli of the momenta in the two opposite directions. In general, we calculate sums of momentum moduli for different directions. The calculated parameter (the total "scalar momentum") is however relevant for our purposes, since it can be compared with the "momentum" carried by radiation pressure per unit time, calculated as $L / c$, which has the same meaning.

When the inclination of the nebula axis is not known, we will use $i=30^{\circ}$, as we have mentioned. This assumption is intermediate between the extreme cases, perpendicular or parallel to the plane of the sky. Note, moreover, that if we calculate the sum of the momentum moduli from observations of a spherically symmetric envelope, with constant radial velocity, we find exactly the same formula as for a bipolar flow with $i=30^{\circ}$, i.e.:

$P_{\text {isot }}=2 \sum m\left(V_{\mathrm{LSR}}\right) \times\left|V_{\mathrm{LSR}}-V_{\mathrm{sys}}\right|=P_{i=30}$. 


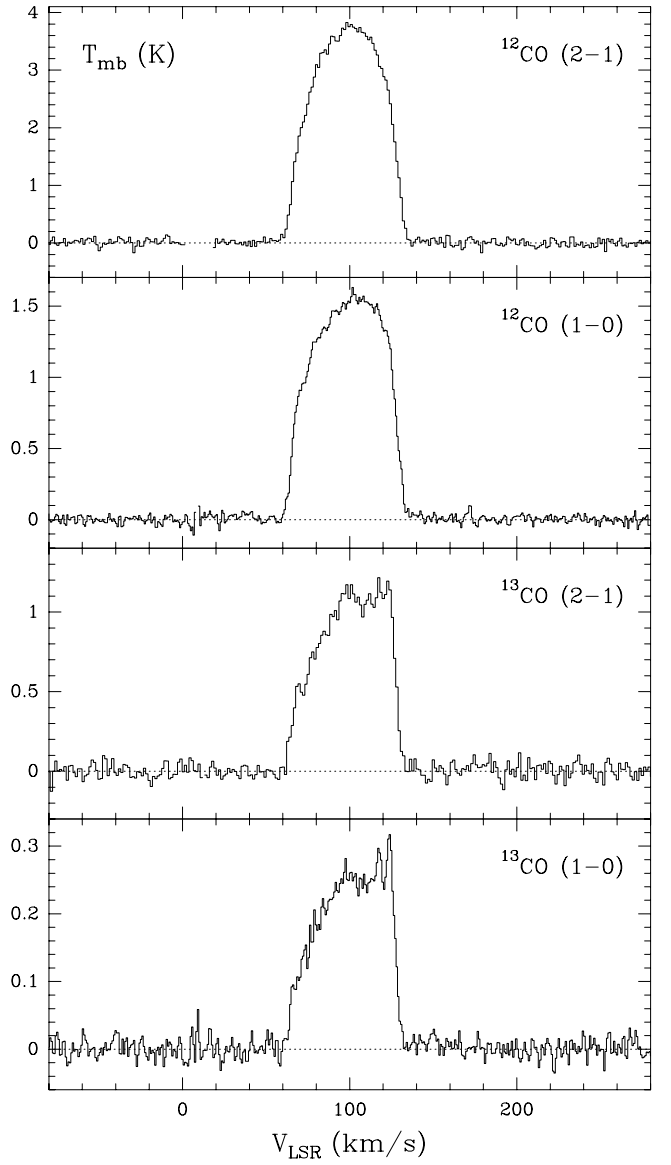

Fig. 7. CO observations of AFGL 2343.

In this isotropical case,

$E_{\text {isot }}=3 \frac{1}{2} \sum m\left(V_{\mathrm{LSR}}\right) \times\left(V_{\mathrm{LSR}}-V_{\mathrm{sys}}\right)^{2}=\frac{3}{4} E_{i=30}$.

Note that the total mass calculation only depends on the velocity integrated intensity, it is therefore independent of the assumed source geometry.

For all possible density and velocity distributions (keeping the value of $V_{\text {sys }}$ as that of the reference frame), there is a lower limit for the momentum and energy: those calculated assuming bipolar ejections with $i=90^{\circ}$, i.e. in the line of sight:

$$
\begin{aligned}
& P_{\min }=\sum m\left(V_{\mathrm{LSR}}\right) \times\left|V_{\mathrm{LSR}}-V_{\mathrm{sys}}\right|=\frac{1}{2} P_{i=30}, \\
& E_{\mathrm{min}}=\frac{1}{2} \sum m\left(V_{\mathrm{LSR}}\right) \times\left(V_{\mathrm{LSR}}-V_{\mathrm{sys}}\right)^{2}=\frac{1}{4} E_{i=30} .
\end{aligned}
$$

The minimum momentum is just one half of that given by our previous isotropic and $i=30^{\circ}$ approximations.

There are other possible models for the mass and velocity distributions, but in general all them give similar results for the momentum and energy to those presented here. We can see more detailed source descriptions and the derived results in e.g. Bujarrabal et al. (1998a) and Alcolea et al. (2001), based on their high-quality CO maps. We also mention the disk model used in Sahai et al. (2000) for the PPN Frosty Leo. In this model the gas flows in a

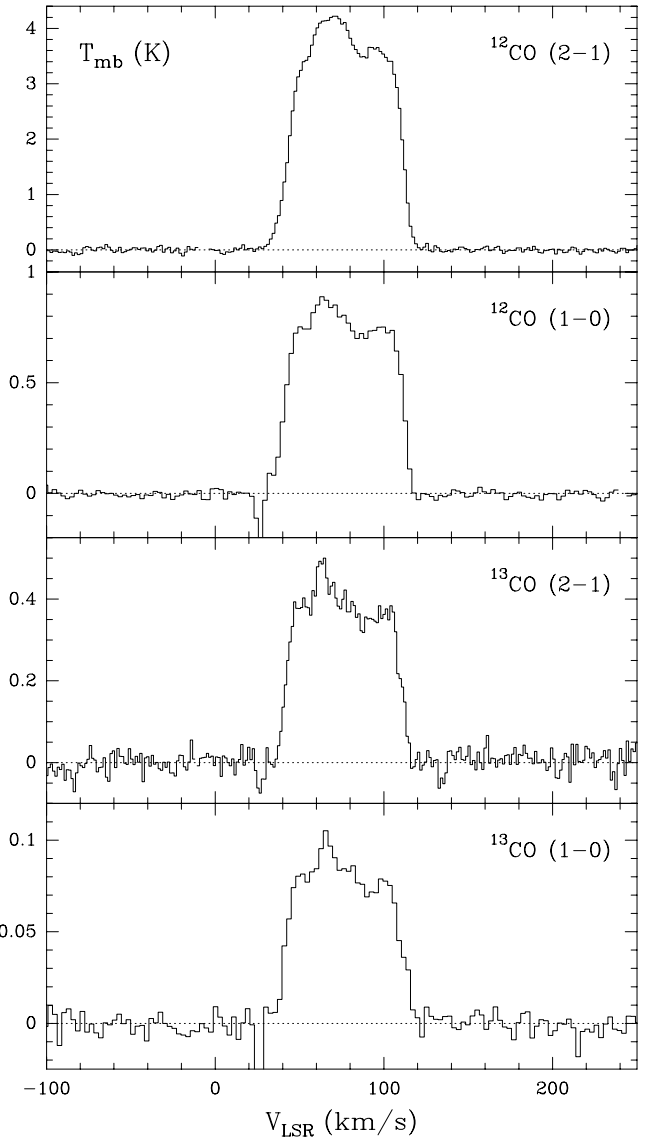

Fig. 8. CO observations of IRC +10420 .

plane perpendicular to the nebula symmetry axis at constant velocity; the axis inclination is again the basic geometric parameter. The expansion velocity is given in this model by the maximum of the relative observed velocity, $V_{\mathrm{LSR}}-V_{\text {sys }}$, divided by $\cos (i)$. The resulting momentum and energy expression are slightly more complex than in the axial-flow case, but the results do not differ from those of the other simple models (see Sect. 4.2 and Sahai et al.).

Only when the gas velocity is assumed to be closely parallel to the plane of the sky (for instance in a bipolar flow with $i \sim 0^{\circ}$ or in a disk with the axis close to the line of sight), the corrections needed to convert the observational parameters to momentum and energy are very high. In the objects treated here we never assumed values of $i$ smaller than $15^{\circ}$ (Tables 1,2).

As we have mentioned, PPNe often show different kinematical components. In several sources, there is clearly a central low-velocity feature in the CO profiles. In the well studied cases (see Sect. 8.1), this central spectral component is known to come from a spherical slowly expanding envelope or from a disk/torus perpendicular to the nebula axis. In these sources, the line core is thought to represent the part of the AGB envelope that has not been affected by the wind interaction characteristic of the protoplanetary evolution. Therefore we must treat separately the line wings and the line core. For the line wings, the most interesting spectral feature for us, we will in general assume the 


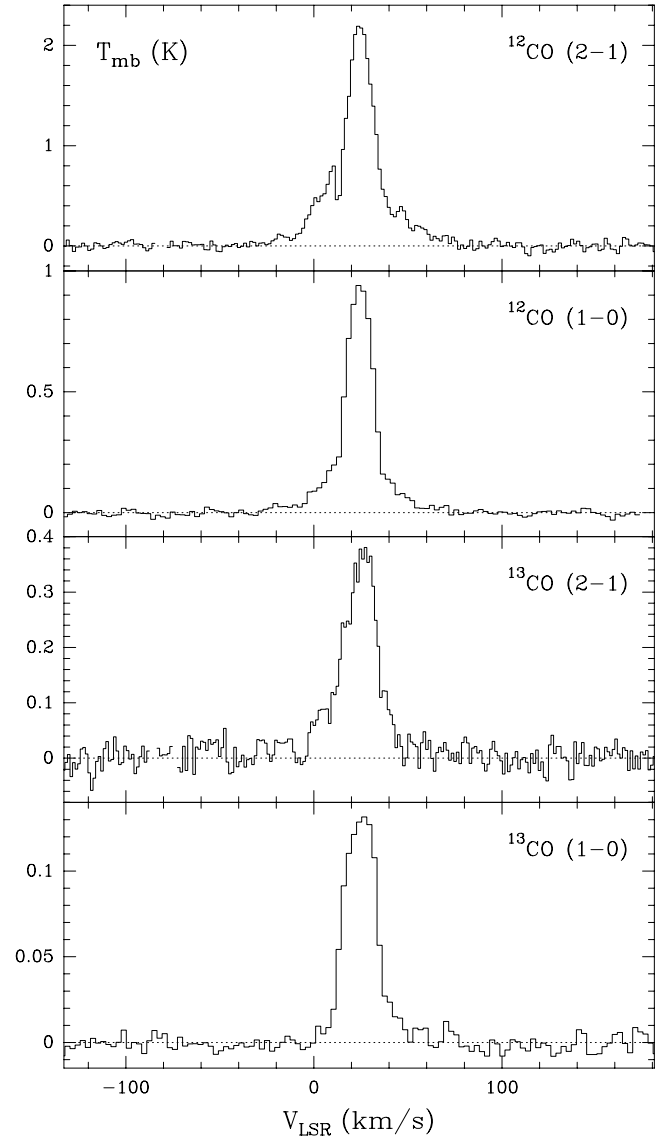

Fig. 9. CO observations of IRAS 19500-1709.

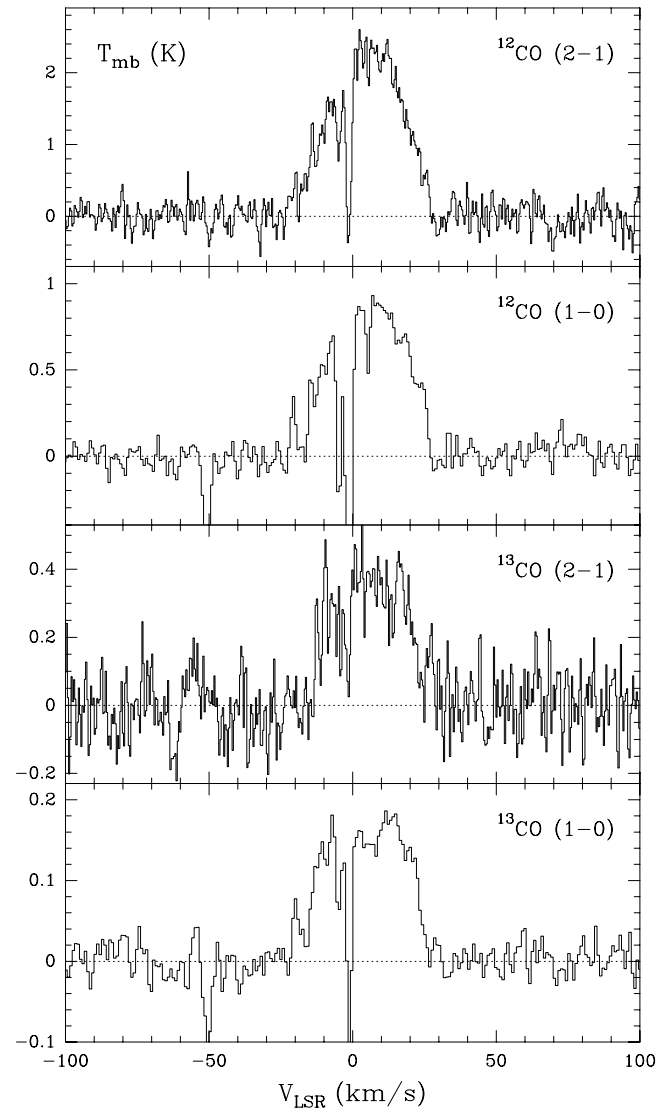

Fig. 10. CO observations of CRL 2477.

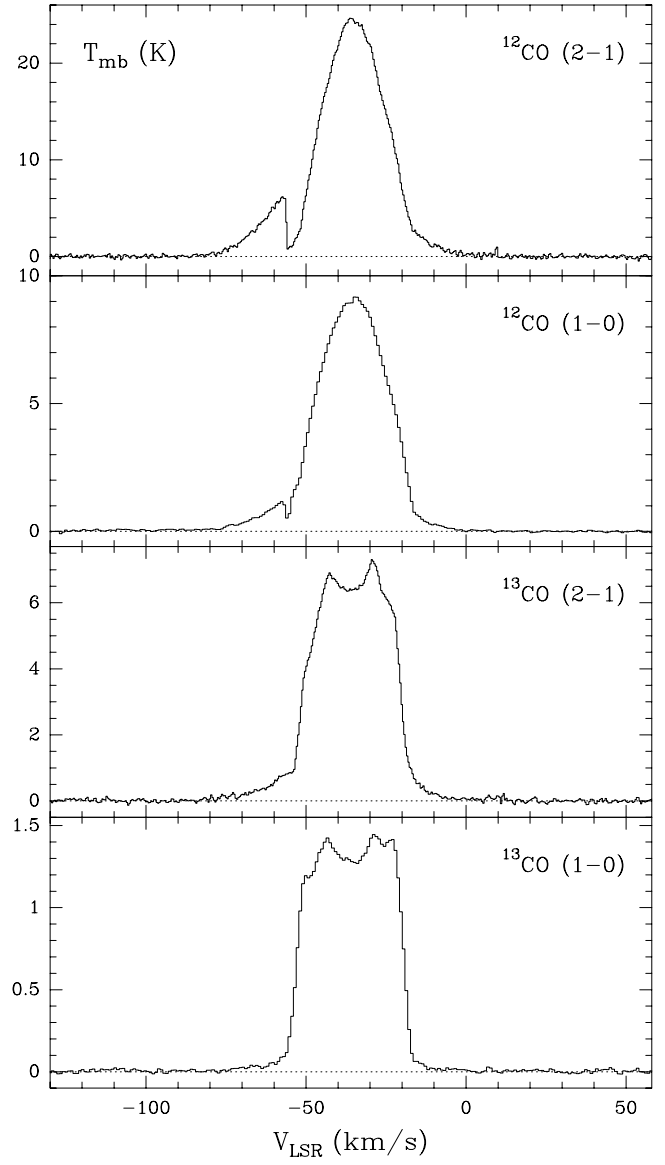

Fig. 11. CO observations of CRL 2688.

bipolar flow model, as discussed above. Except for some well studied cases mentioned in Sect. 5, for the line core we will assume isotropic expansion and spherical symmetry, since these values will be in that way more directly comparable to those usually found for AGB envelopes.

\subsection{Uncertainties in the calculation}

The assumptions listed in Sect. 4.1 could lead, if they are not satisfied, to errors. In a previous work (Bujarrabal et al. 1997), we have already discussed these error sources. In this paper we will adapt this discussion, without repeating all details, to the models used here.

1. We are assuming optically thin emission, at least in the ${ }^{13} \mathrm{CO} J=1-0$ transition. The main reason to believe that this hypothesis is reasonable is that the ${ }^{13} \mathrm{CO}$ lines are in all cases significantly weaker than the ${ }^{12} \mathrm{CO}$ lines, in spite of the similar excitation properties of both molecules and the similar extents found from both theoretical reasoning (i.e. from photodissociation theory, Mamon et al. 1988) and observational results (see maps by Bujarrabal et al. 1998a; Sánchez Contreras et al. 1997). Under these conditions, ${ }^{13} \mathrm{CO} /{ }^{12} \mathrm{CO}$ line ratios much lower than 1 can only be explained if at least the ${ }^{13} \mathrm{CO}$ lines are optically thin. Nevertheless, if these lines were opaque, our calculations of mass, momentum and energy would lead to underestimates of the true values (but never overestimates); 


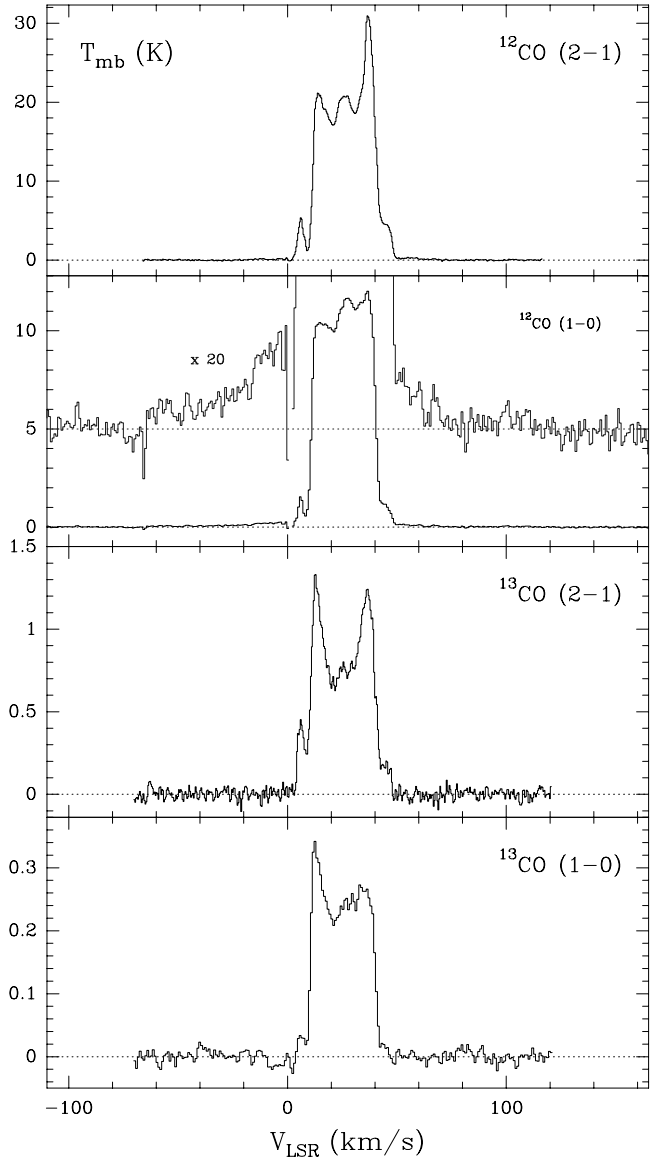

Fig. 12. CO observations of NGC 7027 .

in any case the above discussion strongly suggests that the possible underestimate is moderate, mainly in the relatively weak line wings and well studied objects.

2. We also assume a relatively low rotational temperature $(\sim 15 \mathrm{~K})$, that is used to parametrize the CO line excitation. As we have discussed in Sect. 4.1, such low values are found in the PPNe in which the CO line emission has been well observed. Note that rotational temperatures larger than $30 \mathrm{~K}$ (if the emission is not resolved by the telescope and optically thin) would lead to values of the brightness temperature ratio ${ }^{13} \mathrm{CO} J=2-1 / J=1-0 \gtrsim$ 12 , which is certainly not observed. As we have discussed in Bujarrabal et al. (1997), the emissivity in the observed lines for this low $T_{\text {rot }}$ is close to the absolute maximum. Only overestimates by less than a factor 2 in the mass, momentum and energy are then expected due to errors in the excitation treatment. Of course, significant underestimates are possible if $T_{\text {rot }}$ is in fact very large; the underestimate for $T_{\text {rot }}=40 \mathrm{~K}$ is by about a factor 3 . We have mentioned that the mass of the hot gas is small (Sect. 1); if a massive warm componet exist, we would again underestimate the total mass and momentum.

Bujarrabal et al. (1997) calculated the effects of departures from thermalization in the CO level population. For the high number densities calculated in well observed nebulae, $\gtrsim 10^{4} \mathrm{~cm}^{-3}$ (Bujarrabal et al. 1998a; Alcolea et al. 2001), the relevant lines are almost thermalized and only

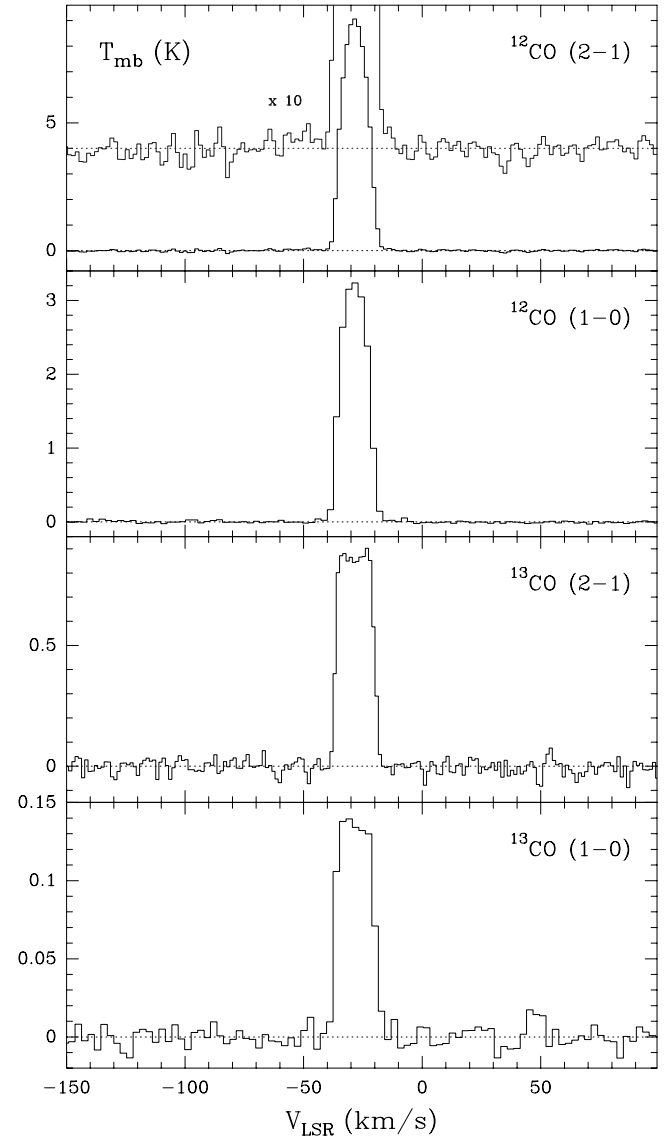

Fig. 13. CO observations of IRAS $22272+5435$.

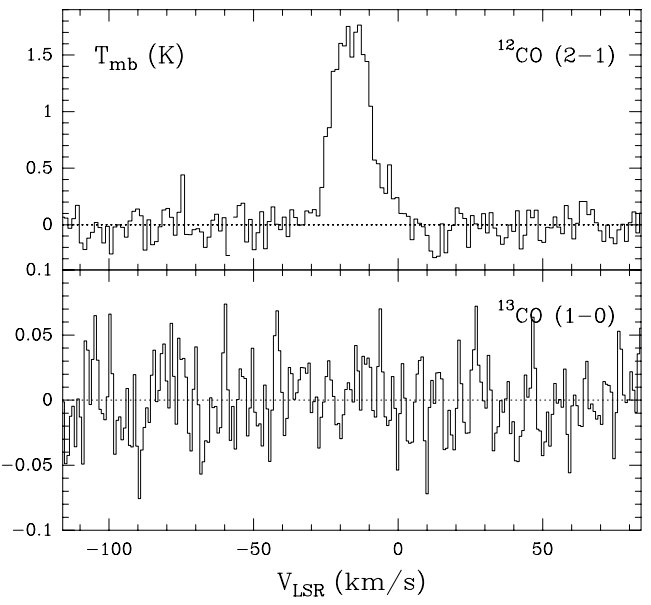

Fig. 14. CO observations of IRAS $23304+6147$.

small corrections to the emissivity are expected. In any case, even for extreme departures the possible overestimates would not exceed a factor 1.5 .

The case of the PPN IRAS 22272+5435 illustrates the moderate uncertainties that one can expect in our calculations due to the excitation assumptions. In Sect. 5.1.12 and Table 3, we present results of the molecular mass of this source, for which some indication exists that the observed lines come from an envelope similar to that of an AGB star. We calculate the molecular mass and the dynamical parameters assuming three models for the $\mathrm{CO}$ 


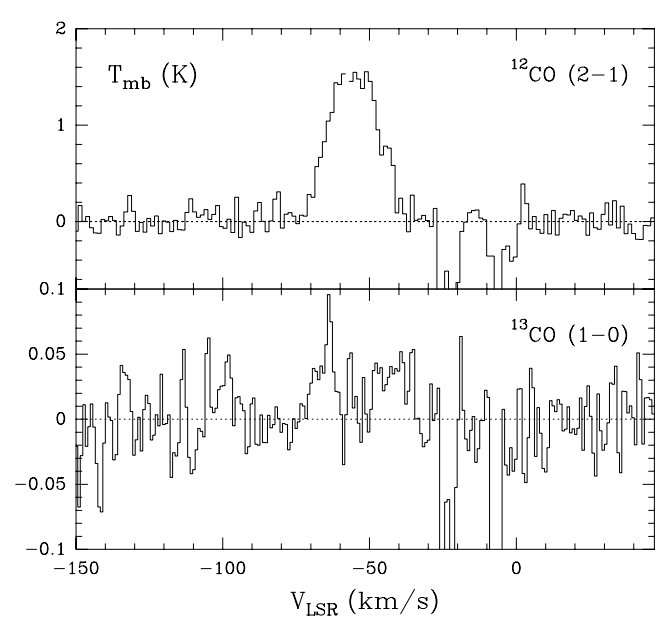

Fig. 15. CO observations of IRAS $23321+6545$.

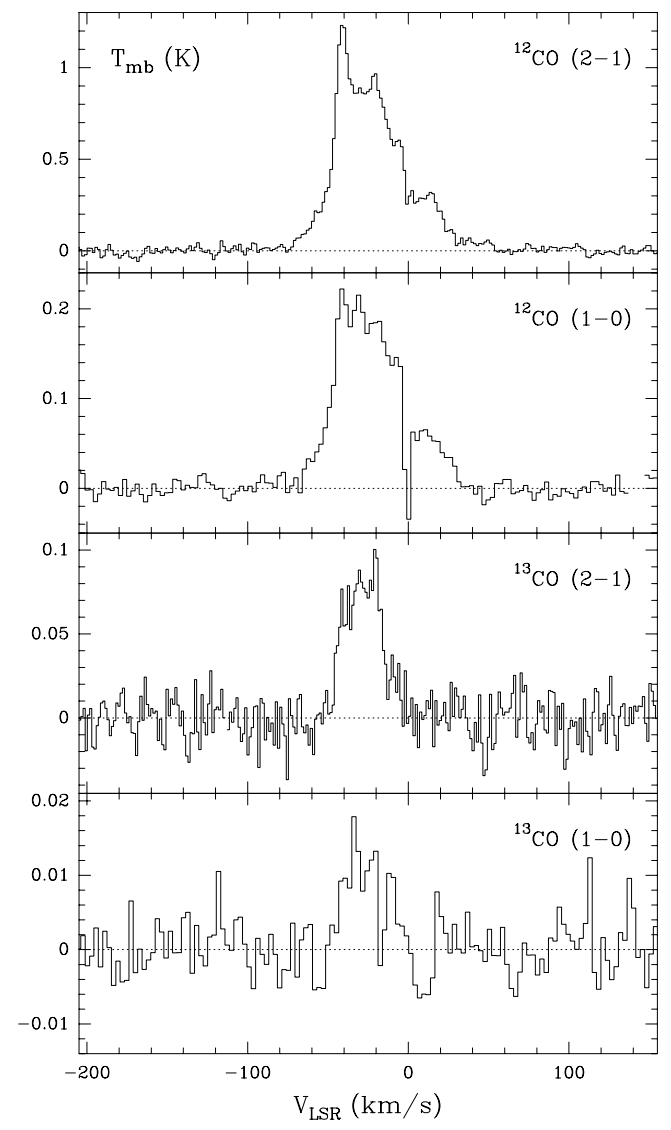

Fig. 16. CO observations of M 2-56.

excitation: our standard model for spherical-isotropic expansion (with $T_{\text {rot }}=15 \mathrm{~K}$ ), our model for sphericalisotropic expansion and assuming $T_{\text {rot }}=25 \mathrm{~K}$ (a value that, as we will see, may be present in some PPNe), and assuming that the lines come from an envelope similar in density, abundance and temperature distribution to those of AGB stars. As we see in Table 3, the results are comparable for the different models. (It is well known that $\mathrm{CO}$ data analysis provides robust ways to determine the mass in the CO-rich envelopes, due to the low dependence of the results on the excitation conditions and general assumptions, see e.g. Bujarrabal 1999.)

3. We are taking a relatively high value of the ${ }^{13} \mathrm{CO}$ relative abundance, $X\left({ }^{13} \mathrm{CO}\right)=2 \times 10^{-5}$, as usually found in molecule-rich PPNe and AGB envelopes (Kahane et al. 1992; Bujarrabal et al. 1994). Such a value of $X\left({ }^{13} \mathrm{CO}\right)$ almost corresponds to the maximum number of ${ }^{13} \mathrm{CO}$ molecules that can be formed for the expected ${ }^{13} \mathrm{C}$ abundance. As discussed by Bujarrabal et al. (1997), some PPNe are known to show relatively low molecular abundances, probably due to photodissociation by the UV photons emitted by the (already) warm central star. Therefore, again we can hardly expect strongly overestimated values of the derived parameters from the possible errors in the assumed $X\left({ }^{13} \mathrm{CO}\right)$ value. However, if in some source the molecular photodissociation is very efficient we would severely underestimate the mass, as well as the other dynamical parameters. It is difficult to limit such an effect. We hope that the fact that our sample mostly includes intense sources in molecular emission implies that their molecular component includes most of the nebular mass; this is known to be case in well studied nebulae like CRL 2688, CRL 618, M 1-92, OH 231.8+4.2, etc. In any case, we must keep in mind that the parameters derived here always refer to the (cool) molecular component of PPNe.

4. We have also mentioned in Sect. 4.1 that the uncertainty in the nebula structure and kinematics affects the determination of the momentum and energy (not of the total mass). When we use a bipolar jet model with inclination $i$ (values listed in Tables 1,2), the maximum possible overestimate of the momentum due to errors in the inclinations is by a factor $1 / \sin (i)$. For the discussed sources, for which we always take $i \geq 15^{\circ}$, this factor does not exceed 3.9. The energy measurement is more uncertain, it can be overestimated by a factor $1 / \sin ^{2}(i)$. When the model source is spherical with isotropic flow, the maximum overestimate of $P$ is by a factor 2 (3 for the energy). For most sources, the inclination of the axis is relatively well known, so significantly smaller errors are expected. For the others we adopt $i=30^{\circ}$, the maximum overestimate of $P$ is then by a factor 2 . On the other hand, if the true inclination of the mass flow with respect to the plane of the sky is close to $0^{\circ}$, we may strongly underestimate $P$ and $E$. The probability that $i$ is smaller than $15^{\circ}$, assuming a random distribution of the nebula axes, is 0.26 . Therefore, for $\sim 75 \%$ of the sources with uncertain inclination, the underestimate of the momentum is smaller than a factor $\sim 2$. It is possible that in some object the momentum underestimate is significantly stronger.

Note that this discussion holds almost exactly if the velocity/mass distributions are more complex than assumed.

In Sect. 4.1 we discuss calculations for one object, Frosty Leo, assuming very different geometries, namely: our standard bipolar flow, a spherical-isotropic expansion, and a disk-like distribution (see results in Table 3). We discuss the case of this source because its well known image in the optical could suggest very different configurations 
for the CO cloud, see 5.1.3. As we see, the differences between the results for such different geometries are small, provided that the inclination of the flow with respect to the plane of the sky is not very small.

As we have mentioned, the distance to some sources is quite uncertain. But we note that the parameters derived from the molecular observations (mass, linear momentum and kinetic energy of the flows) depend on $D^{2}$, in the same way as the stellar total luminosity and the momentum carried by it. Therefore, our main result, the comparison between the gas outflow momentum (energy) and the momentum (energy) carried by the stellar radiation, is not directly affected by the errors in the distance.

\section{Mass, momentum and energy carried by PPN outflows}

\subsection{Sources observed by us in CO emission}

The mass, linear momentum and kinetic energy of the different nebular components calculated in the objects observed by us, following the methods described in Sect. 4, are summarized in Table 3. In this section, we discuss the parameters used for each nebula. The adopted values for the axis inclination $i$ and the distance $D$ are listed in Table 1 (Sect. 2). We will also discuss (mainly for the first sources) the errors and limits due to the CO data uncertainties, as well as to the excitation and source models. In Sect. 5.2 we discuss the calculations for sources observed in CO by other authors; the difference of the treatment is due to the different quality of the data, the results, as we will see, are very similar for both groups.

\subsubsection{IRAS $04296+3429$}

Our spectra (Fig. 1, Sect. 3) show a central component and weak wings, that are not seen in the ${ }^{13} \mathrm{CO} J=1-0$ line. We deduced from inspection of the ${ }^{12} \mathrm{CO} J=2-1$ line that the slow component is characterized by a systemic velocity $V_{\text {sys }}=-65 \mathrm{~km} \mathrm{~s}^{-1}(\mathrm{LSR})$ and a velocity range equal to $-65 \pm 11 \mathrm{~km} \mathrm{~s}^{-1}$. The very compact HST image $\left(2-3^{\prime \prime}\right.$ wide, Sahai et al. 2000) suggests that the nebula is much smaller than the resolution of our telescope, as recently confirmed by unpublished CO maps of this source. The CO line intensity ratios are compatible with the range of low rotational temperatures assumed in our standard model $(10-20 \mathrm{~K})$. We will accordingly apply our standard procedure to the calculation of the mass, momentum and kinetic energy in this source, using the ${ }^{12} \mathrm{CO} J=1-0$ line and a bipolar outflow model (with $i=25^{\circ}$ ) for the weak line wings, and using the ${ }^{13} \mathrm{CO} J=1-0$ line and a spherical model for the line core. We have checked that, at least for the line core, both ${ }^{12} \mathrm{CO}$ and ${ }^{13} \mathrm{CO} J=1-0$ lines would produce very similar results, which is a consistency proof of the assumed excitation, abundances and low opacities. The results obtained for the fast component are in any case uncertain, due to its weak emission. The distance to this source, see Table 1 , is poorly known, but this should not affect our main results (Sect. 4.2).

The results of the calculations with this model are given in Table 3 . Since the fast outflow is assumed to have $i=25^{\circ}$, the lower limit to its momentum (for an unrealistic flow in the direction of the line of sight, see Sect. 4.2) would be 2.4 times smaller than the value in the table. Calculations with a spherical-isotropic model for the fast gas would yield a momentum about 1.2 times smaller that the adopted result.

\subsubsection{CRL 618}

As we see in Fig. 2, the CO lines of CRL 618 show a clearly composite structure, with a central strong core and wide wings. From existent mapping observations of the central component (e.g. Bujarrabal et al. 1988), it is known that this spectral feature comes from a quasispherical structure extended about 15 arcsec. The wide line wings, on the other hand, are known to come from a bipolar inner flow (Neri et al. 1992) extended less than $5^{\prime \prime}$. Therefore, the spatial resolution of our CO $J=1-$ 0 single-dish observations, $\sim 22^{\prime \prime}$, is wide enough to collect all its flux. We deduced from our spectra a systemic velocity $V_{\text {sys }}=-21 \mathrm{~km} \mathrm{~s}^{-1}$ and an expansion velocity for the central component equal to $17.5 \mathrm{~km} \mathrm{~s}^{-1}$. In this case it is therefore fully justified to use a spherical model for the line core and a bipolar fast flow (with $i=45^{\circ}$ ) for the line wings (Sect. 4). The wings are very weak in the ${ }^{13} \mathrm{CO}$ lines; therefore, we calculated the parameters for them from the ${ }^{12} \mathrm{CO}$ lines (mainly $J=1-0$ ). For a spherical-isotropic model, the momentum calculated for the fast outflow would be 1.4 times larger than the value for our standard bipolar model; the minimum momentum (if the material flows in the direction of the line of sight) would be 1.4 times smaller.

Some authors (see Bujarrabal et al. 1988 and references therein) have proposed somewhat larger rotational temperatures in this source than those found in other PPNe. For the line core, the rotational temperature derived from the ${ }^{13} \mathrm{CO} J=2-1 / J=1-0$ brightness ratio is $\sim 25 \mathrm{~K}$, the correction factor with respect to our standard estimate (for $T_{\text {rot }}=15 \mathrm{~K}$ ) is only $\sim 1.4$. The $\left({ }^{12} \mathrm{CO}\right)$ line wings suggest a low excitation temperature and the correction factor is still smaller. Therefore, we will only apply the above correction to the central spectral component.

\subsubsection{Frosty Leo}

The CO spectra of Frosty Leo show a central intense feature that will be taken as the slow component, with $V_{\text {sys }}=$ $-11 \mathrm{~km} \mathrm{~s}^{-1}$ and an expansion velocity of $10 \mathrm{~km} \mathrm{~s}^{-1}$. The line wings in this source are very conspicuous at all wavelengths, but being much weaker in ${ }^{13} \mathrm{CO}$, which suggests optically thin emission at least in the ${ }^{13} \mathrm{CO}$ lines. We performed small maps that show that the source is not resolved in $\mathrm{CO}$ emission, with an extent $\lesssim 5^{\prime \prime}$. 
Table 3. Calculations of the mass, momentum and kinetic energy for the sources observed in CO by us.

\begin{tabular}{|c|c|c|c|c|c|}
\hline source & $\begin{array}{c}\text { mass } \\
M\left(M_{\odot}\right)\end{array}$ & $\begin{array}{l}\text { momentum } \\
P\left(\mathrm{~g} \mathrm{~cm} \mathrm{~s}^{-1}\right)\end{array}$ & $\begin{array}{l}\text { kinetic energy } \\
E(\mathrm{erg})\end{array}$ & $\begin{array}{l}\frac{P}{L / c} \\
(\mathrm{yr}) \\
\end{array}$ & comments \\
\hline IRAS $04296+3429$ & $\begin{array}{ll}0 \\
12\end{array}$ & $25 \times 10^{38}$ & $13 \times 10^{44}$ & $9 \times 10^{3}$ & $L / c=2.8 \times 10^{34} \mathrm{~g} \mathrm{~cm} \mathrm{~s}^{-1} \mathrm{yr}^{-1}$ \\
\hline $\begin{array}{l}\text { Siow component } \\
\text { fast outflow }\end{array}$ & $3.7 \times 10^{-3} ?$ & $\begin{array}{l}2.5 \times 10 \\
3.3 \times 10^{37} ?\end{array}$ & $\begin{array}{l}1.3 \times 10 \\
7.7 \times 10^{43} ?\end{array}$ & $1.2 \times 10^{3} ?$ & from ${ }^{12} \mathrm{CO} J=1-0$, uncertain analysis \\
\hline CRL 618 & & & & & $L / c=1.2 \times 10^{35} \mathrm{~g} \mathrm{~cm} \mathrm{~s}^{-1} \mathrm{yr}^{-1}$ \\
\hline slow component & 0.65 & $2.1 \times 10^{39}$ & $1.8 \times 10^{45}$ & $1.8 \times 10^{4}$ & assuming $T_{\text {rot }}=25 \mathrm{~K}$ \\
\hline fast outflow & 0.045 & $8.4 \times 10^{38}$ & $5.2 \times 10^{45}$ & $7 \times 10^{3}$ & from ${ }^{12} \mathrm{CO} J=1-0$ \\
\hline Frosty Leo & & & & & $L / c=1.1 \times 10^{34} \mathrm{~g} \mathrm{~cm} \mathrm{~s}^{-1} \mathrm{yr}^{-1}$ \\
\hline slow component & 0.36 & $8.0 \times 10^{38}$ & $4.5 \times 10^{44}$ & $7 \times 10^{4}$ & \\
\hline fast outflow & 0.56 & $9.0 \times 10^{39}$ & $4.0 \times 10^{46}$ & $8 \times 10^{5}$ & bipolar model \\
\hline fast outflow & 0.60 & $4.6 \times 10^{39}$ & $8.1 \times 10^{45}$ & $4.2 \times 10^{5}$ & spherical-isotropic model \\
\hline fast outflow & 0.56 & $6.5 \times 10^{39}$ & $1.1 \times 10^{46}$ & $6 \times 10^{5}$ & disk, constant radial velocity \\
\hline IRAS $17436+5003$ & & & & & $L / c=2.4 \times 10^{35} \mathrm{~g} \mathrm{~cm} \mathrm{~s}^{-1} \mathrm{yr}^{-1}$ \\
\hline slow component & 0.57 & $1.2 \times 10^{39}$ & $6.2 \times 10^{44}$ & $5 \times 10^{3}$ & \\
\hline fast outflow & 0.11 & $6.1 \times 10^{38}$ & $8.6 \times 10^{44}$ & $2.5 \times 10^{3}$ & weak wings \\
\hline Нe $3-1475$ & & & & & $L / c=3.6 \times 10^{34} \mathrm{~g} \mathrm{~cm} \mathrm{~s}^{-1} \mathrm{yr}^{-1}$ \\
\hline slow component & 0.16 & $2.5 \times 10^{38}$ & $1.1 \times 10^{44}$ & $7 \times 10^{3}$ & \\
\hline fast outflow & 0.47 & $1.8 \times 10^{39}$ & $3.1 \times 10^{45}$ & $5 \times 10^{4}$ & \\
\hline 89 Her & & & & & $L / c=1.3 \times 10^{34} \mathrm{~g} \mathrm{~cm} \mathrm{~s}^{-1} \mathrm{yr}^{-1}$ \\
\hline slow component & $3.3 \times 10^{-3}$ & $2.2 \times 10^{36}$ & $3.8 \times 10^{41}$ & $1.7 \times 10^{2}$ & \\
\hline fast outflow & $1.0 \times 10^{-3}$ & $2.9 \times 10^{36}$ & $1.7 \times 10^{42}$ & $2.2 \times 10^{2}$ & \\
\hline AFGL 2343 & & & & & $L / c=2.3 \times 10^{36} \mathrm{~g} \mathrm{~cm} \mathrm{~s}^{-1} \mathrm{yr}^{-1}$ \\
\hline unique, fast component & 4.8 & $2.8 \times 10^{40}$ & $4.4 \times 10^{46}$ & $1.2 \times 10^{4}$ & spherical envelope \\
\hline $\begin{array}{l}\text { IRC }+10420 \\
\text { unique,fast component }\end{array}$ & 2.1 & $1.5 \times 10^{40}$ & $2.6 \times 10^{46}$ & $5 \times 10^{3}$ & $\begin{array}{l}L / c=2.8 \times 10^{36} \mathrm{~g} \mathrm{~cm} \mathrm{~s}^{-1} \mathrm{yr}^{-1} \\
\text { spherical envelope; extended }\end{array}$ \\
\hline IRAS 19500-1709 & & & & & $L / c=6.1 \times 10^{33} \mathrm{~g} \mathrm{~cm} \mathrm{~s}^{-1} \mathrm{yr}^{-1}$ \\
\hline slow component & 0.026 & $5.0 \times 10^{37}$ & $2.5 \times 10^{43}$ & $8 \times 10^{3}$ & \\
\hline fast outflow & $6.7 \times 10^{-3}$ & $5.3 \times 10^{37}$ & $1.4 \times 10^{44}$ & $9 \times 10^{3}$ & \\
\hline CRL 2477 & & & & & $L / c=1.6 \times 10^{34} \mathrm{~g} \mathrm{~cm} \mathrm{~s}^{-1} \mathrm{yr}^{-1}$ \\
\hline unique, fast component & 0.11 & $4.4 \times 10^{38}$ & $6.1 \times 10^{44}$ & $2.8 \times 10^{4}$ & bipolar outflow (?) \\
\hline CRL 2688 & & & & & $L / c=1.0 \times 10^{35} \mathrm{~g} \mathrm{~cm} \mathrm{~s}^{-1} \mathrm{yr}^{-1}$ \\
\hline slow component & 0.69 & $2.2 \times 10^{39}$ & $1.7 \times 10^{45}$ & $2.2 \times 10^{4}$ & \\
\hline fast outflow & 0.062 & $9.6 \times 10^{38}$ & $3.9 \times 10^{45}$ & $1.0 \times 10^{4}$ & bipolar model; $i=15^{\circ}$ \\
\hline fast outflow & 0.062 & $5.0 \times 10^{38}$ & $7.8 \times 10^{44}$ & $5 \times 10^{3}$ & spherical model \\
\hline NGC 7027 & & & & & $L / c=4.0 \times 10^{34} \mathrm{~g} \mathrm{~cm} \mathrm{~s}^{-1} \mathrm{yr}^{-1}$ \\
\hline main component & 0.60 & $1.8 \times 10^{39}$ & $1.3 \times 10^{45}$ & $4.5 \times 10^{4}$ & from ${ }^{12} \mathrm{CO} J=1-0$; extended \\
\hline main component & 0.17 & $5.2 \times 10^{38}$ & $4.0 \times 10^{44}$ & $1.3 \times 10^{4}$ & from ${ }^{13} \mathrm{CO} J=1-0$ \\
\hline very fast outflow & 0.033 & $3.7 \times 10^{38}$ & $8.5 \times 10^{44}$ & $9 \times 10^{3}$ & from ${ }^{12} \mathrm{CO} J=1-0$; spherical model \\
\hline IRAS $22272+5435$ & & & & & $L / c=3.3 \times 10^{34} \mathrm{~g} \mathrm{~cm} \mathrm{~s}^{-1} \mathrm{yr}^{-1}$ \\
\hline unique, slow component & 0.14 & $2.6 \times 10^{38}$ & $1.2 \times 10^{44}$ & $8 \times 10^{3}$ & spherical envelope; extended \\
\hline unique, slow component & 0.20 & $3.6 \times 10^{38}$ & $1.7 \times 10^{44}$ & $1.1 \times 10^{4}$ & spherical envelope; $T_{\text {rot }}=25 \mathrm{~K}$ \\
\hline unique, slow component & 0.18 & $2.9 \times 10^{38}$ & $1.2 \times 10^{44}$ & $9 \times 10^{3}$ & AGB envelope model \\
\hline no fast outflow detected & & $<6.1 \times 10^{37}$ & & $<1.8 \times 10^{3}$ & \\
\hline IRAS $23304+6147$ & & & & & $L / c=4.0 \times 10^{33} \mathrm{~g} \mathrm{~cm} \mathrm{~s}^{-1} \mathrm{yr}^{-1}$ \\
\hline slow component & $5.9 \times 10^{-3}$ & $9.5 \times 10^{36}$ & $1.3 \times 10^{42}$ & $2.4 \times 10^{3}$ & from ${ }^{12} \mathrm{CO} J=2-1$, underestimate? \\
\hline fast component & $8.0 \times 10^{-4}$ & $2.1 \times 10^{36}$ & $1.4 \times 10^{42}$ & $5.3 \times 10^{2}$ & from ${ }^{12} \mathrm{CO} J=2-1$, underestimate? \\
\hline IRAS $23321+6545$ & & & & & $L / c=2.3 \times 10^{33} \mathrm{~g} \mathrm{~cm} \mathrm{~s}^{-1} \mathrm{yr}^{-1}$ \\
\hline unique, fast component & 0.014 & $6.0 \times 10^{37}$ & $5.9 \times 10^{43}$ & $2.6 \times 10^{4}$ & bipolar (?); $i=30^{\circ}, \mathrm{D}=1 \mathrm{kpc}(?)$ \\
\hline M 2-56 & & & & & $L / c=4.0 \times 10^{34} \mathrm{~g} \mathrm{~cm} \mathrm{~s}^{-1} \mathrm{yr}^{-1}$ \\
\hline slow component & 0.046 & $1.3 \times 10^{38}$ & $9.5 \times 10^{43}$ & $3.3 \times 10^{3}$ & from ${ }^{12} \mathrm{CO} J=1-0$ \\
\hline fast component & 0.059 & $1.3 \times 10^{39}$ & $8.7 \times 10^{45}$ & $3.3 \times 10^{4}$ & from ${ }^{12} \mathrm{CO} J=1-0$ \\
\hline
\end{tabular}

The ${ }^{13} \mathrm{CO} J=2-1 / J=1-0$ line ratio indicates a rotational temperature compatible with our range of low temperatures (Sect. 4). The HST image (Sahai et al. 2000) suggests that the compact $\mathrm{CO}$ emission comes from the bright equatorial regions of the nebula. Therefore, in this source it is justified to calculate mass, momentum and energy for the fast component using the disk model described in Sect. 4.1 (with $i=15^{\circ}$ ), together with the standard bipolar and spherical models. As we can see in Table 3, the results do not strongly depend on the used geometrical model. Note the particularly high ratio between the gas and radiation momenta. 
Finally we note that this source is one of those characterized by a particularly low ${ }^{12} \mathrm{CO} /{ }^{13} \mathrm{CO}$ line ratio. Even for the line wings and the $J=1-0$ transition, the line ratio is <3. Similar results (e.g. Bujarrabal et al. 1990) have been interpreted as showing the presence of a very low ${ }^{12} \mathrm{C} /{ }^{13} \mathrm{C}$ abundance ratio in some evolved nebulae. See Sahai et al. (2000) for more details.

\subsubsection{IRAS $17436+5003$}

This source shows line wings that are weak, but can be identified even in ${ }^{13} \mathrm{CO} J=1-0$; in any case we have checked that the parameters obtained from ${ }^{12} \mathrm{CO}$ and ${ }^{13} \mathrm{CO} J=1-0$ are quite similar. No extent in the $\mathrm{CO}$ lines was detected by Bujarrabal et al. (1992), using the 30-m telescope. The ${ }^{13} \mathrm{CO} J=2-1 / J=1-0$ line ratio indicates a rotational temperature compatible with our general assumptions. We will accordingly use the ${ }^{13} \mathrm{CO} J=1-0$ transition with our standard procedure; an expansion velocity of $11 \mathrm{~km} \mathrm{~s}^{-1}$ for the slow component is used. Note that the inclination is not known, so we take a bipolar outflow with $i=30^{\circ}$. The lower limit to the momentum due to this assumption is just a factor 2 lower than the values given in Table 3 .

\subsubsection{He 3-1475}

He $3-1475$ shows wide CO profiles, from which we deduce $V_{\text {sys }}=50 \mathrm{~km} \mathrm{~s}^{-1}$ LSR. We have chosen a composite model with a slow component, corresponding to the line core and an expansion velocity $\sim 7 \mathrm{~km} \mathrm{~s}^{-1}$, and a fast outflow, that is assumed to be bipolar in view of the spectacular elongated structure of the nebula (Borkowski et al. 1997). The identification of the line core in this source is not straightforward, but we note that variations of its expansion velocity affect only slightly the momentum and energy calculations of the fast outflow, due to the very large velocity and strong $\mathrm{CO}$ emission of the later. The wings are detected in all lines; note some galactic contamination in ${ }^{12} \mathrm{CO} J=1-0$. We performed small maps that indicate a negligible $\mathrm{CO}$ extent, compared with the telescope resolution. The rotational temperature suggested by the line ratios is compatible with our general assumptions. Therefore, we apply to this source our standard procedure, for both the line core and the wings.

This source shows a very low ${ }^{12} \mathrm{CO} /{ }^{13} \mathrm{CO}$ line ratio; even in the relatively weak line wings and the $J=1$ 0 transition, the line ratio is $\$ 3$, see Sect. 5.1.3. If we recalculate the mass, momentum and energy from the ${ }^{12} \mathrm{CO} J=1-0$ (assuming an abundance $X\left({ }^{12} \mathrm{CO}\right)=$ $2 \times 10^{-4}$ ) we find for all three parameters values about three times smaller than those given in Table 3 .

\subsection{6. $89 \mathrm{Her}$}

The difference between line core and wings in the $\mathrm{CO}$ lines from 89 Her is not clear. We adopt, somewhat arbitrarily, a slow component velocity $\sim 3 \mathrm{~km} \mathrm{~s}^{-1}$ (note that the line is anomalously narrow, as always found in low-mass PPNe, see Sect. 8.2). The systemic velocity is $-8 \mathrm{~km} \mathrm{~s}^{-1}$. The analysis is simplified by the fact that no sign of departure from spherical symmetry and radial expansion is found from the CO mapping of this source (Alcolea \& Bujarrabal 1995), so the model would be the same for the line core and wings. These authors also confirm the small extent of the source. The line ratios are consistent with the low rotational temperatures adopted in our standard procedure.

\subsubsection{AFGL 2343 and IRC +10420}

AFGL 2343 and IRC +10420 are yellow hypergiant stars surrounded by a very thick circumstellar envelope (see de Jager 1998 and Sect. 2). We have included these two stars in our sample because its evolution may be similar to that of PPNe (Sect. 2), but we must keep in mind that they are relatively different objects than the rest of the sample.

Both stars show probably spherical envelopes with radial expansion. This is deduced for IRC +10420 from observations in $\mathrm{OH}$ maser and $\mathrm{CO}$ and $\mathrm{SiO}$ thermal emissions (Nedoluha \& Bowers 1992; Neri et al. 1998; Castro-Carrizo et al. 2001). For AFGL 2343, there are high-resolution CO data (Neri et al., in preparation) and accurate NIR mapping (Hawkins et al. 1995). Our $\mathrm{CO}$ spectra are consistent with this interpretation, they show very wide profiles with no clear core+wing structure; the total widths suggest high expansion velocities of $33 \mathrm{~km} \mathrm{~s}^{-1}$ and $35 \mathrm{~km} \mathrm{~s}^{-1}$ respectively for AFGL 2343 and IRC +10420 . We adopted $V_{\text {sys }}=100 \mathrm{~km} \mathrm{~s}^{-1}$, for AFGL 2343, and $V_{\text {sys }}=78 \mathrm{~km} \mathrm{~s}^{-1}$, for IRC +10420 . Note the variations in the profile from line to line, suggesting opacity effects in, certainly, the ${ }^{12} \mathrm{CO} J=2-1$ transition and also, probably, in the $J=1-0$ one. Data from Neri et al. suggest a $\mathrm{CO}$ source size much smaller than our $J=1-0$ beam; only for IRC +10420 the convolved size could be slightly larger than the telescope beam, for this source we introduced a correction of a factor 1.3 to the deduced values to take into account the extent. The line ratios are consistent with the low $T_{\text {rot }}$ adopted here.

We accordingly apply our standard procedure to AFGL 2343 and IRC +10420 , assuming spherical symmetry and radial expansion for the whole CO emission. Note the very high mass of these envelopes, already suggested from previous data.

For AFGL 2343 we adopted the distance deduced from the Hipparcos paralax, which is uncertain but compatible with the high luminosity of hypergiants. Recently, Josselin \& Lèbre (2001) have argued that this object could be a "normal" PPN, at a much shorter distance and with a much lower luminosity. If this is the case, the values of the mass and momentum we derive will be also smaller (proportionally to $D^{2}$ ), but the ratio between the gas and luminosity momenta will remain the same as that given in Table 3, indeed comparable to those found in most PPNe. 


\subsubsection{IRAS $19500-1709$}

This source shows a clear separation between slow and fast outflow components. The small CO extent (Bujarrabal et al. 1992) and the line intensity ratios confirm the assumptions in our standard model. The systemic velocity is deduced from the spectra to be $25 \mathrm{~km} \mathrm{~s}^{-1}$, and the expansion velocity of the slow component is taken to be $10 \mathrm{~km} \mathrm{~s}^{-1}$. Since the inclination is not known, we will use $i=30^{\circ}$, so the lower limit to the momentum (energy) is 2 (4) times smaller than the value in the table. We have checked that the ${ }^{12} \mathrm{CO}$ line gives similar values for the dynamical parameters.

\subsubsection{CRL 2477}

We recall the uncertain post-AGB nature of this source (Sect. 2). We also note the galactic contamination close to $V_{\mathrm{LSR}}=0 \mathrm{~km} \mathrm{~s}^{-1}$, we have restored the profiles by interpolating adjacent channels. Due to this contamination and to the unknown inclination of the source, we have assumed a unique bipolar component with $i=30^{\circ}$; remember that in this case the lower limit to the momentum is 2 times smaller than the calculated value. The adopted systemic velocity is $4 \mathrm{~km} \mathrm{~s}^{-1}$. Neri et al. (1998) deduced a very small size for the $\mathrm{CO}$ cloud and the very low $J=2$ $1 / J=1-0$ intensity ratio confirms the usually assumed low $T_{\text {rot }}$.

\subsubsection{CRL 2688}

This well studied source shows profiles clearly composed of a core plus wide wings. The extent of the line core CO emission (at half maximum) is $\sim 10^{\prime \prime}$ (Yamamura et al. 1996; Cox et al. 2000), which is taken into account in our calculations. Multiple jets are found in this nebula, but the overall inclination is relatively well known, see Sahai et al. (1998). From the maps by Cox et al., it can be seen that most of the line-wing emission comes from an axial flow. Some high-velocity CO emission also comes from a peculiar extension in the equatorial plane. We estimate from those maps that the bipolar approximation may be wrong for just $\sim 1 / 4$ of the wing integrated intensity; the possible correction is in any case small, compared to uncertainties due to other reasons. We also note that, given the small inclination angle, the effects on the calculated values of the geometry are particularly important in this source. In order to get an idea of the uncertainties due to the complex geometry, we are also giving in Table 3 calculations following the spherical-isotropic approximation for the fast outflow, although our discussion will be based on the results obtained following our bipolar model.

We have adopted, from the shape of the line core, $V_{\text {sys }}=-35 \mathrm{~km} \mathrm{~s}^{-1}$ and an expansion velocity for the slow component equal to $15 \mathrm{~km} \mathrm{~s}^{-1}$. We have checked that the ${ }^{13} \mathrm{CO}$ line ratio is compatible with the assumed low temperatures and that both ${ }^{12} \mathrm{CO}$ and ${ }^{13} \mathrm{CO} J=1-0$ lines give similar results (within a factor 2 , being the mass from the
${ }^{12} \mathrm{CO}$ data somewhat smaller, as expected if some opacity effect is present).

\subsubsection{NGC 7027}

NGC 7027 is a young PN, with a hot central star that shows particularly intense and well studied CO emission (contrarily to other $\mathrm{PNe}$ ). The analysis of our data on NGC 7027 is particularly difficult because of the extent of the $\mathrm{CO}$ emission, that is known to arise from a structure about 20-25" wide (Bieging et al. 1991; Graham et al. 1993). Our maps are not good enough as to allow an accurate spatial integration of the brightness, so we must assume an intrinsic source size and multiply by the brightness observed in the central position. We deduce that the area of the convolved profile is twice that of our $J=1-0$ beam. Due to the uncertainty of this correction, it is difficult to check that the line ratios are compatible with the assumed rotational temperatures. We will use $T_{\text {rot }}=15 \mathrm{~K}$, but we mention that if we instead take $30 \mathrm{~K}$, as proposed by Jaminet et al. (1991) from multiline CO observations, the correction to our values of the mass, momentum and energy would just be of a factor 1.6.

The accurate mapping by Bieging et al. and Graham et al. suggests that the CO emission mainly comes from a radially expanding shell. We therefore apply a sphericalisotropic model, considering separately an inner spectral region defined by the velocity range $5-51 \mathrm{~km} \mathrm{~s}^{-1}$ (we have assumed $V_{\text {sys }}=26 \mathrm{~km} \mathrm{~s}^{-1}$ ) and the rest of the profile: the weak and very wide wings, see Fig. 12. These remarkable very wide wings have been detected for the first and its origin is unknown, the asymmetry in the profiles may be due to observational effects.

The ${ }^{12} \mathrm{CO} /{ }^{13} \mathrm{CO}$ line intensity ratio in NGC 7027 shows the opposite phenomenon than for other PPNe mentioned above. The line ratio is very high, compared with the usual observations in evolved objects. This result may be due to the presence of selective photodissociation by UV photons from the inner hot star (see Fong et al. 2001, where it is discussed the case of this and other PNe that have developed a massive PDR and also show very faint ${ }^{13} \mathrm{CO}$ lines). Under this interpretation, ${ }^{12} \mathrm{CO}$ would survive in a relatively thick layer, much thicker than that in which ${ }^{13} \mathrm{CO}$ is abundant. In view of this phenomenon, we think that it is more meaningful to use for our purposes the ${ }^{12} \mathrm{CO} J=1-0$ observations. Therefore, the values of the calculated parameters may be underestimated for this source due to opacity effects, but this underestimate is probably minor for the high-velocity component, in view of their very low brightness and the large extent of the source. For the sake of comparison, we also show the mass, momentum and energy derived from ${ }^{13} \mathrm{CO} J=1-0$ for the inner intense spectral feature (assuming our standard ${ }^{13} \mathrm{CO}$ abundance). These figures then represent the dynamical parameters for the layer that is rich in ${ }^{13} \mathrm{CO}$, probably much thinner than the ${ }^{12} \mathrm{CO}$-rich shell. 


\subsubsection{IRAS $22272+5435$}

IRAS $22272+5435$ is one of the few PPNe emitting in CO that show no sign of high-velocity line-wings down to a good limit, compared to the intensity of the main component, that appears very similar to the profiles observed from shells around AGB envelopes. We use $V_{\text {sys }}=$ $-28 \mathrm{~km} \mathrm{~s}^{-1}$. The image in ${ }^{12} \mathrm{CO} J=1-0$ is practically circular and extending $21^{\prime \prime}$ (Neri et al. 1998), i.e. comparable to our beam. Assuming that the brightness distribution is the same for ${ }^{12} \mathrm{CO}$ and ${ }^{13} \mathrm{CO} J=1-0$, we derive that a correction of a factor 1.9 must be applied in our analysis to account for the extent. The ${ }^{13} \mathrm{CO}$ line ratio suggests a low rotational temperature. So, our standard method is applied to a unique spherical component.

Since the CO emission is very similar to that of AGB envelopes, we apply the usual methods to calculate mass loss rates and circumstellar molecular mass in such objects. We will follow the method described by Loup et al. (1993), that takes into account the CO excitation and opacity (from model grids) and the theoretical photodissociation radius. We use an expansion velocity of $8 \mathrm{~km} \mathrm{~s}^{-1}$, a fractional ${ }^{12} \mathrm{CO}$ abundance $X\left({ }^{12} \mathrm{CO}\right)=6 \times 10^{-4}$ (typical of C-rich AGB envelopes), and $D=1.7 \mathrm{kpc}$ (Table 1 ). (Note that in this source the ${ }^{12} \mathrm{CO} /{ }^{13} \mathrm{CO} J=1-0$ line ratio is larger than 20, in spite of the signs of opacity effects in ${ }^{12} \mathrm{CO} J=1-0$, suggesting that in this source $X\left({ }^{12} \mathrm{CO}\right) / X\left({ }^{13} \mathrm{CO}\right)$ must be larger than the values we have adopted up to now.) The results are $\dot{M}=$ $1.1 \times 10^{-5} M_{\odot} \mathrm{yr}^{-1}$ and a photodissociation radius $R_{\mathrm{CO}}=$ $4 \times 10^{17} \mathrm{~cm}$, with an envelope formation time $\sim 1.6 \times 10^{4} \mathrm{yr}$. For the used expansion velocity, these figures give a total mass in the CO-rich envelope $\sim 0.18 M_{\odot}$, very similar to that deduced from ${ }^{13} \mathrm{CO} J=1-0$ and our standard method $\left(0.14 M_{\odot}\right)$. Both results as well as the deduced values for the other parameters are given in Table 3 .

Following the method depicted in Sect. 5.2, we have (crudely) estimated an upper limit for the momentum carried by the bipolar outflow (if it exists). We used our ${ }^{13} \mathrm{CO} J=1-0$ spectrum, assuming that the wings are not detected down to a level $\sim 5 \%$ of the peak intensity and adopting that the typical width for the detected line is $20 \mathrm{~km} \mathrm{~s}^{-1}$. See results in Table 3 .

\subsubsection{IRAS $23304+6147$ and IRAS $23321+6545$}

The existing information on these two sources is poor. They have been observed in the optical by Hrivnak et al. (1999) and Ueta et al. (2000), showing a subarcsec image. IRAS $23321+6545$ has been mapped in CO by Neri et al., its image being also very small. We have only observed them in ${ }^{12} \mathrm{CO} J=2-1$ and ${ }^{13} \mathrm{CO} J=1-0$ being the ${ }^{13} \mathrm{CO}$ emission very weak, undetected in IRAS $23304+6147$. In view of the lack of data on them, we will apply our standard procedure.
For IRAS $23304+6147$ we will use the ${ }^{12}$ CO line, so we must keep in mind that opacity effects may be present (see discussion in Sect. 5.2). However, the intensity contrast between the ${ }^{12} \mathrm{CO} J=2-1$ and the ${ }^{13} \mathrm{CO} J=1-0$ is very strong, larger than $\sim 80$ (the theoretical intensity ratio derived for optically thin emission and the other assumptions used here). Therefore, we believe that such opacity effects are not very important in this source and that the derived values from ${ }^{12} \mathrm{CO} J=2-1$ are not strong underestimates. The systemic velocity is $-16 \mathrm{~km} \mathrm{~s}^{-1}$. For the central part of the line we use a spherical-isotropic model with expansion velocity equal to $9 \mathrm{~km} \mathrm{~s}^{-1}$. For the line wings, we use our bipolar model with $i=90^{\circ}$, so the momentum and energy results are directly the minimum values (if only geometry effects are considered).

For IRAS $23321+6545$ we will use the ${ }^{13} \mathrm{CO} J=1-0$ line, with the systemic velocity and velocity range deduced from the ${ }^{12} \mathrm{CO}$ line, respectively $V_{\text {sys }}=-55 \mathrm{~km} \mathrm{~s}^{-1}$ and $V_{\text {sys }} \pm 20 \mathrm{~km} \mathrm{~s}^{-1}$. Note the galactic contamination, that does not affect the ${ }^{13} \mathrm{CO}$ nebular line. We suppose a unique component in the bipolar approximation with $i=30^{\circ}$. The bipolarity of the outflow is in this case also suggested by the HST images (Ueta et al. 2000). For this source the distance is not known, we will adopt $D=1 \mathrm{kpc}$; this value could be a lower limit in view of the low values found for the distance-dependent parameters $(M, P, L$, etc.), which could be underestimated (but not the comparison of $M$, $P$ and $E$ with the stellar luminosity).

\subsubsection{2-56}

This source has been recently mapped with the Plateau de Bure interferometer (paper in preparation), confirming the presence of a high-velocity outflow and an hourglasslike structure. Model fitting of the maps per velocity indicates an inclination with respect to the plane of the sky $i \sim 15^{\circ}$. Only a small fraction of the source (a couple of extreme axial knots) is found to be placed in the limit of the $J=1-0$ beam of the $30 \mathrm{~m}$ telescope. In order to estimate the effect of the source extent, we performed a small map, the resulting correction for the wing emission shown in Fig. 16 is not very large, $\sim 40 \%$. The low value of $i$ suggests that the dynamical parameters in this source can be overestimated in the central hourglass-like region, since the projection correction may be too large if a component of the expansion is perpendicular to the axis. We recall that the overestimate of the momentum from our model with respect to an isotropical expansion is of a factor 2 .

The wings are very weak in ${ }^{13} \mathrm{CO} J=1-0$, so we used in our calculations the ${ }^{12} \mathrm{CO} J=1-0$ line. We have checked that the calculated mass values for the line core are not smaller when using the ${ }^{12} \mathrm{CO}$ line instead of the ${ }^{13} \mathrm{CO}$ line, so opacity effects are negligible even for the central velocities. We assumed a systemic velocity of $-27 \mathrm{~km} \mathrm{~s}^{-1}$ (LSR) and an expansion velocity for the slow component equal to $14 \mathrm{~km} \mathrm{~s}^{-1}$. 


\subsection{Other sources detected in CO emission}

For the PPNe detected in CO by other authors, we have chosen the best published spectra, i.e. those with the highest $\mathrm{S} / \mathrm{N}$ ratio, preferably ${ }^{13} \mathrm{CO}$ and $J=1-0$ lines, and with information as complete as possible on the observation conditions. The lines are analyzed as explained before, taking into account the effects due to the different beam sizes and efficiencies. When possible, i.e. for a few well studied nebulae, we will directly use the published results on mass and kinematics. See parameters of these nebulae in Table 2 and the results of our calculations in Table 4.

Note that our results for this group of sources, including the absence of line wings, are often very uncertain because of the scarce data on $\mathrm{CO}$ and on other needed parameters. The ${ }^{12} \mathrm{CO} J=2-1$ line, used when it is the only available spectrum with high $\mathrm{S} / \mathrm{N}$ ratio, probably leads to an important underestimate of the derived mass, momentum and energy, because of probable high opacity and possible resolution of the spatial source extent. See the case of Roberts 22 in Sect. 5.2.5. When other lines are used, our method gives mass values comparable to other estimates, see general discussion in Sects. 4 and 5.1.12, 5.2.3, 5.2.15.

For these CO lines taken from the literature, we will mainly analyze the line wings and the associated fast outflows. Often the quality of the observations is not good enough to allow a study of the slow component, since the definition of the different spectral features needs highquality profiles and since the opacity effects for the line core are expected to be particularly important.

We also tried to estimate limits in the cases in which the line wings are not detected. The treatment of limits in our case is very difficult, since when the line wings are not detected we lack also for information on the velocity. All we can do, under these conditions, is to perform crude estimates, based on the "reasonable" assumption that the extent of each line wing should be comparable to the width of the detected profile. The typical LSR velocity of the wing would then be equal to the systemic velocity \pm this width. Given the uncertainties of the analysis, we will only estimate limits to the scalar momentum. We did not consider the nondetections when only the ${ }^{12} \mathrm{CO} J=2-1$ line is available, since then the accumulation of uncertainties yields useless data. These limits are given in Table 4 for the four sources for which the method can be applied.

Now we discuss the details of the analysis source per source.

\subsubsection{Red rectangle}

This source shows narrow lines with wings, that will be analyzed as coming from a bipolar outflow. But we must keep in mind that the kinematics of the Red Rectangle may be different from that usual in PPNe (Jura et al. 1995). We will use the ${ }^{12} \mathrm{CO} J=2-1$ line by Jura et al., the best $\mathrm{CO}$ spectrum published for this source. We derive $V_{\text {sys }}=0 \mathrm{~km} \mathrm{~s}^{-1}$, and that the line wings extend between -7.4 and $-2 \mathrm{~km} \mathrm{~s}^{-1}$ and between 2 and $7.3 \mathrm{~km} \mathrm{~s}^{-1}$ LSR.
The source is assumed to be small compared to the beam of the telescope (IRAM $30 \mathrm{~m}$ ), since mapping with this instrument scarcely detected any extent (unpublished observations). Other parameters used are in Table 2.

\subsubsection{IRAS $07134+1005$}

This source shows no wings in the CO lines (Bujarrabal et al. 1992; Knapp et al. 1998), down to a limit of about $1 / 10$ of the peak for the ${ }^{12} \mathrm{CO}$ lines. We estimated a limit to the momentum using the ${ }^{12} \mathrm{CO} J=1-0$ line by Bujarrabal et al., and the prescriptions explained in Sect. 5.2; we adopted a typical width for the detected spectrum $\sim 20 \mathrm{~km} \mathrm{~s}^{-1}$. The CO source is not significantly extended, so no correction for this is done. The rest of the parameters used in the analysis (Table 2) are relatively well known for this source.

\subsection{3. $\mathrm{OH} 231.8+4.2$}

This nebula has been studied by Sánchez Contreras et al. (1997) using a method almost identical to ours and accurate mapping. We will adopt the results given in that paper without any change. As discussed by Sánchez Contreras et al. (1997) and Alcolea et al. (2001), the nebular mass obtained in this way is compatible with the values got from other molecular lines and FIR data.

\subsubsection{Hen 3-401}

The CO spectra published by Bujarrabal \& Bachiller (1991) show a profile about $30-40 \mathrm{~km} \mathrm{~s}^{-1}$ wide with some indication of fast outflow emission. The analysis of these data is very uncertain due to the poor profiles. In particular, only the ${ }^{12} \mathrm{CO} J=2-1$ line shows some indication of its shape, so an underestimate of the derived parameters is expected (see Sect. 5.2). We will assume, perhaps arbitrarily, that the line core extends about $22 \mathrm{~km} \mathrm{~s}^{-1}$ around the systemic velocity, $V_{\text {sys }} \sim-30 \mathrm{~km} \mathrm{~s}^{-1}$ LSR. Following Bujarrabal and Bachiller, we will assume that the extent of the source is significantly smaller than the telescope resolution.

\subsubsection{Roberts 22}

Observations of ${ }^{12} \mathrm{CO} J=2-1$ by Bujarrabal \& Bachiller (1991) show a very broad profile, about $100 \mathrm{~km} \mathrm{~s}^{-1}$ wide. As for other sources with similar characteristics (Sect. 5.1), we take the whole spectra in our analysis, and note that very similar results are obtained if a slow component with expansion velocities of about $10 \mathrm{~km} \mathrm{~s}^{-1}$ is considered. Bujarrabal and Bachiller note that the source does not seem to be extended. The values obtained for the total mass (Table 4) are lower by about a factor 10 than the results by Bujarrabal and Bachiller, from fitting of the CO line by a model of AGB envelope emission, and by Sahai et al. (1999b), from analysis of the circumstellar 
Table 4. Calculations of the mass, momentum and kinetic energy for the sources observed in CO by other authors.

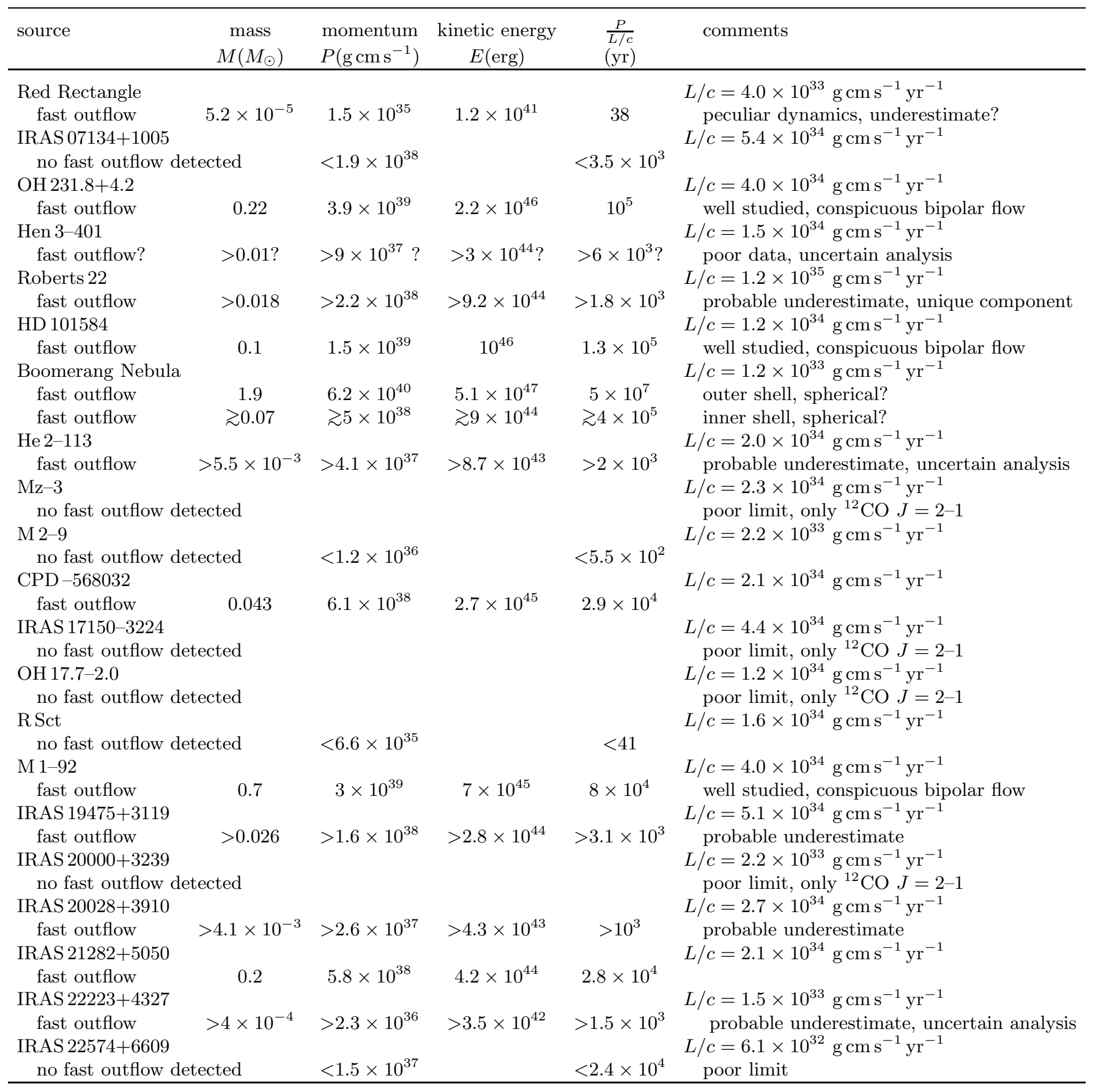

dust scattering. Probably the use of the usually opaque ${ }^{12} \mathrm{CO} J=2-1$ line in our analysis, that assumes optically thin emission, has led to a severe underestimate of the molecular mass and, therefore, of the outflow momentum and energy.

\subsubsection{HD 101584}

We will use the accurate observations by Olofsson \& Nyman (1999). These authors calculate mass and scalar momentum from their ${ }^{13} \mathrm{CO}$ and ${ }^{12} \mathrm{CO}$ spectra. We will adopt their values, correcting for an inclination $i=30^{\circ}$, as used here when the inclination is uncertain, and a ${ }^{13} \mathrm{CO}$ relative abundance equal to $2 \times 10^{-5}$, in order to be coherent with the rest of our paper. We also calculate the kinetic energy from their spectrum, using the above prescriptions.

\subsubsection{Boomerang Nebula}

The Boomerang Nebula was observed in ${ }^{12} \mathrm{CO} J=1-0$ and $J=2-1$ and ${ }^{13} \mathrm{CO} J=1-0$ by Sahai \& Nyman (1997). These authors found complex, very wide spectra that were interpreted as due to an outer very cold shell plus an 
inner less cold envelope. They fitted the data using detailed model calculations and assuming spherical shells with radial expansion at constant velocity. Even if this model is not similar to the bipolar outflows found in other $\mathrm{PPNe}$, we will take the mass of both components from that paper and calculate momentum and energy from the masses and velocities given there (Table 4). We note that, as we have discussed several times in this paper, the calculation of mass and momentum from the results by Sahai and Nyman probably depends only slightly on the assumed geometry and would be approximate in the case that one or both components are bipolar. Note that for the inner shell Sahai and Nyman only give a lower limit for the mass.

\subsubsection{He $2-113$}

We have used the ${ }^{12} \mathrm{CO} J=2-1$ profile published by Knapp et al. (1990), which shows wide line wings, although the profile structure is poorly defined. From discussion in Knapp et al. (1989), we infer that the units in Knapp et al. (1990) are equivalent to $T_{\mathrm{mb}}$. The CO extent is unknown, but optical and radiocontinuum images are very small, 1-2", see de Marco et al. (1997) and Bedding \& Zijlstra (1994), so we will assume that the CO shell is also much smaller than the telescope resolution in those observations $\left(32^{\prime \prime}\right)$. Since the inclination of the (putative) bipolar outflow is unknown, we will use $i=30^{\circ}$. Other parameters used in our analysis are $V_{\text {sys }}=-56 \mathrm{~km} \mathrm{~s}^{-1}$ (LSR) and $\mathrm{CO}$ line wings extending from -90 to $-67 \mathrm{~km} \mathrm{~s}^{-1}$ and from -48 to $-30 \mathrm{~km} \mathrm{~s}^{-1}$. In general, this object has not been well studied (not only in $\mathrm{CO}$ ), so the derived parameters remain quite uncertain.

\subsection{9. $\mathrm{Mz}-3$}

The ${ }^{12} \mathrm{CO} J=2-1$ observations by Bujarrabal \& Bachiller (1991) show a relatively narrow profile $\left(\sim 20 \mathrm{~km} \mathrm{~s}^{-1}\right.$ wide $)$ with no sign of wings. However the observational data are poor and the limit to the wing emission is just about $1 / 3$ of the line peak. No attempt to estimate limits is done for this source (see discussion in Sect. 5.2).

\subsubsection{2-9}

M 2-9 has been observed in ${ }^{12} \mathrm{CO} J=1-0$ and $J=2-$ 1 by Bachiller et al. $(1988,1990)$, with the $30 \mathrm{~m}$ IRAM telescope, and by Zweigle et al. (1997), who performed high-resolution mapping in ${ }^{12} \mathrm{CO} J=1-0$. Zweigle et al. found a small extent for the CO cloud, $\lesssim 10^{\prime \prime}$, as well as the absence of line wings down to a level of about $1 / 10$ of the peak, i.e. $T_{\mathrm{mb}}\left({ }^{12} \mathrm{CO} J=1-0\right) \lesssim 0.015 \mathrm{~K}$ (30 m-telescope scale; see also Bachiller et al. 1988). The velocity width of the lines in this source is very low, indicating an expansion velocity $\sim 7 \mathrm{~km} \mathrm{~s}^{-1}$. We will use these figures to crudely estimate a limit for the momentum in the fast outflow, if it exists, following the method in Sect. 5.2; no correction for the spatial extent was done.

Exceptionally, we will estimate the mass in the slow envelope in this source, since it is well mapped in CO and this value will be useful later (Sect. 8). Using our standard method and the above values, we get that the molecular mass in M 2-9 is $\gtrsim 6.1 \times 10^{-4} M_{\odot}$; the upper limit is taken following the discussion by Zweigle et al., who argue that the $\mathrm{CO}$ abundance in this source may be 10 times lower than the one adopted here. Indeed, the mass deduced from the fitting of the IR emission by Alcolea \& Bujarrabal (1991) is higher than $0.1 M_{\odot}$, and the extent of the beautiful nebula imaged in the optical is much larger than the $\mathrm{CO}$ ring; probably, $\mathrm{CO}$ has been strongly photodissociated in this source and its emission just probes a small fraction of the nebular material. Note that, corrected the abundance difference, the molecular mass value obtained here is in good agreement with that given by Zweigle et al.

\subsubsection{CPD -568032}

The most useful $\mathrm{CO}$ data for this source is the ${ }^{12} \mathrm{CO}$ $J=1-0$ spectrum by Nyman et al. (1992), see also Knapp et al. (1990). The profiles are clearly composite, with well defined line wings probably associated to a bipolar outflow. The CO extent is unknown but the optical image is very small, 1-2" (de Marco et al. 1997), so no correction for spatial extent is applied. The inclination of the source axis is unknown, therefore we take $i=30^{\circ}$. The derived velocities from the spectrum are $V_{\text {sys }}=-60 \mathrm{~km} \mathrm{~s}^{-1} \mathrm{LSR}$ and wings spanning from -130 to $-73 \mathrm{~km} \mathrm{~s}^{-1}$ and from -42 to $20 \mathrm{~km} \mathrm{~s}^{-1}$.

\subsubsection{IRAS $17150-3234$}

The best CO data are from Hu et al. (1993), who published a ${ }^{12} \mathrm{CO} J=2-1$ spectrum. The line is not well measured, showing a narrow profile (about $20 \mathrm{~km} \mathrm{~s}^{-1}$ ) and no sign of wings up to a limit of $1 / 5$ of the peak. No attempt to estimate limits to the outflow mass and momentum is done (see Sect. 5.2).

\subsubsection{3. $\mathrm{OH} 17.7-2.0$}

This source is difficult to observe in CO due to strong galactic contamination. The best published spectrum is that of ${ }^{12} \mathrm{CO} J=2-1$ by Heske et al. (1990), in which no line wing is detected, but to a poor limit (partially due to the galactic contamination). No attempt to estimate limits is done for this source (see discussion in Sect. 5.2).

\subsubsection{R Sct}

$\mathrm{R}$ Sct is a (peculiar) RV Tauri variable, then probably a post-red-giant object with low initial mass (e.g. Alcolea \& Bujarrabal 1991). R Sct is the only RV Tau star showing well detected $\mathrm{CO}$ emission. ${ }^{12} \mathrm{CO} J=2-1$ and $J=1-0$ 
and ${ }^{13} \mathrm{CO} J=2-1$ spectra of R Sct were published by Alcolea \& Bujarrabal (1991) and Bujarrabal et al. (1990). The lines are narrow $\left(\sim 10 \mathrm{~km} \mathrm{~s}^{-1}\right)$ with no wings down to a limit of about $1 / 10$ of the peak. We used their ${ }^{12} \mathrm{CO} J=$ 1-0 line to estimate a limit to the fast outflow momentum (Sect. 5.2); note the low value obtained (Table 4).

Also in this case, we will estimate the mass of the slow component from the ${ }^{12} \mathrm{CO} J=1-0$ data in Bujarrabal et al. (1990) and using our standard formulae. We obtain for the unique detected component a mass of about $8.3 \times 10^{-4} M_{\odot}$. This value is in good agreement with that derived by Alcolea \& Bujarrabal (1991) by fitting the photometric data on the SED of this source.

\subsubsection{1-92}

M 1-92, Minkowski's Footprint, is probably the best studied PPN in $\mathrm{CO}$ emission. We published accurate ${ }^{13} \mathrm{CO}$ $J=2-1$ maps (Bujarrabal 1998a), that were modeled using parameters (distance, inclination, ${ }^{13} \mathrm{CO}$ abundance, etc.) compatible with those given here. We therefore reproduce in Table 4 the results obtained in that paper for the well defined and studied bipolar outflow. We can see in Bujarrabal et al. (1998a,b) that the mass distribution obtained from this method is compatible with the data of the different CO lines and the results from observations of dust emission and scattering.

\subsubsection{IRAS $19475+3119$}

We take the ${ }^{12} \mathrm{CO} J=2-1$ spectrum by Likkel et al. (1991), since their $J=1-0$ data are poor. For these old data from the $30 \mathrm{~m}$ IRAM telescope, we will assume that the beam resolution is $\sim 14^{\prime \prime}$. We take $i=30^{\circ}$, in view of the absence of information on this parameter. We derive from the spectrum $V_{\text {sys }}=18 \mathrm{~km} \mathrm{~s}^{-1}$ LSR and the presence of wings from -10 to 8 and from 27 to $47 \mathrm{~km} \mathrm{~s}^{-1}$. The luminosity given in Table 2 was calculated by us, for the distance by Likkel et al. (1987), after integrating the IRAS fluxes, NIR fluxes from Hrivnak et al. (1994), and $V$ and $B$ magnitudes from the Simbad database.

\subsubsection{IRAS $20000+3239$}

${ }^{12} \mathrm{CO} J=2-1$ and $J=1-0$ were observed by Likkel et al. (1991), the $J=1-0$ line being only tentatively detected. Line widths $\sim 20-30 \mathrm{~km} \mathrm{~s}^{-1}$ were found. The profiles are apparently complex but do not show wings, with a limit to their intensity of about $1 / 5$ of the line peak. We could not find any indication on the distance to this source, so we assumed it to be $1 \mathrm{kpc}$ (probably an underestimate, see Sect. 5.1.13). No attempt to estimate limits is done for this source (see discussion in Sect. 5.2).

\subsubsection{IRAS $20028+3910$}

The ${ }^{12} \mathrm{CO} J=2-1$ profile by Likkel et al. (1991) shows a hint of line wings. We take $V_{\text {sys }}=6 \mathrm{~km} \mathrm{~s}^{-1}$ and wings between -17 and $-5 \mathrm{~km} \mathrm{~s}^{-1}$ and between 17 and $32 \mathrm{~km} \mathrm{~s}^{-1}$ (LSR). As in Sect. 5.2.16, we assume that the resolution of the 30 m IRAM's telescope in these observations was $\sim 14^{\prime \prime}$. The CO extent was tentatively detected by Neri et al. (1998), who give an ucertain value of the extent of $\sim 11^{\prime \prime}$ for ${ }^{12} \mathrm{CO} J=1-0$. HST images in the optical are about $2^{\prime \prime}$ wide (Hrivnak et al. 1999), and in the IR the source extent is about $1^{\prime \prime}$ (Meixner et al. 1999). We accordingly do not correct for the source extent in our calculations. The axis inclination is not known, so we take $i=30^{\circ}$.

\subsubsection{IRAS $21282+5050$}

This source has been relatively well studied in CO emission. Meixner et al. (1998) performed interferometric maps and found that $\mathrm{CO}$ is abundant in a (hollow) shell about $10^{\prime \prime}$ wide. However, line wings are detected only in the single-dish ${ }^{12} \mathrm{CO}$ data by Likkel et al. (1988). We will use their $J=1-0$ profile; no correction for the source extent is done here. We take $V_{\text {sys }}=18 \mathrm{~km} \mathrm{~s}^{-1}$ LSR and wings in the ranges $-4 \rightarrow 8 \mathrm{~km} \mathrm{~s}^{-1}$ and $30 \rightarrow 40 \mathrm{~km} \mathrm{~s}^{-1}$.

\subsubsection{IRAS $22223+4327$}

The best CO measurement in this source is the ${ }^{12} \mathrm{CO} J=$ 2-1 spectrum by Omont et al. (1993), see also Likkel et al. (1991). We assume in this case that the telescope beam is $14^{\prime \prime}$ wide at half maximum (Sect. 5.2.16). The CO extent is unknown in this source, but its IR image is very compact (1-2", Meixner et al. 1999), and we assume the CO source to be much smaller than the beam. We find from Omont et al's data, $V_{\text {sys }}=-30 \mathrm{~km} \mathrm{~s}^{-1}$ and wings between -52 and $-41 \mathrm{~km} \mathrm{~s}^{-1}$ and between -18 and $-8 \mathrm{~km} \mathrm{~s}^{-1}$. Due to the lack of data on this source, we assume $i=30^{\circ}$ and $D=1 \mathrm{kpc}$ (this value probably being a lower limit, see Sect. 5.1.13).

\subsubsection{IRAS $22574+6609$}

The best $\mathrm{CO}$ profiles of this source $\left({ }^{12} \mathrm{CO} J=2-1\right.$ and $J=1-0$, by Likkel et al. 1991) are $\sim 40 \mathrm{~km} \mathrm{~s}^{-1}$ wide and show no clear wings. The $\mathrm{S} / \mathrm{N}$ ratio and the profile shape in these observations are not good enough to allow a limit for the line wing emission better than about 1/10 of the peak. In the $J=1-0$ line one tentatively sees wings that are not confirmed in the $J=2-1$ profile. In any case, we used the $J=1-0$ line by Likkel et al. to crudely estimate a momentum limit for the fast outflow, with the method and limitations explained in Sect. 5.2. We assumed a typical width $\sim 20 \mathrm{~km} \mathrm{~s}^{-1}$ for the line core. Note that in this source the limit to the momentum is moreover uncertain due to the unknown distance, but that this does not affect the value of $\frac{P}{L / c}$, also quoted in Table 4 . 


\section{Characteristic duration of the strong mass acceleration}

As we will see in the next sections, we are interested in estimating, from the existing data, the time spent by the sources studied here as post-AGB stars $\left(t_{\mathrm{ppn}}\right)$ and the time during which the high-velocity $\mathrm{CO}$ outflows were accelerated $\left(t_{\text {acc }}\right)$.

An approximate upper limit to the value of $t_{\mathrm{ppn}}$ in individual sources is given by the typical value of the total post-AGB lifetime, 1000 yr. Such a value of the "transit" time from the AGB to the PN phase can be derived following several methods and agrees with theoretical estimates (see e.g. Bujarrabal et al. 1988).

When possible, $t_{\mathrm{ppn}}$ is calculated for individual sources from data on the $\mathrm{CO}$ outflow, i.e. dividing the characteristic length by the velocity, taking into account the inclination of the jets with respect to the plane of the sky (Table 1). When accurate CO data are not available, but the circumstellar shell is known to be detached and there are measurements of its inner radius $\left(r_{\text {id }}\right)$, we assume that $t_{\mathrm{ppn}} \sim r_{\mathrm{id}} / V_{\mathrm{agb}}$, where $V_{\text {agb }}$ is the expansion velocity of the AGB wind measured as the half-width of the CO line-core (see Sects. 4.1 and 8.1). A relatively uncertain estimate of $r_{\mathrm{id}}$ can be made from fitting the source's SED (spectral energy distribution) by means of dust absorption-emission models. In some cases, this estimate of $r_{\text {id }}$ is compatible with IR mapping (Meixner et al. 1997), but in general the results from pure SED fitting are uncertain (compare the measured values of $r_{\text {id }}$ in Table 5 for AFGL 2343, IRAS 22272+5435 and IRAS 07134+1005 with those estimated from the SED by Kwok et al. 1989; Hrivnak et al. 1989) and must be used with caution. In IRAS $21282+5050$ the inner radii determined from CO and IR mapping are significantly different; we suggest that this can be due to CO photodissociation by the stellar UV radiation, since this source is known to present a well developed central PDR with a mass $\sim 0.1 M_{\odot}$ (Fong et al. 2001). We will accordingly prefer the IR data in this case, but keeping in mind the uncertainty of the result. We will not use in our estimates the values of shell radii and velocities obtained from the HH-like objects emitting in atomic lines (like those observed in He 3-1475, Riera et al. 1995), since the interpretation in terms of $t_{\mathrm{ppn}}$ of the kinematics of this gas (probably ejected in the post-AGB phase and excited by counter-shocks) is not obvious. However, we will use the radii of the (empty) shells found by de Marco et al. (1997) from high-resolution mapping of $\mathrm{H} \beta$ emission, since those structures, when detected, are often comparable in size to the inner layers of the dense shells. The values we obtain for $t_{\mathrm{ppn}}$ are given in Table 5 . See velocities from the $\mathrm{CO}$ profiles in Sect. 5; some references on bipolar jets' structure are detailed in this section and those on the detached shell size are also given in Table 5. Other parameters needed in the calculation, like the distance and inclination, are taken from Table 1.

A first estimate of the typical value of the time needed to form the high-velocity flows (the acceleration time, $t_{\text {acc }}$ ) is given by statistical studies (Bujarrabal et al. 1992; Trammell et al. 1994), that show that bipolar ejections appear and are already massive in PPNe that are thought to be relatively young. Therefore, the strong gas acceleration took place at the beginning of its evolution and was very short, much shorter than the characteristic PPN lifetime. So, $t_{\text {acc }}<t_{\text {ppn }}$, for individual sources, $t_{\text {acc }} \ll 1000 \mathrm{yr}$, in general.

A better estimate of $t_{\text {acc }}$ can be reached in some cases, in which a clearly linear dependence of the velocity of the fast molecular gas on the distance to the central star has been found; such a result is sometimes called the "Hubble law" of the PPN kinematics. It has been argued (e.g. Alcolea et al. 2001 and references therein) that this law strongly suggests that the acceleration of the massive nebular flow was produced in a very short time, compared to the total lifetime of the nebula, since free movement is the easiest explanation of the Hubble law. The duration of such a process can readily be estimated from the dispersion of the empirical dependence of the velocity with the distance. The characteristic acceleration time $\left(t_{\text {acc }}\right)$ should be given by the characteristic PPN time (that can be derived with the same result for all points in which the law is satisfied), multiplied by a factor equal to the relative dispersion of the observational points in the velocity vs. distance diagram $(\Delta(r) / r)$. We must keep in mind that, since some part of this dispersion can be due to inhomogeneities in the density distribution or observational noise, the values so obtained could be overestimates of the actual $t_{\text {acc }}$. So, for these sources we will adopt $t_{\mathrm{acc}} \lesssim t_{\mathrm{ppn}} \times \Delta(r) / r$.

The typical acceleration times so calculated are astonishingly short. Mainly for the well studied cases, for which the above overestimate is not important. In OH231.8+4.2 (Alcolea et al. 2001), the "Hubble law" applies along a very elongated structure with a dispersion, $\Delta(r) / r \sim 1 / 5$; the kinematical PPN age of this nebula is $t_{\mathrm{ppn}} \sim 800 \mathrm{yr}$, so the acceleration time, $t_{\text {acc }}$, is $\$ 160$ yr. For M $1-92$ the velocity as a function of the distance was modelized by Bujarrabal et al. (1998a). From the comparison of the model predictions with the observations, we estimate that in this source $\Delta(r) / r \sim 1 / 8-1 / 10$; the PPN time $\sim 900 \mathrm{yr}$, so $t_{\text {acc }} \lesssim 100$ yr. The molecular outflow in HD 101584 also shows a clear distance dependence (Olofsson \& Nyman 1999), with a dispersion $\Delta(r) / r \sim 1 / 5$ and $t_{\mathrm{ppn}} \sim 150 \mathrm{yr}$; so, for HD 101584 we obtain $t_{\text {acc }} \lesssim 30$ yr. Note that we assumed in this calculation a standard value of the inclination of the flow axis with respect to the plane of the sky $\left(i=30^{\circ}\right.$ see Sect. 5.2.6), Olofsson and Nyman favor smaller values of this parameter, which would lead to even smaller acceleration times. Recent, unpublished CO observations of M 2-56 have also shown a linear distance dependence of the outflow velocity, with $\Delta(r) / r \sim$ $1 / 5$, and $t_{\mathrm{ppn}} \sim 1500 \mathrm{yr}$. These maps also show the presence of a central ring with an inner radius of about 1 $2^{\prime \prime}$, equivalent to $7 \times 10^{16} \mathrm{~cm}$; for an expansion velocity of the slow component of $14 \mathrm{~km} \mathrm{~s}^{-1}$, the obtained 
Table 5. Characteristic time spent by the studied sources as PPNe, $t_{\mathrm{ppn}}$, and time needed to accelerate the observed molecular outflows, $t_{\mathrm{acc}} . r_{\mathrm{id}}$ is the inner radius of the detached shell, when it can be estimated.

\begin{tabular}{|c|c|c|c|c|c|}
\hline name & $\begin{array}{c}r_{\mathrm{id}} \\
(\mathrm{cm})\end{array}$ & $\begin{array}{l}t_{\mathrm{ppn}} \\
(\mathrm{yr})\end{array}$ & $\begin{array}{l}t_{\mathrm{acc}} \\
(\mathrm{yr})\end{array}$ & refs. & comments \\
\hline IRAS $04296+3429$ & $10^{16}$ & 370 & $<370$ & 1,2 & \\
\hline CRL 618 & & 110 & $\leq 110$ & 0,3 & complex law for $V(r)$, well studied \\
\hline Frosty Leo & & $300-900$ & $\ll 500$ & 0,4 & \\
\hline IRAS $17436+5003$ & $\sim 1.1 \times 10^{17}$ & $\sim 2400$ & & 5 & uncertain $r_{\mathrm{id}}$ \\
\hline Нe 3-1475 & $?$ & $?$ & & & \\
\hline 89 Her & $?$ & $?$ & & & \\
\hline AFGL 2343 & $1.1 \times 10^{17}$ & 1100 & $\leq 1100 ?$ & 6 & yellow hypergiant \\
\hline IRC +10420 & $10^{17}$ & 900 & $\leq 900 ?$ & 7 & yellow hypergiant \\
\hline IRAS 19500-1709 & $4 \times 10^{15}$ & 120 & $<120$ & 5,2 & \\
\hline CRL 2477 & $?$ & $?$ & & & \\
\hline CRL 2688 & & 200 & $\leq 200$ & 0,8 & complex law for $V(r)$, well studied \\
\hline NGC 7027 & $8 \times 10^{16}$ & 1300 & $\overline{<} 1300$ & 9 & young PN, very fast outflow not considered \\
\hline IRAS $22272+5435$ & $1.5 \times 10^{16}$ & 480 & & 1,2 & no fast outflow detected \\
\hline IRAS $23304+6147$ & $\sim 2.9 \times 10^{15}$ & $\sim 100$ & & 1 & uncertain $r_{\mathrm{id}}$ \\
\hline IRAS $23321+6545$ & $?$ & $?$ & & & \\
\hline M 2-56 & $7 \times 10^{16}$ & 1500 & $\lesssim 300$ & 0 & well studied \\
\hline Red Rectangle & $\sim 1.5 \times 10^{15}$ & $\sim 120$ & & 10 & uncertain $r_{\mathrm{id}}$ \\
\hline IRAS $07134+1005$ & $4 \times 10^{16}$ & 1300 & & 2,5 & no fast outflow detected \\
\hline $\mathrm{OH} 231.8+4.2$ & & 800 & $\lesssim 160$ & 0,11 & well studied \\
\hline Roberts 22 & $?$ & $\lesssim 440$ & $<440$ & 12 & combining CO spectra and optical imaging \\
\hline HD 101584 & & 150 & $\lesssim 30$ & 0,13 & well studied \\
\hline He $2-113$ & $4.5 \times 10^{15}$ & 140 & $<140$ & 14 & \\
\hline M $2-9$ & $1.8 \times 10^{16}$ & 830 & & 15 & no fast outflow detected \\
\hline CPD -568032 & $6.8 \times 10^{15}$ & 140 & $<140$ & 14 & \\
\hline $\mathrm{OH} 17.7-2.0$ & $\sim 3 \times 10^{15}$ & $\sim 80$ & & 16 & uncertain $r_{\mathrm{id}}$, no fast outflow detected \\
\hline R Sct & $\sim 3 \times 10^{16}$ & $\sim 1900$ & & 10 & uncertain $r_{\mathrm{id}}$, no fast outflow detected \\
\hline M 1-92 & & 900 & $\lesssim 100$ & 0,17 & well studied \\
\hline IRAS $21282+5050$ & $5-10 \times 10^{16}$ & $1400 ?$ & $\leq 1400 ?$ & 18 & \\
\hline
\end{tabular}

References: (0): see text; (1): Kwok et al. (1989); (2): Meixner et al. (1997); (3): Neri et al. (1992); (4): Sahai et al. (2000); (5): Hrivnak et al. (1989); (6): Hawkins et al. (1995) (7): Castro-Carrizo et al. (2001); (8): Cox et al. (2000); (9): Graham et al. (1993); (10): Alcolea \& Bujarrabal (1991); (11): Alcolea et al. (2001); (12): Sahai et al. (1999c); (13): Olofsson \& Nyman (1999); (14): de Marco et al. (1997); (15): Zweigle et al. (1997); (16): van der Veen et al. (1995); (17): Bujarrabal et al. (1998); (18): Meixner et al. (1998).

kinematical post-AGB time is again $\sim 1500$ yr. Therefore, for M 2-56 we find $t_{\mathrm{acc}} \lesssim 300 \mathrm{yr}$.

In other less well studied cases, we only can assume that $t_{\mathrm{acc}} \leq t_{\mathrm{ppn}}$. Sometimes, the absence of shocks at present is complete, with no trace of even weak shocks, strongly suggesting that the interaction took place in a time much shorter than $t_{\mathrm{ppn}}$. This is the case of Frosty Leo (Sahai et al. 2000), in which the extent of the CO emitting region is $\sim 5^{\prime \prime}$ and the velocity dispersion observed is $\sim 80 \mathrm{~km} \mathrm{~s}^{-1}$. The kinematical age $t_{\mathrm{ppn}}$ ranges between $\sim 900 \mathrm{yr}$, calculated assuming isotropic expansion in the central region, and $300 \mathrm{yr}$ assuming bipolar ejections with an inclination with respect to the plane of the sky $i=15^{\circ}$ (Sect. 5.1.3). A different case is that of the fast outflow of CRL 618, which is small in the sky plane $\left(\sim 2^{\prime \prime}\right.$, see Neri et al. 1992) and show velocities projected in the line of sight $\sim 70-80 \mathrm{~km} \mathrm{~s}^{-1}$. In this source, the velocity/distance relation seems more complex than the linear dependence above discussed, and it is possible that some significant acceleration of the outflow is still taking place. For the inclination and distance values in Table 1, we calculate an age for the nebulae $t_{\mathrm{ppn}} \sim 110 \mathrm{yr}$. So the high momentum carried by the flow was adquired in a time $t_{\mathrm{acc}}$ comparable or smaller than this value. A similar calculation can be done for CRL 2688, from the maps by Cox et al. (2000), in which no "Hubble law" is found. Cox et al. calculated, using similar parameters than in our work and from the movements of the axial flow, values of $t_{\mathrm{ppn}}$ ranging between 250 and $125 \mathrm{yr}$, for all clumps except for the outermost northern component, for which $t_{\mathrm{ppn}} \sim 1200 \mathrm{yr}$. Since this outer clump just represents a very small part of the nebula, possibly decelerated by interaction with the slow wind, we can conclude that for CRL $2688 t_{\mathrm{acc}} \leq t_{\mathrm{ppn}}$ $\sim 200$ yr.

For the other sources of our sample, the poor maps or the lack of further information on the acceleration time lead to less meaningful limits, usually $t_{\mathrm{acc}}<1000 \mathrm{yr}$. 
Our best estimates (often limits) to the typical acceleration times of the fast outflows $\left(t_{\mathrm{acc}}\right)$ in individual objects are given in Table 5, together with our estimates of $t_{\mathrm{ppn}}$ and some comments on how they are determined. As we see, the typical values for $t_{\text {acc }}$ in protoplanetary nebulae are very low, particularly for the well studied sources. Although these estimates are crude, the results for the different sources are quite compatible, so they probably have, at least, a statistical meaning and can be used to derive a typical value $t_{\mathrm{acc}}=100-200 \mathrm{yr}$ for PPNe.

For the studied hypergiants, AFGL 2343 and IRC $+10420, t_{\mathrm{ppn}} \sim 1000 \mathrm{yr}$; these objects show clearly detached shells and $t_{\mathrm{ppn}}$ in them can be understood as the time elapsed since the end of the copious mass-loss process. $t_{\text {ppn }}$ may be an upper limit to the acceleration time also in hypergiants, if in these objects the high-mass shell was significantly accelerated after its ejection (as we are assuming for standard post-AGB objects). But the evolution of yellow hypergiants and the dynamics of their circumstellar shells are poorly known and we are not sure on the meaning of $t_{\text {acc }}$ in this case (see discussion in Sect. 5.1.7, particularly for AFGL 2343).

\section{Gas acceleration due to radiation pressure in late AGB stars and PPNe}

It is well known that the radiation pressure of the stellar light, acting primarily onto dust grains, can accelerate the circumstellar envelopes around AGB stars, explaining its expansion velocity $\left(V_{\mathrm{agb}}\right)$ and, in fact, its formation (e.g. Kwok 1975; Ivezić \& Elitzur 1995). In a first approximation, there is a limit for the momentum won by the outflow:

$\dot{M} \times V_{\text {agb }}<L / c$.

However, this is not exactly the momentum conservation law (that must be satisfied in any case), since we are dealing with "scalar momenta", i.e. with the momentum moduli integrated over a shell. When the dust opacity is very high, we must take into account the so called "multiple scattering": the fact that a stellar photon can be scattered, or absorbed and reemitted, several times before leaving the envelope. Under such a situation, the conservation law imposes (e.g. Knapp 1986; Ivezić \& Elitzur 1995):

$\dot{M} \times V_{\text {agb }}<\tau_{\text {eff }} \times L / c$.

Where $\tau_{\text {eff }}$ is the efficient opacity governing the multiple photon absorption. When the nebula is very opaque, which is our case, $\tau_{\text {eff }}$ is the opacity at the wavelength at which the maximum emission of the nebula takes place, 5-20 $\mu \mathrm{m}$, characteristic of the relatively cold dust grains. This is due to that once a photon is absorbed by the dust it is reemitted as radiation at the characteristic dust emission wavelengths, and continues being absorbed and emitted at these long waves.

The studies of the dust emission and absorption in AGB envelopes reveal that the most opaque shells (like that of IRC +10216 , with $\dot{M} \sim 2 \times 10^{-5} M_{\odot} \mathrm{yr}^{-1}$ ) have opacities in the optical and UV of about 10-20 (e.g. Rowan-Robinson \& Harris 1983; Rowan-Robinson 1980). We can assume, lacking for a better information, that when the late AGB star ejects its heavy envelope, with $\dot{M} \sim 1-2 \times 10^{-4} M_{\odot} \mathrm{yr}^{-1}$ (see Sect. 8.1), this dust opacity scales with the mass loss rate, reaching values $\sim 100$. In the IR, where $\tau_{\text {eff }}$ must be evaluated, we expect an opacity about 10 times smaller (for the usual dust extinction laws, see the above references), so for PPNe we can expect typical values $\tau_{\text {eff }} \sim 10$. Therefore, the ejection of this superwind must satisfy

$\dot{M} \times V_{\mathrm{agb}} \lesssim 10 \times L / c$.

Note that this formula gives a maximum momentum under the expected conditions, not an absolute maximum, since $\tau_{\text {eff }}$ is just a characteristic opacity.

\subsection{Efficiency of momentum transfer to the fast bipolar flows in PPNe}

Let us first introduce a general factor, $F_{\mathrm{ppn}}$, for estimating the maximum momentum won in this post-AGB acceleration during a characteristic time $t_{\text {acc }}$ :

$P \lesssim F_{\mathrm{ppn}} \times L / c \times t_{\mathrm{acc}}$

If we try to explain by radiation pressure the axial acceleration of the molecular nebula during the PPN phase, we must take into account that only about one half of the radiation momentum can be used to produce expansion along an axis. So, we conclude from the above discussion that $F_{\mathrm{ppn}}$ is typically $\sim 5-10$, when radiation pressure directly acts onto grains in the molecular flow. Of course, if we try to apply such a limit to the momenta obtained in Sect. 5, we must conclude that this process cannot explain at all the acceleration of the observed bipolar outflows.

Another argument against this simple mechanism has been found by Alcolea et al. (2001), based on that the very high drift velocity between the dust grains and the gas. Alcolea et al. argue that, if the outflow in the well studied PPN OH 231.8+4.2 is powered by radiation pressure acting primarily onto grains, one expects that the dust must flow at a velocity higher than that of the gas by $\sim 100 \mathrm{~km} \mathrm{~s}^{-1}$. This would produce structures in the images of dust scattered light and gas emission separated by a distance comparable to the size of the nebula itself. However, the observations indicate that both gas and dust features are exactly coincident, within the observational uncertainties: better than typically $5 \%$ of the distance to the center.

On the other hand, we have mentioned in Sect. 1 that there are good reasons to think that the momentum of the dense bipolar outflows is transferred to them from very fast and collimated post-AGB jets by shocks. Such jets (often observed to be expelled by the post-AGB stars, e.g. Kwok 2000) will be assumed to be completely different 
from the molecular flows (probed by CO lines), whose material was probably ejected by the AGB star. So the problem of the momentum in the PPN bipolar flows would be to explain the momentum of the post-AGB jets, particularly if we assume that they are powered by radiation pressure. But in this case we must recalculate the correction factor in Eq. (11). When the radiation pressure is transferred to the nebula by the intermediate of a shock front, a fraction of the energy of the impinging jet can be converted into scalar momentum. This holds when the shock is "energy driven", i.e. when the shock is adiabatic and the radiated energy is negligible. In this case, the temperature increases by a large factor in the shocked region where a kind of explosion is produced (in all directions). Since the ratio between the energy and momentum of the fast postAGB jets (i.e. its velocity) is much higher than for the dense molecular outflow, this conversion can significantly increase the "scalar momentum" won by the latter. This situation would not happen in the opposite case, when the shock is radiative (isothermal or momentum-driven shock), in which no significant correction factor appears.

The idea of that the shocks that accelerated the bipolar molecular outflow (observed in $\mathrm{CO}$ emission) could be strongly adiabatic is surprising. In many well studied cases, the properties of the accelerated material suggest the opposite. For instance, the temperatures of this accelerated gas are systematically found to be very low (10-20 K, see Sect. 4), indicating a very fast cooling after the passage of such an energetic shock. The very collimated molecular outflow found in $\mathrm{OH} 231.8+4.2$ suggests the presence of a "snow-plug" effect in the shock, which is characteristic of momentum-driven shocks and very different from the roughly isotropic inflation expected in adiabatic shocks. Sánchez Contreras et al. (2000) argue that, in view of the high densities calculated for this nebula (as for others), theoretical cooling models predict isothermal shocks in the inner parts of the nebulae, where the acceleration took place, and that adiabatic shocks would only appear in the very outer regions. The narrow walls of the fast-flowing cavities observed in several PPNe (M 192, Bujarrabal et al. 1998; Roberts 22, Sahai et al. 1999c; Frosty Leo, Sahai et al. 2000; etc.) also suggest a strong compression of the accelerated material, as expected in isothermal shocks. The case of Hen 3-401 is similar (Sahai et al. 1999b), in this nebula the very extended lobes show straight and narrow walls that had been predicted for momentum-driven shocks. However, the PPN Mz-3 and some PNe (e.g. Balick 2000), show inflated lobes that could be explained as the result of an adiabatic shock. Therefore, we cannot exclude the presence of adiabatic shocks in the early evolution of PPNe, but they are probably not a systematic phenomenon.

The increase of the momentum transferred to the dense shell due to the presence of adiabatic shocks is approximately limited by the ratio between the velocity of the fast post-AGB jets and the resulting velocity for the accelerated dense shell (neglecting the increase in internal energy). The velocity of the fast post-AGB jets responsible for the acceleration of the molecular flows is not known; hot, ionized gas is observed in $\mathrm{PNe}$ and $\mathrm{PPNe}$ with velocities as high as $1000-2000 \mathrm{~km} \mathrm{~s}^{-1}$ (e.g. Kwok 2000). Since the velocities of the molecular outflow usually range between 50 and $100 \mathrm{~km} \mathrm{~s}^{-1}$, we can give an estimate for the velocity ratio of approximately a factor 20 . This factor does not give the increase of the momentum in the axial direction due to adiabatic shocks, since in first approximation only the outwards axial movements would become the observed molecular outflows, a factor $1 / 4$ must then apply. Even if the collimation process is very efficient, it is difficult to accept that expansion in all direction becomes an outward, axial flow. Since we are looking for upper limits to the momentum transfer factor, we will assume that the resulting correction $F_{\mathrm{ppn}}$, to be included in Eq. (11), is then of about 10-20. We are aware that this estimate is uncertain, but our present knowledge does not allow us to think that $F_{\mathrm{ppn}}$ can be larger than this factor.

Note that if momentum is transferred to the fast molecular outflow by means of post-AGB jets, which are themselves accelerated by radiation pressure acting onto grains, we cannot assume that multiple scattering is significant. The reason is the very high velocity of the post-AGB jets, since the opacity in a flow varies inversely with the velocity for a given mass loss rate.

In summary, if radiation pressure acting onto grains is the final source of the momentum, we find that the maximum momentum won by the bipolar flows in PPNe must satisfy:

$P \lesssim F_{\mathrm{ppn}} \times L / c \times t_{\mathrm{acc}}$

If radiation pressure acts directly on the shell that will be accelerated, $F_{\mathrm{ppn}} \sim 5-10$. If radiation pressure acts by the intermediate of some very fast (and collimated) post-AGB wind, we get $F_{\mathrm{ppn}} \sim 10-20$. Since typically $t_{\mathrm{acc}} \sim 100$ 200 yr (Sect. 6), the limit to $P$ is:

$$
P \lesssim L / c\left(\mathrm{~g} \mathrm{~cm} \mathrm{~s}^{-1} \mathrm{yr}^{-1}\right) \times 2000(\mathrm{yr}) .
$$

The above limit is of course only approximate. We note that it has been derived assuming that the acceleration and properties of the late-AGB and post-AGB jets are similar to those presently observed in AGB stars. In particular, we have assumed that radiation pressure acts firstly onto grains. This is to be expected, since we have found that the acceleration is produced in the very first post-AGB phases, in which the star is still cool; see discussion on other scenarios in Sect. 9.

\section{Mass and dynamics of PPNe: Statistical results}

\subsection{The late $A G B$ wind}

We will assume that the slow component often observed in PPNe is the fraction of the fossil AGB envelope that has not been accelerated by the post-AGB wind interaction (see also Sect. 4.1). Firstly, its velocity is comparable to that commonly observed in AGB envelopes. Moreover, in several well mapped sources, the shape of 
this component is spherical and extended, as for AGB envelopes, suggesting that in them the axial disruption by wind interaction characteristic of PPNe has not reached the full extent of the AGB envelope. This is the case of CRL 618 (Yamamura et al. 1994), CRL 2688 (Yamamura et al. 1996) and IRAS 21282+5050 (Meixner et al. 1998). In the other well studied cases, this component is toroidal and perpendicular to the nebular symmetry axis, as expected if it is the residual of an extended axial acceleration; these are M 2-56, OH 231.8+4.2 (Alcolea et al. 2001), M 2-9 (Zweigle et al. 1997) and M 1-92 (Bujarrabal et al. 1998).

In order to estimate the mass loss rate responsible for the observed nebulae (that supposedly took place during the last phases of the AGB, and that was the responsible for its end), we have first estimated the outer radius of this slow remnant, $r_{\mathrm{os}}$. We recall that, in the well studied cases mentioned above, the extent of this CO shell is much smaller than that expected from photodissociation due to the interstellar UV field (see calculations by Mamon et al. 1988). So, the slow component is spatially bounded mainly by a sudden density decrease and not by molecule dissociation, and, therefore, its measured extent probably corresponds to the actual size of the shell formed under this regime of high mass ejection.

From $r_{\text {os }}$ and the slow component velocities, $V_{\text {agb }}$ (see Sects. 5, 6), we can calculate the time needed to eject the whole nebula by the former AGB star, $t_{\mathrm{s}}$. In this calculation, the inner radius $r_{\text {id }}$ is assumed to be very small, except for the hypergiant AFGL 2343, the young PN NGC 7027, M 2-56, M 2-9 and R Sct, for which we took values from Table 5 (Sect. 6). See values of $r_{\text {os }}$ and $t_{\mathrm{s}}$ in Table 6.

We can also estimate the total mass of the nebula, $M_{\text {tot }}$, from the masses of both (slow and fast) components detected in CO, see values in Table 6 . From $t_{\mathrm{s}}$ and $M_{\text {tot }}$, we estimate average mass loss rates during the late AGB phases. Only average rates are obtained, but since these values are probably the maximum allowed mass-loss rates (see below) it is possible that they were kept more or less constant during the relevant phase. The obtained values are listed in Table $6\left(<\dot{M}_{\text {lagb }}>\right)$, for the objects in which the existing data allow a reasonable estimate.

As we see in Table 6, the yellow hypergiants (AFGL 2343 and IRC +10420, see Sect. 2) show very massive molecular envelopes and very high mass-loss rates, between $5 \times 10^{-4}$ and $5 \times 10^{-3} M_{\odot} \mathrm{yr}^{-1}$. On the other hand, the PPNe with low initial mass ( 89 Her, M 2-9 and R Sct; no estimate was done for the Red Rectangle) show very low-mass molecular nebulae, as it was already known (e.g. Alcolea \& Bujarrabal 1991). Our analysis also gives a very high mass-loss rate (during its past AGB phase) for the young PN NGC 7027. This value is probably an upper limit, since the high velocity and the thin shell found in this source suggest that the bulk of the gas has been already compressed and accelerated by the passage of shocks and, therefore, our value $t_{\mathrm{s}}$ is probably an underestimate. The other, "normal" PPNe have total masses ranging in general between 0.1 and $1 M_{\odot}$. The only exceptions are IRAS 19500-1709 and IRAS 23304+6147; these also have very low luminosity, both low nebular mass and luminosity could be due either to that they are low-mass objects or (more probably) to an underestimate of the distance. The (late AGB) mass-loss rates that gave rise to these heavy envelopes range between $2 \times 10^{-5}$ and $3 \times 10^{-4} M_{\odot} \mathrm{yr}^{-1}$ for PPNe, with an average around $10^{-4} M_{\odot} \mathrm{yr}^{-1}$. We note that such large ejection rates are not observed in stars that are presently in the AGB, in which we hardly find rates larger than $2 \times 10^{-5} M_{\odot}$, the mass-loss rate of the famous, dense envelope of IRC +10216 (see e.g. Loup et al. 1993; Bujarrabal 1999; note that the total mass in the envelope around IRC +10216 is $\sim 0.2 M_{\odot}$ ).

Finally, we calculate the "scalar" momentum carried by this component per unit time when it was ejected, i.e. before the post-AGB wind interaction, $\dot{M}_{\text {lagb }} \times V_{\mathrm{agb}}$. (We recall that the scalar momentum is calculated by integrating the moduli of the momentum to the whole source, disregarding the vectorial nature of this parameter.) Its values are compared with the momentum carried by the stellar radiation, $L / c$. The resulting ratios are listed in Table 6 ; note that this parameter is distance independent. We find a very high momentum in the young PN NGC 7027; as we mentioned above, the whole nebula has probably won significant amounts of momentum in the post-AGB phase. The low-mass objects give relatively low values of the momentum. The rest of the nebulae, the standard PPNe and the hypergiants, give values of the ratio $\frac{\dot{M}_{\mathrm{lagb}} V_{\mathrm{agb}}}{L / \mathrm{c}}$ between 1 and 22 , with most objects presenting a value $\sim 5-10$. These figures are coincident with the expected maximum efficiency of momentum transfer for the stellar radiation pressure to gaseous shells, via photon absorption by dust grains (see Sect. 7).

The values of the mass-loss rate and the ejected mass during the last AGB phases are so large, compared to those commonly found in AGB stars, that the ejection of the planetary nebula and the end of the AGB phase should be interpreted as due to a discrete, quasi-explosive phenomenon of mass ejection, rather than to a smooth process lasting the whole AGB phase.

\subsection{Dynamics of the fast bipolar flows}

Only 4 sources in our sample (IRAS $22272+5435$, IRAS $07134+1005$, M 2-9 and R Sct), out of 32 for which reasonably good $\mathrm{CO}$ data exist, show no line wings down to a low level, i.e. less that $1 / 10$ of the peak. Of these sources, IRAS $22272+5435$ was accurately observed by us and shows no wings to less that $1 / 50$ of the peak in ${ }^{12} \mathrm{CO} J=2-1$. Five sources show no line wings, but the existing information is poor and the limits are not significant (Mz 3, IRAS 17150-3224, OH 17.7-2.0, IRAS 20000+3239 and IRAS 22574+6609).

As we have mentioned, the mass and momentum carried by the fast molecular flows in PPNe is very high. At least 9 PPNe in our sample have fast outflows with 
Table 6. Characteristic radius and formation time of the slow outflows in the PPNe that have been well observed in CO. We also quote the total nebular mass and the average mass rate at which the nebula was ejected during the late AGB phases.

\begin{tabular}{|c|c|c|c|c|c|c|c|}
\hline name & $\begin{array}{c}r_{\mathrm{os}} \\
(\mathrm{cm}) \\
\end{array}$ & $\begin{array}{c}t_{\mathrm{s}} \\
(\mathrm{yr}) \\
\end{array}$ & $\begin{array}{l}M_{\text {tot }} \\
\left(M_{\odot}\right) \\
\end{array}$ & $\begin{array}{l}<\dot{M}_{\text {lagb }}> \\
\left(M_{\odot} \mathrm{yr}^{-1}\right)\end{array}$ & refs. & $\frac{M_{\mathrm{lagb}} V_{\mathrm{agb}}}{L / c}$ & comments \\
\hline IRAS $04296+3429$ & $\sim 9 \times 10^{16}$ & $\sim 3000$ & 0.13 & $\sim 4 \times 10^{-5}$ & 0,1 & 3.1 & size from optical image \\
\hline CRL 618 & $1.5 \times 10^{17}$ & 2700 & 0.7 & $2.6 \times 10^{-4}$ & 0,2 & 7.6 & size from CO maps \\
\hline Frosty Leo & $\sim 2.3 \times 10^{17}$ & 7300 & 0.9 & $\sim 1.2 \times 10^{-4}$ & 0,3 & 22. & size from optical and CO images \\
\hline IRAS $17436+5003$ & $<2 \times 10^{17}$ & $<5000$ & 0.7 & $>1.4 \times 10^{-4}$ & 0 & $>1$ & assumed radius: $<3^{\prime \prime}$, from $\mathrm{CO}$ data \\
\hline Нe $3-1475$ & $\sim 1.5 \times 10^{17}$ & $\sim 7000$ & 0.63 & $\sim 9 \times 10^{-5}$ & 0,4 & $\sim 3.5$ & size from optical image \\
\hline 89 Her & $10^{16}$ & 1000 & $3.3 \times 10^{-3}$ & $4 \times 10^{-6}$ & 0,5 & 0.19 & size from CO mapping \\
\hline AFGL 2343 & $2.1 \times 10^{17}$ & 1000 & 4.8 & $4.8 \times 10^{-3}$ & 0,6 & 14. & $\mathrm{CO}+\mathrm{IR}$ size, unique velocity component \\
\hline $\mathrm{IRC}+10420$ & $4.5 \times 10^{17}$ & 4000 & 2.1 & $5.4 \times 10^{-4}$ & 0,7 & 1.4 & CO size, unique velocity component \\
\hline IRAS 19500-1709 & $<5 \times 10^{16}$ & $<1600$ & 0.033 & $>2 \times 10^{-5}$ & 0 & $>6.6$ & assumed radius: $<3^{\prime \prime}$, from $\mathrm{CO}$ data \\
\hline CRL 2477 & $5.9 \times 10^{16}$ & $?$ & $?$ & $?$ & 0,7 & $?$ & size from $\mathrm{CO}$, unidentified slow flow \\
\hline CRL 2688 & $1.8 \times 10^{17}$ & 3800 & 0.75 & $2 \times 10^{-4}$ & & 6. & size from CO mapping \\
\hline NGC 7027 & $1.8 \times 10^{17}$ & $>1500$ & 0.6 & $<4 \times 10^{-4}$ & 0,9 & $<43$ & size from $\mathrm{CO}$ mapping \\
\hline IRAS $22272+5435$ & $2.6 \times 10^{17}$ & 10000 & 0.2 & $2 \times 10^{-5}$ & 0,7 & 1. & size from CO mapping \\
\hline IRAS $23304+6147$ & $6 \times 10^{15} ?$ & $210 ?$ & $7 \times 10^{-3}$ & $3 \times 10^{-5} ?$ & 0,10 & $14 ?$ & poor information on extent \\
\hline IRAS $23321+6545$ & $4 \times 10^{16}$ & $?$ & $?$ & $?$ & 0,7 & $?$ & size from $\mathrm{CO}$, unidentified slow flow \\
\hline M 2-56 & $2.7 \times 10^{17}$ & 4500 & 0.11 & $2.3 \times 10^{-5}$ & 0 & 1.6 & size from CO mapping \\
\hline $\mathrm{OH} 231.8+4.2$ & $10^{17}$ & 4000 & 0.9 & $2.3 \times 10^{-4}$ & 11 & 9. & size from optical and CO images \\
\hline M 2-9 & $3.9 \times 10^{16}$ & 960 & $\gtrsim 6.1 \times 10^{-4}$ & $\gtrsim 6.410^{-7}$ & 0,12 & $\gtrsim 0.41$ & from CO mapping \\
\hline R Sct & $7 \times 10^{16}$ & 2500 & $8.3 \times 10^{-4}$ & $3.3 \times 10^{-7}$ & 0,13 & 0.02 & from CO mapping \\
\hline M 1-92 & $10^{17}$ & 4000 & 0.9 & $2.2 \times 10^{-4}$ & 14 & 9. & from $\mathrm{CO}$ mapping \\
\hline
\end{tabular}

References: (0): this paper; (1): Sahai et al. (1999a); (2): Yamamura et al. (1994), Meixner et al. (1998); (3): Sahai et al. (2000); (4): Borkowski et al. (1997); (5): Alcolea \& Bujarrabal (1995); (6): Hawkins et al. (1995), Neri et al., in preparation; (7): Neri et al. (1998); (8): Yamamura et al. (1996); (9): Graham et al. (1993); (10): Hrivnak et al. (1999); (11): Alcolea et al. (2001); (12): Zweigle et al. (1997); (13): Bujarrabal et al. (1990); (14): Bujarrabal et al. (1998).

mass $\gtrsim 0.1 M_{\odot}$. As we argue in Sect. 7.1 , the flow momentum $(P)$ cannot in principle be explained by radiation pressure when $\frac{P}{L / c}$ is larger than about $2000 \mathrm{yr}$. See values of $P$ and $\frac{P}{L / c}$ in Tables 3,4 . For our statistical study of the outflow momenta, we will not take into account the five objects in which the upper limits to the line-wing emission are not significant, one object in which the lower limit is too low to be useful (IRAS $20028+3910, \frac{P}{L / c}>1000 \mathrm{yr}$ ), two objects in which the comparison with radiation pressure momentum is not conclusive (IRAS 04296+3429: uncertain ratio $\frac{P}{L / c} \sim 1200 \mathrm{yr}$; IRAS 07134+1005: $\frac{P}{L / c}<$ $3500 \mathrm{yr}$ ), as well as the hypergiants (for which the typical times of the wind interaction are not well known). We are left with 27 objects that show reasonably useful CO data. Of them, only 6 nebulae (namely IRAS $22272+5435$, IRAS $23304+6147,89$ Her, the Red Rectangle, M 2-9 and R Sct), i.e. $22 \%$, show values of $\frac{P}{L / c}$ smaller than $2000 \mathrm{yr}$. Four of them are the four low-mass objects in our list (see further discussion below). For this $22 \%$ of the nebulae, the usual assumption that the stellar radiation pressure powers the bipolar protoplanetary flows is not incompatible with the observations. For the rest of the objects $(21$, $78 \%$ ), the momentum carried by the bipolar flows is too large to be explained, under standard conditions, by radiation pressure. Such a percentage is in any case a lower limit, since our calculation procedure can lead to underestimates of the mass and momentum in not well studied objects (Sect. 4.2), due to errors in the geometrical and $\mathrm{CO}$ emission models (significant overestimates are not expected). In particular, CO photodissociation by interstellar or stellar UV radiation could yield very low CO emission, despite a high actual mass of the envelope.

We could imagine that the correction factor $F_{\mathrm{ppn}}$ applied in Eq. (11) (Sect. 7) can be increased by some extra factor (of unknown origin). But there are PPNe in our sample with $\frac{P}{L / c} \gtrsim 10^{5} \mathrm{yr}$. These objects include precisely some of the best studied nebulae, for which the possible underestimate of the molecular masses inherent to our calculation method (Sect. 4.2) is expected to be less important: Frosty Leo, He 3-1475, OH 231.8+4.2, HD 101584, the Boomerang Nebula and M 1-92. A correction factor (Eq. (11)) $F_{\text {ppn }} \gtrsim 500-1000$ would be necessary. An inspection of the arguments in Sects. 7 and 9.1 shows that such high values of $F_{\text {ppn }}$ seems difficult to justify.

The case of the objects with low initial mass is remarkable: we have seen that the four studied nebulae (89 Her, the Red Rectangle, M 2-9 and R Sct; see Alcolea \& Bujarrabal 1991) are among the six sources showing comparatively low flow momentum. Only in the first two ones a relatively fast $\mathrm{CO}$ component is detected and with a mass not higher than $10^{-3} M_{\odot}$. It was known that these PPNe with low initial mass often show very weak and narrow CO lines. This may be due to a small CO abundance and/or extent in the nebula, probably because of 
photodissociation; this is particularly the case of $\mathrm{M} 2-$ 9 and of the Red Rectangle (Sect. 5.2.10, Alcolea \& Bujarrabal 1991). It is also possible that in low-mass objects the post-AGB evolution starts when only a small fraction of the stellar mass has been ejected, and so the momentum of the nebulae never reaches a high value. This could be the case of 89 Her and R Sct, where the mass derived from the FIR spectrum is low and comparable to that derived from $\mathrm{CO}$. But we note that the detection of the coldest grains from FIR dust emission is difficult, which would also yield an underestimate of the total nebular mass.

The hypergiants in our sample show very high nebular mass, $\gtrsim 2 M_{\odot}$, and momenta, with and $\frac{P}{L / c}$ equal to $1.2 \times 10^{4}$ (AFGL 2343) and $5 \times 10^{3}$ yr $($ IRC +10420$)$. Note also their high expansion velocities, higher than $30 \mathrm{~km} \mathrm{~s}^{-1}$. Since the acceleration times in them may be as high as 1000 yr (Sect. 2), we cannot exclude that the observed fast shells have been accelerated by radiation pressure with $\tau_{\text {eff }} \sim 10$; high values of this parameter can be expected in envelopes in which the mass-loss rate is as high as $10^{-3} M_{\odot} \mathrm{yr}^{-1}$. (It has been claimed that perhaps AFGL 2343 is not a hypergiant but a normal PPN, see Sect. 5.1.7, in this case this object would also have an overluminous fast outflow.)

The young PN NGC 7027 shows a main component with high velocity (larger than $20 \mathrm{~km} \mathrm{~s}^{-1}$ ) and mass $\left(\sim 0.5 M_{\odot}\right)$, that probably corresponds to most of the AGB envelope accelerated by post-AGB wind interaction.

We have also calculated the kinetic energy carried by the fast molecular outflows in PPNe (Tables 3, 4). Most of the standard PPNe show values of the kinetic energy in the range $10^{44}-10^{46} \mathrm{erg}$. Again low-mass post-AGB stars and hypergiants show values respectively under and over this range. We also note the very high energy carried by the Boomerang Nebula $\left(\sim 5 \times 10^{47} \mathrm{erg}\right)$, a peculiar PPN showing a very massive, fast and cold envelope (Sahai \& Nyman 1997). Note that the energy radiated by a postAGB star in 100-200 yr is of the order of $1-2 \times 10^{47} \mathrm{erg}$; so, from the point of view of the energy conservation, radiation pressure could explain the observed flows in PPNe with efficiency factors of a few per cent.

\section{Other mechanisms for the acceleration of the nebular material}

As we have seen (Sect. 8.2), radiation pressure under standard conditions (acting primarily onto grains) can explain the high mass-loss rates that, during the AGB phase, gave rise to the observed massive PPNe. However, this process seems unable to explain the great amounts of momentum won by a part of this envelope during the post-AGB phase, even if radiation pressure acts via post-AGB jets.

\subsection{Radiation pressure under unexpected conditions}

As we have mentioned in Sect. 8.2, the star radiates energy enough to explain the kinetic energies observed in the bipolar flows. The problem is the relatively low amount of radiated momentum. But the ill definition of our "scalar momentum" leads to some correction factors to the conservation law that are not easy to calculate.

We have argued that the presently observed post-AGB jets carry a relatively small mass and therefore the opacity of the dust in them should not be high (Sect. 7). We cannot discard however some unexpected situation in which the relevant opacity is not that of dust in the IR, like in the case of a transitional increase of the photospheric temperature allowing important absorption by lines or Ly $\alpha$ continuum, in which the efficient opacity could be much larger. We recall that this is not expected from what we know on this subject, since the bipolar flow acceleration seems to take place in the very first phases of the post-AGB evolution, when the star is still cool. For instance, only $\sim 10^{-9}$ of the total radiation of a $5600 \mathrm{~K}$ black body (corresponding to a G0-type star) takes place in the Ly $\alpha$ continuum. For these very low amounts of energy, what is relevant is not the momentum- but the energy-conservation law, that would avoid the acceleration of the flows by radiation pressure. In general we recall that stars with $L \sim 10^{4} L_{\odot}$ can typically radiate $2.5 \times 10^{47} \mathrm{erg}$ in $200 \mathrm{yr}$, though the energies of the flows are often $\sim 10^{46} \mathrm{erg}$. Perhaps a sudden reaccretion of previously ejected material could yield some kind of nova-like phenomenon in the stellar surface, leading to an important increase of the photospheric temperature (independently of an eventual increase of the luminosity). Perhaps a strong dependence of atmosphere's opacity on the stellar latitude would allow the presence of regions in which the radiation from the stellar very hot core can reach the surface (the poles?, due to centrifulgal distortion or non radial pulsations?), though the rest of the stellar surface still remains cool.

We could also imagine that the post-AGB jets are not produced under the form of a steady mass loss (as usually assumed in wind interaction models), but under the form of a sudden ejection of a large fraction of the stellar mass, in which a massive formation of dust occurs yielding a very large opacity. For instance, the sudden ejection of $0.1 M_{\odot}$, forming grains at about $5 \times 10^{14} \mathrm{~cm}$, could attain opacities $\sim 1000$ in the IR. But when this shell is accelerated, its opacity rapidly decreases and $\tau_{\text {eff }} \sim 1$ after only $\sim 30$ yr expanding at $200 \mathrm{~km} \mathrm{~s}^{-1}$; so in this case the acceleration time is extremely short.

We can still speculate on a different scenario, in which an exceptionally fast jet, with velocities $\gg 1000 \mathrm{~km} \mathrm{~s}^{-1}$, shocks with the AGB envelope at two points in the axis, producing a very hot adiabatic shock. By some mechanism, the resulting expansion would have been strongly collimated to yield the present axial flows. It is not clear how radiation pressure can yield so high velocities, since existing models predict moderate kinematics (Kwok 1975; Ivezić \& Elitzur 1995; etc.). (Note that, since these jets are produced in the very first post-AGB phases, the stellar properties are thought to be similar to those of AGB stars, for which those models are developed.) A theory explaining such a strong acceleration of the post-AGB jets 
by radiation pressure and the subsequent very energetic shocks, in particular why the dense accelerated gas does not radiate the internal energy (Sect. 7.1), is obviously missing.

\subsection{Other sources of energy}

When trying to find other sources of energy that could explain the observed outflows, the conservation of energy becomes the relevant constraint.

We can imagine that the energy needed could come from the ejection of the pulsating stellar layers after an ultimate pulse. However, the kinetic energy of $0.2 M_{\odot}$ pulsating at $20 \mathrm{~km} \mathrm{~s}^{-1}$ (a typical pulsation velocity) is just $\sim 8 \times 10^{44} \mathrm{erg}$. Also we note that we do not expect this pulsation to occur in only one direction.

Accretion onto the stellar surface may provide the energy required: $0.2 M_{\odot}$ falling in a star with a mass equal to $1 M_{\odot}$ and a radius equal to $10^{13} \mathrm{~cm}$ releases a gravitational energy $\sim 5 \times 10^{45} \mathrm{erg}$, compatible with the observed ones in most cases. This energy could be converted into kinetic energy in the axial direction by a process similar to that at work in forming stars and in active galactic nuclei, that involves the presence of magnetic fields and also explains the collimation of the axial jets. Theoretical studies for the specific case of PPNe would also be needed. The release of gravitational energy could be due to the approximation of a stellar companion, but then both stars would practically get in contact (with other cataclysmic consequences not observed). Moreover, it is difficult to understand why this approximation takes place, for almost all possible star pairs, exactly at the end of the AGB phase of the primary. Finally we note that the presence of a stellar companyon should help to produce accretion of circumstellar material. However, we still lack for a study quantitatively comparing the apparent ubiquity of the overluminous outflows in PPNe with the expected number of post-AGB stars belonging to the appropiate multiple systems; it should be also understood why, under the binary star assumption, such jets only appear in the AGB phase for a few well known symbiotic stars.

\section{Conclusions}

1: We present and analyze CO data on 37 objects, most of them protoplanetary nebulae (PPNe), including also the two hypergiants surrounded by heavy circumstellar envelopes, AFGL 2343 and IRC +10420 (that are probably following an evolution similar to that of PPNe), and the young planetary nebula NGC 7027 (that shows a some nebular properties similar to those of $\mathrm{PPNe}$ ). We think that our sample includes all well identified PPNe that have been detected in $\mathrm{CO}$. We have performed new very sensitive observations in 16 of these sources of ${ }^{12} \mathrm{CO}$ and ${ }^{13} \mathrm{CO}$ lines (both $J=1-0$ and $J=2-1$ transitions in most sources). For the others, we take $\mathrm{CO}$ data from the literature; in some of these sources, the published observations are found to be of poor quality for our purposes.
2: In the CO spectra, it is often possible to identify the different components characteristic of the $\mathrm{CO}$ emission from PPNe, namely the central core, corresponding to slow ejections (probably a remnant of the previous AGB wind), and the line wings, corresponding to bipolar fast flows (probably the result of the acceleration of a part of the AGB envelope by interaction with post-AGB fast, axial jets). The main goal of our study is the calculation of the mass, the linear momentum and the kinetic energy of these components from the $\mathrm{CO}$ spectra.

3: The method followed to calculate those parameters and the meaning and uncertainties of the derived results are discussed in detail. It is concluded that the errors are small (less than about a factor 2 in the developed examples), and that only significant underestimates of the mass and dynamical parameters can be expected. Errors are particularly small in well studied objects, in which the shell extent and the $\mathrm{CO}$ excitation and opacity can be determined.

4: The mass of the whole nebula is calculated, together with the associated mass-loss rates at which the total envelope was expelled during the late AGB phases (see Sect. 8.1, Table 6). The mass of most PPNe is larger than $0.1 M_{\odot}$, being often $\sim 1 M_{\odot}$. The corresponding mass-loss rates range between $2 \times 10^{-5}$ and $3 \times 10^{-4} M_{\odot} \mathrm{yr}^{-1}$, with typical values $\sim 10^{-4} M_{\odot} \mathrm{yr}^{-1}$. The exceptions are the objects with low initial mass (89 Her, M 2-9 and R Sct) and the yellow hypergiants (AFGL 2343 and IRC +10420 ), for which the the calculated masses and mass-loss rates are significantly lower and higher, respectively.

5: We also estimated the values of the "scalar" momentum (integration to the whole nebula of the momentum modulus) that was carried by this late AGB wind. After taking into account the effects of multiple scattering, we find the momentum of such a wind to be very close to the maximum momentum that can be supplied by radiation pressure. The duration of this high efficiency process is of a few thousand years, after which the star has lost a good fraction of its mass, its surface temperature starts increasing and the AGB phase ends.

6: We have studied the properties of the highvelocity molecular outflows by means of the line wings (often) detected in $\mathrm{CO}$ spectra. Only four objects (IRAS 22272+5435, IRAS 07134+1005, M 2-9 and R Sct) out of the 37 nebulae in our sample show no line wings to a low level (weaker than $\sim 1 / 10$ of the line maximum). Five others show no wings, but the existing observations are poor and do not allow meaningful conclusions.

7: Calculations of the momentum carried by the fast CO outflows yield very high values, between $10^{38}$ and $5 \times 10^{39} \mathrm{~g} \mathrm{~cm} \mathrm{~s}^{-1}$ in most PPNe. Their kinetic energy is also very high, often between $10^{45}$ and $10^{47} \mathrm{erg}$. The formation of these flows is the dominant phenomenon in the post-AGB dynamics. These values are compared with the momentum that can be provided by the stellar luminosity. We have taken into account the expected acceleration times, estimated for some sources, as well as correction factors due to eventual multiple scattering and 
conversion of energy into scalar momentum in adiabatic shocks (under the expected conditions). Of the 27 objects in which the observational data are good enough, only in 6 of them (IRAS $22272+5435$, IRAS $23304+6147,89$ Her, the Red Rectangle, M 2-9 and R Sct), $22 \%$ of our sample, the measured momentum could be explained by radiation pressure. In the rest of the nebulae (21 objects, $78 \%$ ), the stellar radiation is unable to explain the acceleration of the observed fast molecular flows. This percentage is probably a lower limit because, as we have mentioned, the calculated masses and momenta could be underestimates in not well studied sources.

8: In some cases, including most of the well studied objects, we find a ratio between the outflow momentum and the radiation momentum $\gtrsim 500-1000$. The correction factor, due to the effects mentioned above, can just reach a value $\sim 10-20$; values $\gtrsim 500-1000$ are completely unexpected for the standard conditions under which radiation pressure acts.

9: Among the six objects showing relatively low outflow momenta, we can find the four PPNe with low initial mass included in our sample (89 Her, the Red Rectangle, M 2-9 and R Sct). The weak CO emission from this kind of sources was already noticed. We discuss on the origin of this anomaly, that could be due to a low $\mathrm{CO}$ abundance or extent.

10: Excluding the four low-mass objects, in more than $90 \%$ of PPNe $(21 / 23)$ the fast bipolar flows would not be explained by radiation pressure. We conclude that the mechanism that powers the very energetic bipolar flows, and is finally reponsible for the PN shaping, is unknown, deserving theoretical developments.

11: We discuss different processes that could substitute the standard effect of radiation pressure. The most promising could be radiation pressure if unexpected opacity effects are present and conversion into bipolar jet momentum of gravitational energy of reaccreted material. Due to the lack of theoretical developments, this topic remains a matter of speculation.

Note added in proof: A very recent work, Zijlstra et al. (2001, MNRAS, 322, 280), has confirmed from $\mathrm{OH}$ maser data on several PPNe the general structure and velocity field used here, including the Hubble-like velocity law, which were deduced by us from $\mathrm{CO}$ observations.

Acknowledgements. This work has been financially supported by the Spanish DGES, under project PB96-0104. We are grateful to Noam Soker for fruitful discussions on the ejection mechanisms. We made use of the Simbad database, operated at CDS, Strasbourg, France.

\section{References}

Alcolea, J., \& Bujarrabal, V. 1991, A\&A, 245, 499

Alcolea, J., \& Bujarrabal, V. 1995, A\&A, 303, L21

Alcolea, J., Bujarrabal, V., Sánchez Contreras, C., Neri, R., \& Zweigle, J. 2001, A\&A, 373, 932

Allen, D. A. 1978, MNRAS, 184, 601
Allen, D. A., Hyland, A. R., \& Caswell, J. L. 1980, MNRAS, 192, 505

Andrei, A. H., Assafin, M., Puliaev, S. P., et al. 1999, AJ, 117, 483

Assafin, M., Andrei, A. H., Puliaev, S. P., et al. 1996, A\&AS, 117,335

Bachiller, R., Gómez-González, J., Bujarrabal, V., \& MartínPintado, J. 1988, A\&A, 196, L5

Bachiller, R., Martín-Pintado, J., \& Bujarrabal, V. 1999, A\&A, 227, 188

Balick, B. 2000, in Asymmetrical Planetary Nebulae II: From Origins to Microestructures, ed. J. H. Kastner, N. Soker, \& S. A. Rappaport, ASP Conf. Ser., 199, 41

Bedding, T. R., \& Zijlstra, A. A. 1994, A\&A, 283, 955

Bieging, J. H., Wilner, D., \& Thronson, H. A. Jr. 1991, ApJ, 379,271

Borkowski, K. J., Blondin, J. M., \& Harrington, J. P. 1997, ApJ, 482, L97

Bowers, P. F., Johnston, K. J., \& Spencer, J. H. 1983, ApJ, 274,733

Bujarrabal, V., Gómez-González, J., Bachiller, R., \& MartínPintado, J. 1988, A\&A, 204, 242

Bujarrabal, V., Alcolea, J., \& Bachiller, R. 1990, A\&A, 234, 355

Bujarrabal, V., \& Bachiller, R. 1991, A\&A, 242, 247

Bujarrabal, V., Alcolea, J., \& Planesas, P. 1992, A\&A, 257, 701

Bujarrabal, V., Fuente, A., \& Omont, A. 1994, A\&A, 285, 247

Bujarrabal, V., Alcolea, J., Neri, R., \& Grewing, M. 1997, A\&A, 320, 540

Bujarrabal, V., Alcolea, J., \& Neri, R. 1998a, ApJ, 504, 915

Bujarrabal, V., Alcolea, J., Sahai, R., Zamorano, J., \& Zijlstra, A. A. 1998b, A\&A, 331, 361

Bujarrabal, V. 1999, in Asymptotic Giant Branch Starts, IAU Symp. 191, ed. T. Le Bertre, A. Lébre, \& C. Waelkens, ASP, 363

Calvet, N., \& Cohen, M. 1978, MNRAS, 182, 687

Castro-Carrizo, A., Lucas, R., Bujarrabal, V., Colomer, F., \& Alcolea, J. 2001, A\&A, 368, L34

Cohen, M., Kunkel, W., Lasker, B. M., Osmer, P. S., \& Fitzgerald, M. P. 1978, ApJ, 221, 151

Cox, P., Lucas, R., Huggins, P. J., et al. 2000, A\&A, 353, L25

Crowther, P. A., de Marco, O., \& Barlow, M. J. 1998, MNRAS, 296, 367

Dayal, A., Hoffman, W. F., Bieging, J. H., et al. 1998, ApJ, 492, 603

Decin, L., van Winckel, H., Waelkens, C., \& Bakker, E. J. 1998, A\&A, 332, 928

de Jager, C. 1998, A\&AR, 8, 145

de Marco, O., Barlow, M. J., \& Storey, P. J. 1997, MNRAS, 292,86

de Marco, O., \& Crowther, P. A. 1998, MNRAS, 296, 419

Fong, D., Meixner, M., Castro-Carrizo, A., et al. 2001, A\&A, 367,652

Forveille, T., Morris, M., Omont, A., \& Likkel, L. 1987, A\&A, 176, L13

Goodrich, R. W. 1991, ApJ, 376, 654

Graham, J. R., Serabyn, E., Herbst, T. M., et al. 1993, AJ, 105,250

Groenewegen, M. A. T., Oudmaijer, R. D., Goudfrooij, P., van den Hoek, L. B., \& van Kerkwijk, M. H. 1996, A\&A, 305,475

Groenewegen, M. A. T., Whitelock, P. A., Smith, C. H., \& Kerschbaum, F. 1998, MNRAS, 293, 18 
Hawkins, G. W., Skinner, C. J., Meixner, M., et al. 1995, ApJ, 452,314

Heske, A., Habing, H. J., Forveille, T., Omont, A., \& van der Veen, W. E. C. J. 1990, A\&A, 239, 173

Hrivnak, B. J., Kwok, S., \& Volk, K. M. 1989, ApJ, 346, 265

Hrivnak, B. J., \& Kwok, S. 1991, ApJ, 368, 564

Hrivnak, B. J., Kwok, S., \& Geballe, T. R. 1994, ApJ, 420, 786

Hrivnak, B. J. 1995, ApJ, 438, 341

Hrivnak, B. J., Langill, P. P., Su, K. Y. L., \& Kwok, S. 1999, ApJ, 513, 421

Hu, J. Y., Slijkhuis, S., Nguyen-Q-Rieu, \& de Jong, T. 1993, A\&A, 273, 185

Ivezić, Z., \& Elitzur, M. 1995, ApJ, 445, 415

Jaminet, P. A., Danchi, W. C., Sutton, E. C., et al. 1991, ApJ, 380,461

Jones, T. J., Humphreys, R. M., Gehrz, R. D., et al. 1993, ApJ, 411,323

Josselin, E., \& Lèbre, A. 2001, A\&A, 367, 826

Jourdain de Muizon, M., Cox, P., \& Lequeux, J. 1990, A\&AS, 83,337

Jura, M., Balm, S. P., \& Kahane, C. 1995, ApJ, 453, 721

Kahane, C., Cernicharo, J., Gómez-González, J., \& Guélin, M. 1992, A\&A, 256, 235

Kastner, J. H., Weintraub, D. A., Zuckerman, B., et al. 1992, ApJ, 398, 552

Kelly, D. M., \& Latter, W. B. 1995, AJ, 109, 1320

Klochkova, V. G., Szczerba, R., Panchuk, V. E., \& Volk, K. 1999, A\&A, 345, 905

Knapp, G. R. 1986, ApJ, 311, 731

Knapp, G. R., Sutin, B. M., Phillips, T. G., et al. 1989, ApJ, 336,822

Knapp, G. R., Young, K., Lee, E., \& Jorissen, A. 1998, ApJS, 117, 209

Knapp, G. R., Gammie, C. F., Young, K., \& Phillips, T. G. 1990, in Submillimeter Astronomy, ed. G. Watt, \& A. Webster (Kluwer, Dordrecht), 33

Knapp, G. R., Bowers, P. F., Young, K., \& Phillips, T. G. 1995, ApJ, 455, 293

Kwok, S. 1975, ApJ, 198, 583

Kwok, S., Volk, K., \& Hrivnak, B. J. 1989, ApJ, 345, L51

Kwok, S. 1993, ARA\&A, 31, 63

Kwok, S., Hrivnak, B. J., \& Geballe, T. R. 1995, ApJ, 454, 394

Kwok, S., Hrivnak, B. J., Zhang, C. Y., \& Langill, P. L. 1996, ApJ, 472, 287

Kwok, S. 2000, The Origin and Evolution of Planetary Nebulae (Cambridge Univ. Press, Cambridge)

Le Bertre, T., Heydari-Malayeri, M., Epchtein, N., Gouiffes, C., \& Perrier, C. 1989, A\&A, 225, 417

Likkel, L., Morris, M., Omont, A., \& Forveille, T. 1987, A\&A, 173, L11

Likkel, L., Morris, M., Forveille, T., \& Omont, A. 1988, A\&A, 198, L1

Likkel, L., Forveille, T., Omont, A., \& Morris, M. 1991, A\&A, 246,153

Lopez, B., Mekarnia, D., \& Lefevre, J. 1995, A\&A, 296, 752

Loup, C., Forveille, T., Omont, A., \& Paul, J. F. 1993, A\&AS, 99, 291

Mamon, G. A., Glassgold, A. E., \& Huggins, P. J. 1988, ApJ, 328,797

Mauron, N., Le Borgne, J.-F., \& Picquette, M. 1989, A\&A, 218,213

Meaburn, J., \& Walsh, J. R. 1985, MNRAS, 215, 761

Meixner, M., Skinner, C. J., Graham, J. R., et al. 1997, ApJ, 482,897
Meixner, M., Campbell, M. T., Welch, W. J., \& Likkel, L. 1998, ApJ, 509, 392

Meixner, M., Ueta, T., Dayal, A., et al. 1999, ApJS, 122, 221

Nedoluha, G. E., \& Bowers, P. F. 1992, ApJ, 392, 249

Neri, R., García-Burillo, S., Guélin, M., et al. 1992, A\&A, 262, 544

Neri, R., Kahane, C., Lucas, R., Bujarrabal, V., \& Loup, C. 1998, A\&AS, 130, 1

Nyman, L.-Å., Booth, R. S., Carlström, U., et al. 1992, A\&AS, 93,121

Olofsson, H., \& Nyman, L.-A. 1999, A\&A, 347, 194

Omont, A., Loup, C., Forveille, T., et al. 1993, A\&A, 267, 515

Quinn, D. E., Moore, T. J. T., Smith, R. G., Smith, C. H., \& Fujiyoshi, T. 1996, MNRAS, 283, 1379

Reddy, B. E., \& Hrivnak, B. J. 1999, AJ, 117, 1834

Redman, M. P., O'Connor, J. A., Holloway, A. J., Bryce, M., \& Meaburn, J. 2000, MNRAS, 312, L23

Riera, A., García-Lario, P., Manchado, A., Pottasch, S. R., \& Raga, A. C. 1995, A\&A, 302, 137

Roddier, F., Roddier, C., Graves, J. E., \& Northcott, M. J. 1995, ApJ, 443, 249

Rowan-Robinson, M. 1980, ApJS, 44, 403

Rowan-Robinson, M., \& Harris, S. 1983, MNRAS, 202, 797

Sahai, R., \& Nyman, L.-A. 1997, ApJ, 487, L155

Sahai, R., Trauger, J. T., Watson, A. M., et al. 1998, ApJ, 493, 301

Sahai, R. 1999, ApJ, 524, L125

Sahai, R., Bujarrabal, V., Castro-Carrizo, A., \& Zijlstra, A. A. 2000, A\&A, 360, L9

Sahai, R., Bujarrabal, V., \& Zijlstra, A. 1999a, ApJ, 518, L115

Sahai, R., Zijlstra, A., Bujarrabal, V., \& te Lintel Hekkert, P. 1999b, AJ, 117, 1408

Sánchez Contreras, C., Bujarrabal, V., \& Alcolea, J. 1997, A\&A, 327, 689

Sánchez Contreras, C., Bujarrabal, V., Neri, R., \& Alcolea, J. 2000, A\&A, 357, 651

Schwartz, H. E., Aspin, C., Corradi, R. L. M., \& Reipurth, B. 1997, A\&A, 319, 267

Shenton, M., Monier, R., Evans, A., et al. 1994, A\&A, 287, 866

Skinner, C. J., Meixner, M., Barlow, M. J., et al. 1997, A\&A, 328,290

Szczerba, R., Omont, A., Volk, K., Cox, P., \& Kwok, S. 1997, A\&A, 317, 859

Swings, J. P., \& Andrillat, Y. 1979, A\&A, 74, 85

Trammell, S. R., Dinnerstein, H. L., \& Goodrich, R. W. 1994, AJ, 108, 984

Trams, N. R., Lamers, H. J. G. L. M., van der Veen, W. E. C. J., Waelkens, C., \& Waters, L. B. F. M. 1990, A\&A, 233, 153

Ueta, T., Meixner, M., \& Bobrowsky, M. 2000, ApJ, 528, 861

van der Veen, W. E. C. J., Habing, H.J., \& Geballe, T. R. 1989, A\&A, 226, 108

van der Veen, W. E. C. J., Omont, A., Habing, H. J., \& Matthews, H. E. 1995, A\&A, 295, 445

Volk, K., \& Kwok, S. 1989, ApJ, 342, 345

Volk, K., Kwok, S., \& Woodsworth, A. W. 1993, ApJ, 402, 292

Woodsworth, A. W., Kwok, S., \& Chan, S. J. 1990, A\&A, 228, 503

Yamamura, I., Shibata, K. M., Kasuga, T., \& Deguchi, S. 1994, ApJ, 427, 406

Yamamura, I., Onaka, T., Kamijo, F., Deguchi, S., \& Ukita, N. 1996, ApJ, 465, 926

Yuasa, M., Unno, W., \& Magono, S. 1999, PASJ, 51, 197

Zweigle, J., Neri, R., Bachiller, R., Bujarrabal, V., \& Grewing, M. 1997, A\&A, 324, 624 